\title{
WestVirginiaUniversity
}

THE RESEARCH REPOSITORY @ WVU

Graduate Theses, Dissertations, and Problem Reports

2008

\section{Cognitions of the community: The worldview of U.S. intelligence}

\author{
David Alex Mastro II \\ West Virginia University
}

Follow this and additional works at: https://researchrepository.wvu.edu/etd

\section{Recommended Citation}

Mastro, David Alex II, "Cognitions of the community: The worldview of U.S. intelligence" (2008). Graduate Theses, Dissertations, and Problem Reports. 4403.

https://researchrepository.wvu.edu/etd/4403

This Dissertation is protected by copyright and/or related rights. It has been brought to you by the The Research Repository @ WVU with permission from the rights-holder(s). You are free to use this Dissertation in any way that is permitted by the copyright and related rights legislation that applies to your use. For other uses you must obtain permission from the rights-holder(s) directly, unless additional rights are indicated by a Creative Commons license in the record and/ or on the work itself. This Dissertation has been accepted for inclusion in WVU Graduate Theses, Dissertations, and Problem Reports collection by an authorized administrator of The Research Repository @ WVU.

For more information, please contact researchrepository@mail.wvu.edu. 
Cognitions of the Community: The Worldview of U.S. Intelligence

David Alex Mastro II

Dissertation submitted to the Eberly College of Arts and Sciences at West Virginia University in partial fulfillment of the requirements

for the degree of

Doctor of Philosophy in

Political Science

R. Scott Crichlow, Ph.D, Chair

Kenneth R. Dombroski, Ph.D

Robert D. Duval, Ph.D

Joe D. Hagan, Ph.D

Susan Hunter, Ph.D

Department of Political Science

Morgantown, West Virginia 2008

Keywords: Political Psychology, Social Constructivism, U.S. Intelligence, World Politics Copyright 2008, David Alex Mastro II 


\section{ABSTRACT \\ Cognitions of the Community: The Worldview of U.S. Intelligence}

\section{David Alex Mastro II}

This dissertation attempts to determine what the worldview of U.S. intelligence looks like so that it can be incorporated into America's intelligence identity. I argue that this is necessary to gain a better understanding of why the agencies that comprise the U.S. Intelligence Community (IC) share several behaviors despite having different interests and preferences. To answer my research question, which asks what the worldview of U.S. intelligence is, I conceptualize the IC's core belief system about world politics (its worldview) as the five philosophical beliefs in an operational code. I hypothesize about how the IC views each of these cognitions in accordance with my theory that the IC's worldview underlies several U.S. intelligence norms. I also posit that the worldview of U.S. intelligence is specific and longstanding. However, after testing my hypotheses using automated content analysis and statistical methods, I only find strong support for two of my six propositions. The beliefs that comprise the IC's worldview are well-established and continuous, and U.S. intelligence believes that it has a low degree of control over historical development. My primary conclusion is that the IC's worldview is a topic that warrants further study given that cognitions underlie all political behavior and form the foundation for how power and interests are understood (Young and Schafer 1998, 84). I also conclude that the ultimate value of this dissertation lies not in what its analysis found, but in the fact that it is first study to empirically analyze the set of core beliefs that U.S. intelligence holds about foreign affairs and global issues among state and non-state actors. 


\section{ACKNOWLEDGEMENTS}

I want to thank my family and friends for supporting me during the time I spent researching and writing this dissertation. I also want to thank the members of my dissertation committee for their guidance, advice, and help in completing this study. 


\section{TABLE OF CONTENTS}

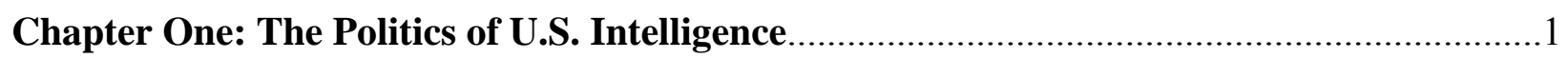

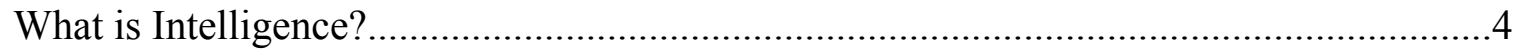

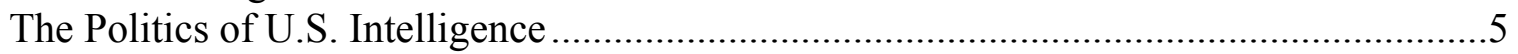

The Bureaucratic Politics Approach ……………………...................................................

The Social Constructivist Approach ………………...................................................... 10

The Need to Enrich the U.S. Intelligence Identity with Insights about Cognition .............16

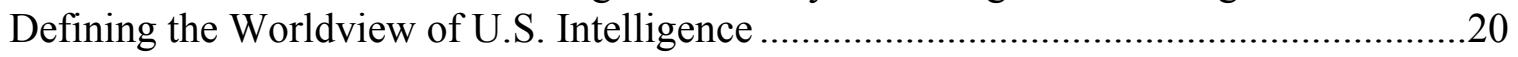

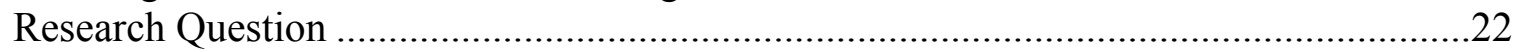

Chapter Summary and Dissertation Plan ......................................................................23

Chapter Two: Conceptualizing the Worldview of U.S. Intelligence ..................................26

Foreign Policy Analysis and Political Psychology ………….........................................22

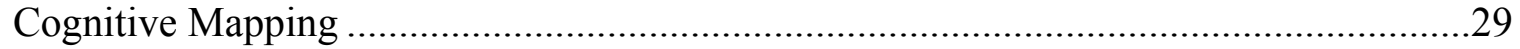

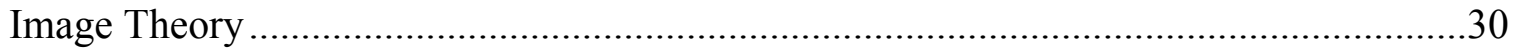

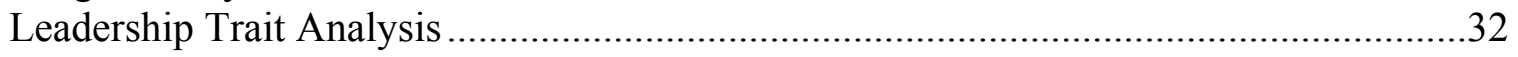

Operational Code Analysis .............................................................................................33

What is the Best Way to Conceptualize the Worldview of U.S. Intelligence?...................35

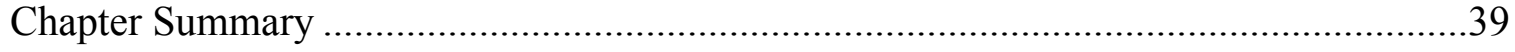

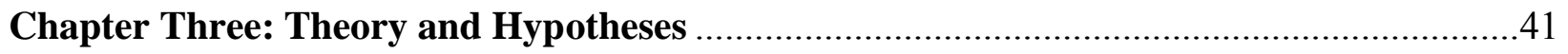

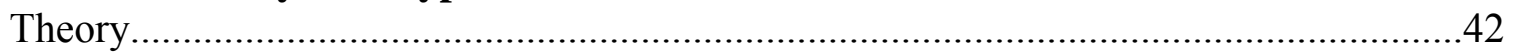

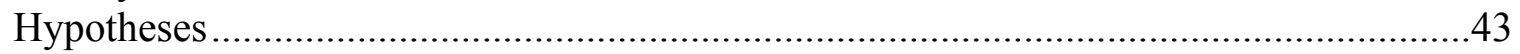

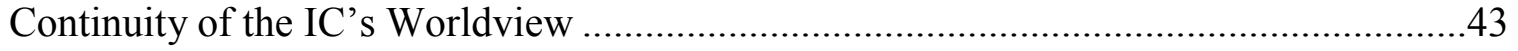

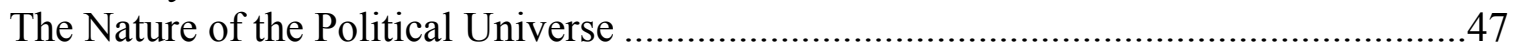

The Prospects for Realizing Fundamental Political Values...............................................50

The Predictability of Others in the Political Universe .........................................................53

Control Over Historical Development ............................................................................56

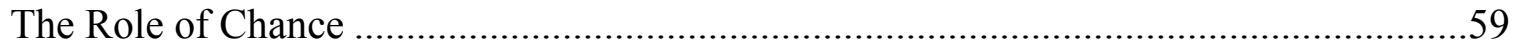

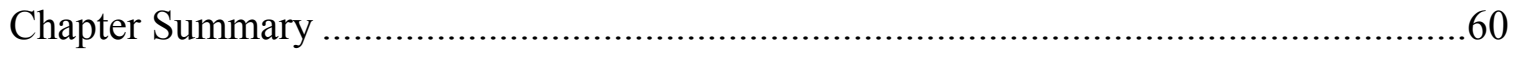

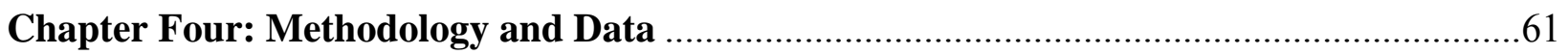

Quantifying the Worldview of U.S. Intelligence ...........................................................62

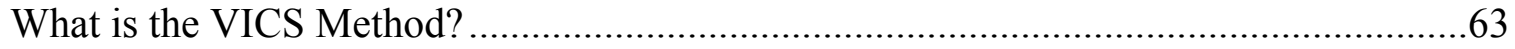

Calculating the Philosophical Indices ..............................................................................66

The Coding Approach: Computer Coding or Hand Coding? .............................................69

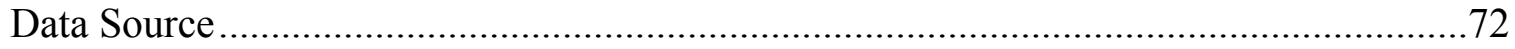

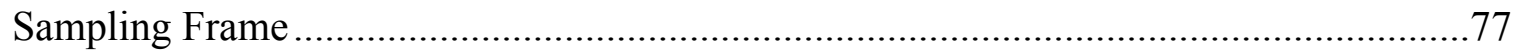

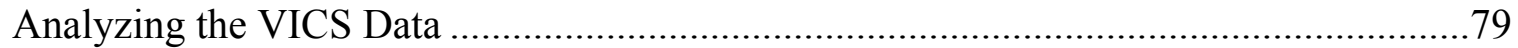

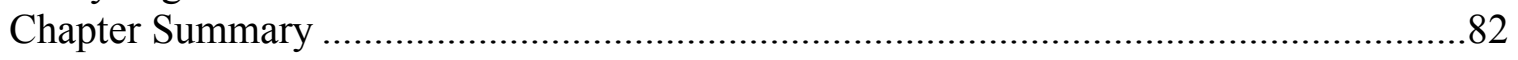




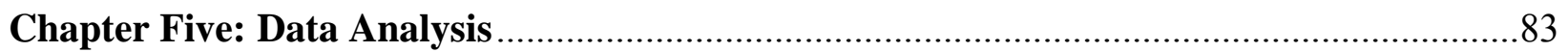

VICS Data on the Philosophical Beliefs in an Operational Code....................................84

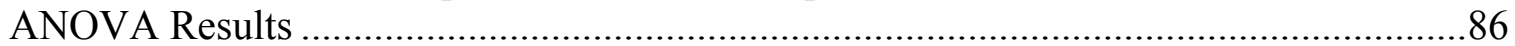

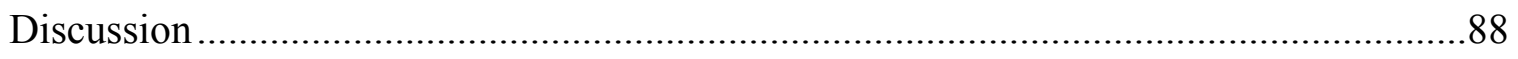

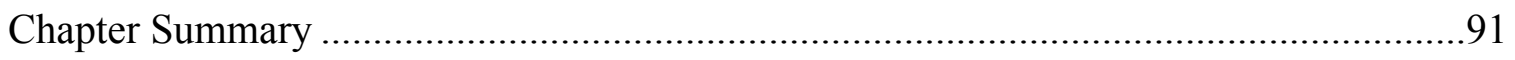

Chapter Six: General Conclusions: Future Research on the Cognitive Culture of..............92 U.S. Intelligence

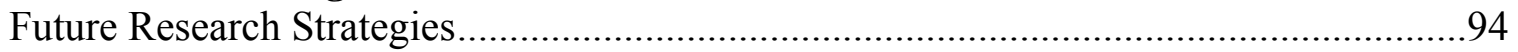

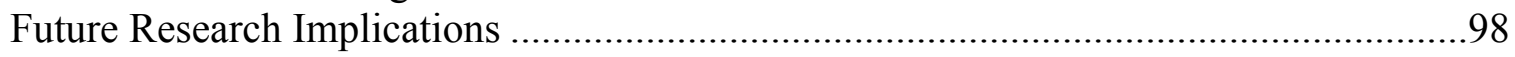

Chapter Summary and Concluding Remarks............................................................101

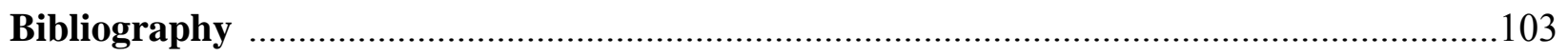

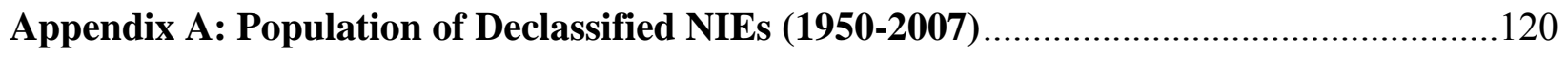

Appendix B: Sampling Frame of Declassified NIEs (1950-2007) ..................................122 


\section{LIST OF TABLES AND FIGURES}

Figure $1.1 \quad$ Levels of Organizational Culture................................................................ 19

Figure 4.1 Steps in the Verbs in Context System for Coding Verbs ..................................65

Figure 4.2 Calculating the Philosophical Indices.........................................................67

Figure 4.3 Balance Indices for the Five Philosophical Beliefs .......................................81

Table 5.1 Subgroup Scores and Mean Values for the Philosophical Indices ......................85

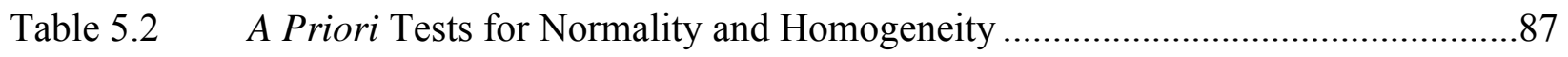

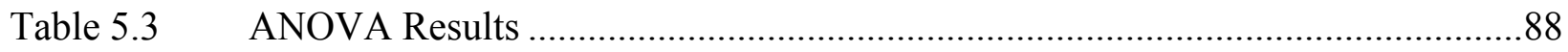




\section{CHAPTER ONE}

\section{THE POLITICS OF U.S. INTELLIGENCE}

Recent intelligence failures, such as the terrorist attacks of 11 September 2001 (9/11) and the "missing" weapons of mass destruction (WMD) in Iraq, have reminded Americans that good intelligence is crucial for national security (Sims and Gerber, eds. 2005, ix). ${ }^{1}$ As former President George H.W. Bush affirmed in a speech given in 1991, "intelligence remains our basic national instrument for anticipating danger, military, political, and economic. Intelligence is and always will be our first line of defense, enabling us to ward off emerging threats whenever possible before any damage is done" (Andrew 1995, 540). Consequently, it is not surprising that there are a substantial number of individuals and institutions within the United States (U.S.) government who require intelligence to perform their duties. This includes, but is not limited to, the president and his national security advisor; the vice president; the National Security Council (NSC) and its staff; the Attorney General; the Joint Chiefs of Staff (JCS); and the military services.

The organization charged with conducting intelligence activities for individuals and institutions within the U.S. government is the Intelligence Community (IC). The IC was formally established by the National Security Act of 1947, which President Harry Truman signed to realign and reorganize the U.S. armed forces, foreign policy, and intelligence apparatuses in the aftermath of World War II. Intelligence responsibilities were scattered among the various branches of the military prior to this time. This meant that the U.S. had no centralized

\footnotetext{
${ }^{1}$ Intelligence failures generally refer to when an event of strategic significance occurs without forewarning (Phythian 2006, 401). This focus is largely due to the fact that events of strategic significance generally pose the greatest threat to national interests. Moreover, relatively few intelligence failures are of consequence given that "most daily foreign policy decisions do not reflect the use of - or defense against — an application of power. Only when military, economic, or political power is actively applied do inadequacies in information become known" (Marrin 2004, 657).
} 
intelligence structure. ${ }^{2}$ The IC is currently led by the Director of National Intelligence (DNI) and consists of seventeen agencies or organizations. ${ }^{3}$ Its overarching mission is to carry out intelligence activities considered necessary for the conduct of foreign relations and the protection of U.S. national security. Thus, the primary areas of concern are the actions, policies, and capabilities of other nations and of other important non-state groups, such as international organizations and terrorist organizations (Lowenthal 2006, 5). ${ }^{4}$

Even though all U.S. intelligence agencies serve the common goal of informing or educating policymakers about threats to national security, the IC often functions in a way that promotes competition among the various agencies over information, money, people, and access (Turner 2006, 14). These agencies are bureaucracies seeking to increase their stature relative to others, promote their interests, and to survive in the political marketplace. During the process of trying to enhance their power and protect their turf, intelligence agencies often miscommunicate or do not communicate at all (Ibid., 14). This "noncooperation" is troublesome given that it is a leading cause of intelligence failure (Marrin 2006; Turner 1995, 2006). For example, the infamous antipathy between the CIA and the FBI contributed to some of the intelligence failures that led to $9 / 11$ (Hudson 2006, 77).

This paradox has led to a scant, but growing, literature on the politics of U.S. intelligence. ${ }^{5}$ This body of research tries to determine the origin of the intelligence agencies'

\footnotetext{
${ }^{2}$ See Aspin-Brown Commission (2004) for an historical overview of how the U.S. intelligence community evolved.

${ }^{3}$ These are the Office of the Director of National Intelligence (ODNI); the Central Intelligence Agency (CIA); the National Security Agency (NSA); the National Reconnaissance Office (NRO); the National Geospatial Intelligence Agency (NGIA); the Defense Intelligence Agency (DIA); the State Department's Bureau of Intelligence and Research (INR); the intelligence elements of the Air Force, Army, Navy, and Marine Corps; the counterintelligence component of the Federal Bureau of Investigation (FBI); the intelligence components of the Drug Enforcement Administration (DEA), the Department of Energy (DOE), the Department of the Treasury (DOT), the Department of Homeland Security (DHS), and the Coast Guard (CG).

${ }^{4}$ IC agencies also collect and produce intelligence on domestic security threats. The FBI is the IC's principle domestic intelligence agency.

${ }^{5}$ The literature on U.S. intelligence became a subject of serious academic study beginning in 1949 with the
} 
interests and preferences. To date, this literature has utilized organization theory (bureaucratic politics) and social constructivist theory to make its arguments. The former does an excellent job at explaining why IC agencies generally behave differently, whereas the latter helps explain why the intelligence agencies' also act similarly in several ways. However, social constructivists writing about intelligence have yet to fully explain the source of shared behaviors in the IC. ${ }^{6}$ They ignore the core beliefs that U.S. intelligence holds about world politics - its worldview. Incorporating the IC's worldview into the U.S. intelligence identity is imperative since it is partly responsible for shaping its identity. Its identity is also meant to help satisfy these beliefs.

The overarching goal of dissertation is to determine what the worldview of U.S. intelligence looks like. This is a necessary first step for incorporating the IC's worldview into its cultural identity since no study has yet to determine what beliefs comprise the IC's worldview. The remainder of this chapter clarifies the importance of, and the reasoning behind, taking a cognitive approach to better understand why IC agencies act similarly in various ways. It first defines the concept of intelligence for the purposes of this study. Next, it reviews the literature on the politics of U.S. intelligence. This leads to a critique of this literature. I demonstrate that the literature cannot fully explain the source of shared behaviors given that it ignores the IC's worldview. In the sections that follow, I define the worldview of U.S. intelligence and present this dissertation's research question, which asks what the IC's worldview is. The chapter concludes with a general summary and a discussion on how the remainder of this dissertation will be organized.

publication of Sherman Kent's Strategic Intelligence for American Foreign Policy.

${ }^{6}$ For instance, it cannot fully account for why the strict separation of intelligence from policy is almost a truism in the IC, why a top priority of all intelligence agencies is the protection of their sources and methods, and why all IC agencies put so much emphasis on the production of high-quality intelligence analysis. 


\section{What is Intelligence?}

Like the concept of terrorism, there is no agreed upon definition of intelligence (Warner 2002). Indeed, numerous studies grapple with the definition of intelligence (e.g., Betts 1978; Herman 1996; Hilsman 1958; Kent 1949; Laqueur 1985; Scott and Jackson 2004; Shulsky and Sims 1992; Troy 1991-1992). The term intelligence is often defined anew by each author who addresses it and these definitions rarely refer to one another or build off what has been written before. Notwithstanding, most definitions of intelligence begin with Sherman Kent's description of the concept (Johnson and Wirtz, eds. 2004, 2). Kent, who was an early theorist and practitioner of intelligence, defined intelligence as knowledge, as organization, and as an activity (Kent 1949, xi). This definition allowed him to describe the way intelligence services collect and analyze information, the finished intelligence product to policymakers, and the way intelligence services are organized.

The most succinct definition of intelligence based upon Kent's description of the concept is currently provided by Mark Lowenthal in his book Intelligence: From Secrets to Policy (2006). He describes intelligence as being "the process by which specific types of information important to national security are requested, collected, analyzed, and provided to policymakers; the products of that process; the safeguarding of these processes and this information by counterintelligence activities; and the carrying out of operations as requested by lawful authorities" (9). This definition does an excellent job at describing what intelligence is, why it is important, and how it works. Furthermore, this definition focuses on issues related to national security. This is important for the purposes of this dissertation since issues related to national security are the IC's primary concerns. ${ }^{7}$ Lowenthal's definition of the concept is also extremely

\footnotetext{
${ }^{7}$ Issues related to national security refer to "defense and foreign policy and certain aspects of homeland security" (Lowenthal 2006, 5),
} 
useful in that in prioritizes foreign intelligence over domestic intelligence (6). Collecting and producing foreign intelligence dominates the IC's agenda (Posner 2005). Recognizing this fact is vital since determining the IC's worldview requires me to focus on beliefs related to foreign intelligence activities. In conclusion, given its numerous advantages, this dissertation utilizes Lowenthal's definition of intelligence.

\section{The Politics of U.S. Intelligence}

Despite the fact that all of the agencies that make up the IC serve the common goal of informing or educating policymakers about threats to national security, intelligence scholars generally agree that "“the community" is more accurately viewed as a collection of competing bureaucratic elements factionalized by their rivalries, jealously, and competition for attention, dollars, and power." (Keagle, Tunstall, and Maurer, eds. 1985, 109). These agencies are bureaucracies seeking to increase their stature relative to others, promote their interests, and to survive in the political marketplace. In turn, scholars generally analyze the politics of U.S. intelligence in realist terms, focusing on its role within the government's national security framework and the interests and power relationships between and among the intelligence agencies (Turner 2004, 42-43).

\section{The Bureaucratic Politics Approach}

The impetus for a realist approach to understanding the politics of U.S. intelligence was the popularity of a strong research agenda during the 1960s and 1970s that examined the influence of organizational process and bureaucratic politics on foreign policy decision making. The "first wave" of this literature demonstrated how attempting to work with and through large organized governmental groups inhibits "rational" foreign policymaking (Art 1973, 468). Scholars within this wave posited that organizations and bureaucracies put their own survival at the top of their list of priorities (Huntington 1960; Hilsman 1967; Neustadt 1970; and Schilling, 
Hammard, and Snyder 1962). They will jealously guard and seek to increase their "turf" and strength, as well as preserve what they consider to be their "essence" or "mission." Large organizations also develop standard operating procedures (SOPs), which permit little flexibility or creativity. Ultimately, because there is little alternative to implementation of policy by the bureaucracy, these factors prevent the policy from being carried out exactly as its creators intended.

First wave theorists provided crucial insights into the way process affects the content of policy, but they did not tell us what characterizes this political process. A "second wave" of literature came about to remedy this (Art 1973, 472). The theorists of this wave tightened up the central propositions of the first wave to form a clearer picture of what the bureaucratic politics approach should look like. This began with Graham T. Allison's article “Conceptual Models and the Cuban Missile Crisis" (1969) and continued with his book The Essence of Decision (1971). In these works, Allison developed two new theoretical paradigms to demonstrate how bureaucratic politics affects foreign policy_an organizational process model and a governmental politics model. The former focuses on intraorganizational factors that affect bureaucratic behavior, whereas the latter emphasizes interorganizational factors. Allison eventually teamed up with Morton H. Halperin and wrote "Bureaucratic Politics: A Paradigm and Some Policy Implications" (1972). This piece combined the two models, which gave birth to the "bureaucratic politics" model.

Nonetheless, it was not until the publication of Morton H. Halperin's Bureaucratic Politics and Foreign Policy in 1974 that the bureaucratic politics model became fully formalized. This book examines several cases, in contrast to Allison's one, and offers “an extremely detailed amalgam of generalizations about bureaucratic behavior [within the U.S. government]" (Hudson 
2005, 8). This book contains the central propositions of the bureaucratic politics model. In short, per Halperin (1974), the bureaucratic politics model stresses the policymaking effects of interaction and competition among bureaucratic organizations and the competing roles of people within them. It posits that all bureaucratic organizations pursue their own purposes, promote their own power, attempt to enhance their own position in the governmental hierarchy, and strive to endure. The successful pursuit of these objectives flows from and reinforces internal SOPs and the way bureaucratic organizations mold the behavior of their role occupants. In the end, the bureaucracies are compartmentalized, resistant to change, competitive with one another, and intransigent toward presidential foreign policy initiatives. These cause foreign policy decisions to be the byproduct of bureaucratic in-fighting and compromise.

Besides the fact that Halperin's book tightened up the bureaucratic politics model, it also provided the first insights into the bureaucratic behaviors of IC agencies. A sizable portion of this book is dedicated to explaining how intelligence agencies, especially the CIA, attempt to influence foreign and national security policy. According to Halperin, "the only way members of the intelligence community can signal what policy they think should be adopted is by shaping the arguments about policy consequences to make clear what they think should be done" (137138). Halperin dissects the many strategies that intelligence agencies can use to manipulate decisions within the foreign policy bureaucracy to explain how this is done. He focuses on the degree to which participants plan their maneuvers, information and arguments, the process by which presidential decisions are made, and the sources of power in the bureaucracy and types of decisions that emerge. Halperin concludes that even though these strategies will affect the outcome of decisions that come to the President for approval, the President ultimately decides. Thus, intelligence agencies are limited in their ability to influence policy outcomes and realize 
their policy preferences.

Halperin's book also provides insight into why the interests and preferences of the IC agencies often diverge and, in turn, why the intelligence agencies' generally behave differently. Consensus is inhibited by the procedures through which IC agencies gather and report information, their need to protect long term interests, their need to defer to their own experts, and un-shared global images (139-155). Organizational or bureaucratic interests and preferences get in the way of inter-agency cooperation. These factors cause intelligence agencies to frequently disagree about intelligence interpretation, analysis, and conclusions. Therefore, the intelligence agencies' are likely to behave dissimilarly most, if not all, of the time.

The next major study on the bureaucratic behavior of U.S. intelligence agencies was The Fate of Facts in a World of Men: Foreign Policy and Intelligence-Making (1976) by Thomas L. Hughes. Hughes argues that while decision makers tend to hear what they want no matter what intelligence says, intelligence agencies tend to report what their leaders want to hear regardless of what the facts say. The behavior of IC agencies is conditioned by what their respective customers want to hear. For example, agencies such as the DIA will behave in accordance with the interests of the military, whereas organizations such as the CIA will act as advocates of the president and his advisors. ${ }^{8}$ The underlying cause of this is institutional survival. If an intelligence agency's customers deem its products to be irrelevant, it will be unable to survive in the political marketplace given that is will likely lose money, people, and access. This causes the intelligence agencies' to be competitive with one another and to disagree most of the time.

Several studies were undertaken to complement Hughes essay in the tradition of Halperin over the next two and a half decades. Seminal studies include Handel (1987), Hastedt (1985),

\footnotetext{
${ }^{8}$ The DIA is a major producer and manager of military intelligence for the U.S. Department of Defense, while the CIA is a civilian intelligence agency whose main function is to provide foreign intelligence to the President and his advisors.
} 
Jervis (1985), Lowenthal (1992), Picket (1985), Stempel (1999), Thomas (1985), Wirtz (1991), and Zegart (1999). These studies generally posit that bureaucratic politics does indeed determine the interests and preferences of U.S. intelligence agencies. This is evidenced by the fact that each agency has its own dedicated intelligence capability and each produces intelligence that it claims to be unique and relevant. Specialized collection mechanisms become the currency that intelligence agencies use to compete, outbid, or even undermine each other. During this process of trying to enhance their power and protect their turf, intelligence agencies frequently compete with one another and behave differently even though they all are charged with "[keeping] track of threats, forces, events, and developments that are capable of endangering the nation's existence" (Lowenthal 2006, 2).

The bureaucratic politics model explains much of the intelligence agencies' behavior. Indeed, "that bureaucratic politics exists is beyond question" (Rhodes 1994, 39). However, the bureaucratic politics model is by no means without its critics. One critique, originally offered by Stephen Krasner in 1972 about the bureaucratic politics model in general, suggests that this approach obscures the power of intelligence consumers, undermines democratic politics by relieving high officials of responsibility, and offers leaders an excuse for their intelligence failures (Cimbala 1988). Moreover, Jerel Rosati (1981) argues that "[t]he bureaucratic politics model does not apply to policymaking behavior as often as is popularly portrayed" (251).

The most vocal criticism from an intelligence scholar comes from Michael Turner (2004). He argues that even though the bureaucratic politics approach explains much of the intelligence agencies' behavior, it does not explain all of it. This is because this research agenda assumes that all of the intelligence agencies' interests and preferences are exogenously given. It expects the preferences of intelligence agencies to simply follow from their bureaucratic 
interests. By treating intelligence agencies as amorphous entities, which denies the sociopsychological nature of politics (Malici 2006, 127), scholars that analyze the politics of U.S. intelligence in realist terms are unable to explain the source of shared behaviors. ${ }^{9}$ Thus, they are unable to answer questions such as why much of the intelligence task takes place in secret; why the strict separation of intelligence from policy is almost a truism in the IC; or why U.S. intelligence agencies put so much emphasis on the production of high-quality intelligence analysis.

\section{The Social Constructivist Approach}

To move beyond the ontological reductionism of the bureaucratic politics approach, ${ }^{10} \mathrm{a}$ small group of intelligence scholars has emerged that utilizes the logic of social constructivism to delineate the orientations and preferences in the IC that would define its "identity" (Davies 2004; Sims 2005; Turner 1995, 2004, 2006; Wark 2003). Led by Jennifer Sims and Michael Turner, this group believes that

U.S. intelligence operates within a dominant strategic culture that, for better or worse, defines its myriad national security activities. Intelligence gets its cues from the strategic culture, producing a series of intelligence norms, or principles, that define collective expectations for the proper behavior of U.S. intelligence agencies in fulfilling their missions. The collection of norms, reinforced by a series of known rules and procedures, in turn forms the basis of a U.S. "intelligence identity" around which these expectations converge. (Turner 2004, 43)

These scholars ultimately posit that the U.S. intelligence identity guides the intelligence agencies' in their behavior given that the intelligence process "is conditioned by American culture and style, the organization of U.S. national security decision making, and the perception and proclivities of American leaders" (Cimbala 1988, 73).

\footnotetext{
${ }^{9}$ One exception to this could be when the interests and preferences of the intelligence agencies converge. However, this argument undermines the bureaucratic politics model since the model assumes that each intelligence agency has different interests and preferences due to different customers and specialized collection capabilities.

${ }^{10}$ Ontological reductionism is the idea that everything that exists is made from a small number of basic substances that behave in regular ways.
} 
Social constructivism became popular after the events surrounding the end of the Cold War cast serious doubt on the universal applicability of structural theories of international relations (IR) (Malici 2006, 129). ${ }^{11}$ Structural theories, like the bureaucratic politics model, assume exogenously defined preferences. This causes them to suffer from several shortcomings. They are unable to fully explain how preferences are formed, where they come from, or "how individuals under given conditions produce new conditions" (Przeworski 1985, 401). Nicholas Onuf (1989) introduced social constructivism to international relations theory to help overcome these shortcomings. It eventually gathered a large following among IR scholars with a series of influential articles and a book by Alexander Wendt (1987, 1992, 1994, 1995, 1999).

Social constructivism is an empirical approach to the study of international relations; it is empirical in that it focuses on the intersubjective ideas that define world politics. There are two camps of social constructivist scholars in IR theory. On the one hand, there are constructivists whose analysis is systemic in nature. They stress the importance of the international environment in shaping state interests and identities. The most prominent study in this camp is Wendt's Social Theory of International Politics (1999). His thesis is that the root of state interests and identities is the social interaction between states. Wendt also drives home the point that constructivism is not merely about "adding the role of ideas" to existing theories of IR. Rather, material power and state interest are fundamentally formed by ideas and social interaction. States in an anarchic system may possess military and other capabilities, which can be seen as potentially threatening by other states. Social interaction between states can also lead to more benign and friendly cultures of anarchy.

Another prominent work in this camp is Martha Finnemore's National Interests in International Society (1996). Like Wendt, her starting point is the definition of states' identities

\footnotetext{
${ }^{11}$ Social constructivism has its roots in philosophy and sociology. For further discussion see Giddens (1984).
} 
and interests. However, instead of looking at the social interaction between states, Finnemore focuses on the norms of international society and the way in which they affect state identities and interests. State behavior is defined by identity and interest, and identities and interests are defined by the norms of behavior embedded in international society. The norms of international society are ultimately transmitted to states through international organizations, which shape national policies by "teaching" states what their interests should be.

The second group of social constructivists put more emphasis on the domestic environment. This camp argues that

[a]lthough any understanding of world politics requires a theorization of the domestic and the systemic, there would be no systemic theory of world politics because world politics has no predominant system; it has subcultures, each of which can be understood only by examining how states constitute themselves in their societies. The answer to the question of who are enemies and friends begins at home. Finding out precisely how a state's identity affects the construction of its interests vis-à-vis another state demands that the social context in which that state's collection of identities is being discursively constructed be investigated as deeply and broadly as possible. This means exploring not only how that state's identities are produced in interactions with other states, but also how its identities are being produced in interaction with its own society and the many identities and discourses that constitute that society. (Hopf 2002, 294)

A major work on the domestic side of things is The Culture of National Security: Norms and Identity in World Politics (Katzenstein, ed. 1996). This book aims to drive home the point that domestic culture, norms, and identity matter in shaping interests and identities related to national security. In this context, many of the essays in the book put special emphasis on domestic norms. For example, Alastair Johnston takes up the case of Maoist China in order to see "how far ideational arguments can go in accounting for realpolitik behavior" (Johnston 1996, 217). He identifies a specific "hard realpolitik" strategic culture in the Chinese tradition that informs and shapes Chinese security policies. The argument he makes is that Chinese decisionmakers have "internalized this strategic culture" and that it "has persisted across vastly different 
interstate systems, regime types, levels of technology, and types of threat" (Ibid., 217).

In Culture Norms and National Security: Police and Military Power in Postwar Japan (1998), Katzenstein further develops a constructivist argument about the role of domestic norms in the area of national security. He emphasizes how the domestic normative structure can influence the identities, interests, and policies of states. The major puzzle that Katzenstein addresses is the Japanese shift from a militaristic foreign policy before 1945 to a pacifist foreign policy after the war. This analysis explains why there was a broad consensus favoring a militaristic foreign policy before the war and how the norms on which that consensus was based became contested as a result of the war.

Ted Hopf (2002) focuses on the domestic formation of identity in order to understand how national interests are defined and what foreign policies they lead to. By looking at Soviet and Russian foreign policy, Hopf seeks to provide "an account of how a state's own domestic identities constitute a social cognitive structure that makes threats and opportunities, enemies and allies, intelligible, thinkable, and possible" (16). Finally, another major work on the domestic side of things is Foreign Policy in a Constructed World (Kubalkova, ed. 2001). This book contains several essays that seek to explain the domestic roots of foreign policy interests and identities more generally. These essays collectively stress the importance that language plays in shaping the foreign policy interests and identities of states since speaking is the sharing of meaning.

Despite the debate about the relative importance of domestic versus international environments, constructivists are more united than divided. They all emphasize the importance of culture and identity, as expressed in social norms, roles, and understandings. They also agree that the social and political world is made up of shared beliefs, which must always be the starting 
point for analysis. Therefore, it is safe to say that social constructivists generally believe that meaningful behavior, or action, is only possible within a social context. It permits actors to develop their relations with, and understanding of, others through norms and practices. These norms comprise an identity by specifying the actions that will cause others to recognize that identity and respond to it appropriately. They provide meaning to action and to exercises of power.

It is from this general thesis where intelligence scholars that utilize the logic of social constructivism take-off. These individuals, especially Turner in his article "A Distinctive U.S. Intelligence Identity" (2004), argue that U.S. intelligence is a highly malleable product of historical processes. These are the result of the prevailing discourse in society that reflects and shapes beliefs and interests leading to accepted norms of behavior. Intelligence norms are the product of the dominant strategic culture, its subset, U.S. strategic values, and the dominant political culture (Ibid., 43). These provide meaning to actions and to exercises of power. Intelligence agencies ultimately internalize these norms and practices causing the IC to have a particular kind of identity. They specify the kinds of actions it takes for others to recognize it as intelligence. In the end, intelligence norms form the basis for identity that is a reasonably accurate guide to institutional behavior (Ibid., 49).

Although intelligence experts have assembled a rather long list of the key traits that make up the U.S. intelligence identity (Sims 2005, 36-37), fourteen norms are predominant. ${ }^{12}$ First, there are norms that provide U.S. intelligence with the tools to do its job. These instrumental norms are secrecy, intelligence exceptionalism, flexible accountability, policy support, and the

\footnotetext{
${ }^{12}$ The fourteen U.S. intelligence norms, according to Turner (2004), are as follows: institutional survival; secrecy; intelligence exceptionalism; ambiguous mandate; confederal structure; flexible accountability; separation of intelligence from policy; policy support; "can do" attitude; primacy of intelligence analysis; emphasis on current intelligence; and the provision of accurate, timely, and relevant intelligence.
} 
"can do" principle. Next, regulative norms, such as a fragmented structure, separation of intelligence from law enforcement, and separation of intelligence from policy provide a measure of control to prevent excesses. Finally, norms like the importance of intelligence analysis, and the provision of accurate, timely, and relevant intelligence straddle the regulative and instrumental divide. They exist as guides for doing the intelligence task well, while also serving as standards for the performance of that task.

Intelligence norms are particularly useful for trying to explain patterns of behavior among U.S. intelligence agencies. For one, these norms are guideposts for performance. Striving toward these guideposts is part of the intelligence identity. This makes the intelligence norms the "ideal type" actions that the non-intelligence world readily recognizes as being attributes to the realm of U.S. intelligence (Turner 2004, 57). As Sims (2005) points out, the U.S. intelligence identity "informs the way U.S. decision makers, analysts and intelligence producers think about intelligence" (33). Intelligence norms are also tools for attaining political goals in the most efficient way in a given political context (Turner 2004, 57). They are the templates for an intelligence identity that exists within a political culture that is unsure about what role the IC should play in American society.

In addition to helping explain patterns of behavior among U.S. intelligence agencies, the dominant intelligence norm, institutional survival, vindicates the realist argument. It strongly suggests that most IC behavior will be conducted in accordance with the propositions of the bureaucratic politics model. As Turner (2004) posits,

American agencies that engage in intelligence have one attribute in common with all other governmental organizations: they are bureaucracies seeking to maximize what Max Weber called "professional information" intended to increase their stature relative to others; to promote their bureaucratic interests; and to survive in the political marketplace that places so much value on these attributes. Probably the dominant norm of U.S. intelligence, it is not confined to the intelligence establishment alone. (49) 
The social constructivist approach to understanding the behavior of U.S. intelligence agencies is quite compelling. It accounts for their competitive nature, which leads to dissimilar behaviors between and among them. It also helps explain why they also act similarly in several ways. Thus, the U.S. intelligence identity, and the norms that comprise it, seemingly account for the IC agencies interests and preferences. It provides the basis for important intelligence work (Ibid. 58). ${ }^{13}$ It is for these reasons that the social constructivist approach appears to be an appropriate substitute for the bureaucratic politics model and its ontological reductionism.

\section{The Need to Enrich the U.S. Intelligence Identity with Insights about Cognition}

According to Wendt (1999), a constructivist approach to politics is "at base cognitive rather than behavioral" (394). Cognitions are the beliefs and reasoning processes that individuals, groups, societies, and other entities use to understand and make sense of the world. They matter because they underlie all political behavior and form the foundation for how power and interests are understood (Young and Schafer 1998, 84). ${ }^{14}$ This means that constructivism has to be enriched with insights about cognition (Wendt 1999, 394). However, very few constructivist scholars have followed this recommendation so far. ${ }^{15}$ This is because "the emphasis on the social construction of identities and worldviews tends to give priority to the social and cultural sources of identity formation [thereby downplaying] the psychological needs that are satisfied by those identities and that systematically shape the social construction of identities" (Levy 2003, 273). Furthermore, “social psychological needs...constrain the

\footnotetext{
${ }^{13}$ It also offers insight into why intelligence failures occur. According to Turner (2004), "the U.S. intelligence identity reflects the ambiguity that Americans have about secret intelligence. United States citizens want the intelligence function to serve the national interest, but in ways that are palatable to the country's democratic sensibilities. Ultimately, this kind of identity, though providing the basis for important intelligence work, becomes also a prescription for intelligence failure" (58).

${ }^{14}$ See Levy (2003), Simon (1957), Tetlock (1998), and Vertzberger (1990) for further discussion on how cognitions underlie political behavior

${ }^{15}$ Notable exceptions include Malici (2006) and Walker (2004).
} 
construction of identities in a way which the analysis of cultural or institutional variables do not capture" (Goldgeier 1997, 142).

Constructivists writing about intelligence must incorporate insights about the beliefs and reasoning processes of the IC into their arguments. Otherwise, their explanations are incomplete and are unable to fully account for patterns of behavior among the intelligence agencies' given that their actions are not exogenously defined. Constructivists writing about intelligence do make note of the beliefs that result from prevailing discourse in society (Turner 2004, 43). This is because "although not all Americans think alike, it is arguable that they do share certain values and norms of behavior that transcend partisan politics" (Sims 2005, 34). Nevertheless, a glaring set of cognitions that is missing from the constructivists' argument is the set of beliefs that the IC holds about world politics-its worldview. ${ }^{16}$ This prevents these scholars from being able to fully account for shared behaviors related to foreign intelligence activities, such as the intense effort of all IC agencies to protect their sources and methods from foreign actors.

The term "worldview" is most often defined as the overall perspective from which one sees and interprets the world (Koltko-Rivera 2004). It comes from the German word Weltanschauung and is a concept fundamental to German philosophy and epistemology. ${ }^{17}$ It is also one of the most important concepts in cognitive psychology, which is a school of thought in psychology that examines internal mental processes such as language, memory, and problem solving. This is due to fact that it has a powerful effect on cognition and behavior. As Mark E. Koltko-Rivera (2004) explains,

A worldview is a way of describing the universe and life within it, both in terms of what is and what ought to be. A given worldview is a set of beliefs that includes limiting statements and assumptions regarding what exists and what does not (either in actuality, or in principle), what objects or experiences are good or bad, and what objectives,

\footnotetext{
${ }^{16}$ World politics refers to foreign affairs and global issues among state and non-state actors.

${ }^{17}$ Welt is the German word for "world" and Anschauung is the German word for "view" or "outlook."
} 
behaviors, and relationships are desirable or undesirable. A worldview defines what can be known or done in the world, and how it can be known or done. In addition to defining what goals can be sought in life, a worldview defines what goals should be pursued. Worldviews include assumptions that may be unproven, and even unprovable, but these assumptions are superordinate, in that they provide the epistemic and ontological foundations for other beliefs within a belief system. (2)

There are strong reasons why the IC's worldview is likely to be an essential component of its identity. First, practically every type of organization has a worldview (Borhek and Curtis 1975; Hatch 2004; Schein 1996, 2004). In fact, a number of studies have acknowledged the existence of the IC's worldview (e.g., Halperin 1974; Heuer 1999; Immerman 2008; Jackson and Siegel 2005; Johnston 2005; Wirtz 1991). An organization's worldview is the set of fundamental assumptions or beliefs that provide a core set of values, which form the basis for its goals and priorities. Therefore, organizational values are beliefs and ideas about what kinds of goals members of an organization should pursue and ideas about the appropriate kinds or standards of behavior organizational members should use to achieve these goals. These values ultimately help define a system of norms and expectations specific to the organization-its culture. The levels of organizational culture, according to Edgar H. Schein (2004), ${ }^{18}$ are shown in Figure 1.1.

Second, the IC's worldview is in all likelihood a part of its raison d'etre. ${ }^{19}$ As I have already pointed out, the foremost concerns of the IC are the "actions, policies, and capabilities of other nations and of important non-state groups" (Lowenthal 2006, 5). The IC's heavy focus on foreign entities is especially important in today's world given that U.S. national security policymakers have a multitude of concerns. They are concerned with the capabilities and plans of al-Qaeda and other terrorist groups; political and military developments in Afghanistan, Iraq, and Pakistan; the status of Iran and North Korea's nuclear and/or missile programs; the foreign

\footnotetext{
${ }^{18}$ Schein is one the foremost experts on organizational culture.

${ }^{19}$ Raison d'etre is a French phrase meaning "reason for being."
} 
and military policies of China and Russia; and failed states, especially in Africa. This is just a small sample of the national security issues that the intelligence agencies' are currently grappling with.

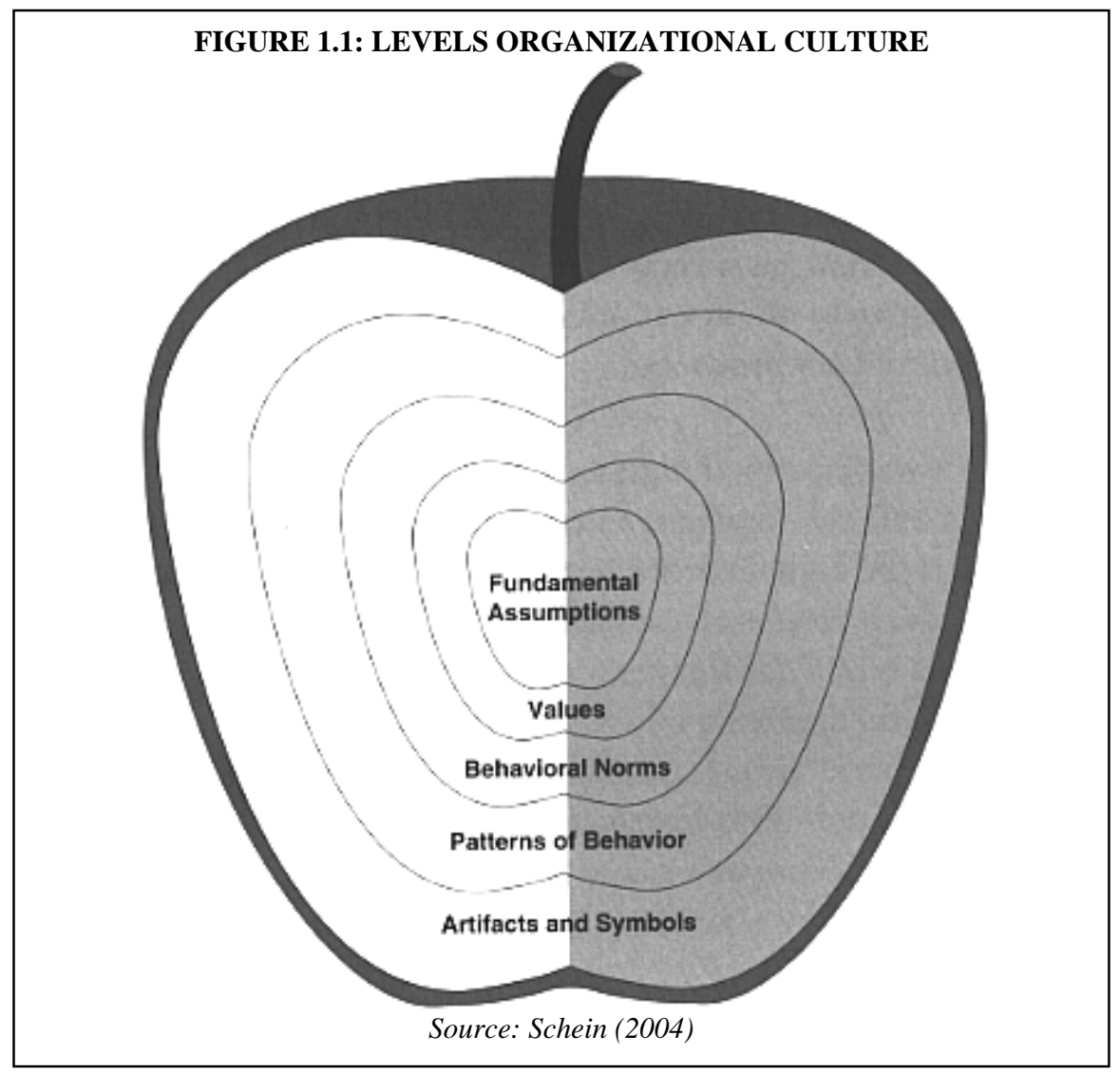

Finally, intelligence has historically played a key role in shaping the policies of the U.S. toward other countries. For instance, intelligence changed Ronald Reagan's policy toward the "Evil Empire," and strongly influenced President George H.W. Bush's decisions regarding Desert Storm (Andrew 1995). Moreover, intelligence has been very useful in a variety of conflict situations; it has provided advanced warning of impending hostile action against the U.S.; it has helped assess whether other nations are in compliance with various international obligations, and it has aided decisions on the suspension or resumption and of foreign aid, the employment of trade sanctions and embargos, and attempts to block the transfer of commodities 
related to nuclear or ballistic missile proliferation (Richelson 2007, 8-12).

This discussion suggests that the cognitions the IC holds about world politics are partly responsible for shaping its identity. It also suggests that its identity is meant to help satisfy these beliefs. This indicates that U.S. intelligence norms are likely to derive, in part, from the IC's belief system about world politics. Therefore, by incorporating the IC's worldview into the U.S intelligence identity, the constructivist explanation will be strengthened. It will also help provide better understanding of patterns of behavior among the intelligence agencies'. This is crucial since it may facilitate cooperation among them, which could help prevent intelligence failure.

\section{Defining the Worldview of U.S. Intelligence}

A particular set of cognitions, such as a worldview, represents a belief system. A belief system "is a configuration of ideas in which elements are bound together by some form of functional interdependence" (Converse 1964, 207-208). ${ }^{20}$ Interdependence is the probability that a change in the status of one belief will psychologically require some compensating change(s) in the status of other beliefs (Hopf 1994, 18). Belief systems are hierarchically organized with central, intermediate, and peripheral beliefs (Beck 1995; Converse 1964; Dawson 1979; Hamilton and Mineo 1996, 1999). Central beliefs refer to the basic assumptions and values from which everything else in the belief system is ultimately derived (Beck 1995; George 1969; Holsti 1977). Thus, core beliefs are the most fundamental level of beliefs. They establish broad guidelines for behavior and are generally considered to be absolutistic and rigid (Jervis 1976; Nisbett and Ross 1980). Intermediate beliefs are related rules, attitudes, and assumptions that follow from the core beliefs. These beliefs function to provide normative direction as well as additional analytical concepts. Intermediate beliefs ultimately generate "automatic thoughts," which are also known as peripheral beliefs. Automatic thoughts are evaluative cognitions that

\footnotetext{
${ }^{20}$ This is the definitive definition within the literature on belief systems.
} 
occur in response to a particular situation. They are spontaneous and not the result of deliberate extended thinking or logical reasoning. Nevertheless, they are still indirectly connected to central beliefs.

Since core beliefs hold all the other beliefs in the system together, this dissertation defines the worldview of U.S. intelligence as the set of core cognitions that the IC holds about foreign affairs and global issues among state and non-state actors (world politics). Core cognitions in this context refer to the most fundamental beliefs that the IC generally holds about world politics. ${ }^{21}$ They are the underlying assumptions that the intelligence agencies' most often make when confronting issues related to world politics. Although the various intelligence agencies within the IC are likely to have distinct worldviews as well (Schein 2004), these subworldviews ultimately adhere to the core belief system that U.S. intelligence holds about world politics (Builder 1989; Howard-Grenville 2006; Morgan 1997; Schein 2004). ${ }^{22}$ That is, the core beliefs that the intelligence agencies' hold about world politics mirror (reflect) the core cognitions that comprise the IC's worldview. This is because, as social constructivists posit, the IC agencies have internalized U.S. intelligence norms, which define collective expectations for the proper behavior of the intelligence agencies' in fulfilling their missions. Finally, the worldview of U.S. intelligence is considered to be overarching given that research on cognition suggests that the IC's worldview is partly responsible for shaping its identity and its identity is meant to help satisfy the beliefs that comprise it.

\footnotetext{
${ }^{21}$ I discuss the continuity of the IC's worldview in chapter three of this dissertation.

${ }^{22}$ For example, according to Builder (1989), the worldview that is attributed to the U.S. Department of Defense (DOD) comprises the distinct worldviews of the different military services and the corps of civil servants assigned to each service agency. Yet, all of these sub-worldviews ultimately adhere to the core belief system of the DOD.
} 


\section{Research Question}

Unfortunately, no study has even considered what the worldview of U.S. intelligence might look like let alone where it originates from. This means that the cognitions that comprise the IC's worldview have not yet been identified. Existing studies, such as those mentioned above, only acknowledge its existence. Moreover, this is often done hastefully. This situation exists largely because political scientists have paid scant attention to intelligence issues directly. The academic literature on intelligence mostly consists of books and journal articles written by former intelligence professionals, journalists, and historians. Consequently, beyond the research on the politics of U.S. intelligence, most studies on intelligence are not organized around some form of theoretical framework.

Given the preceding, researchers are currently unable to incorporate the IC's worldview into its identity. Granted, a researcher could simply assume what cognitions comprise the set of core beliefs that U.S. intelligence holds about world politics and then introduce them into the IC's identity. A researcher could extrapolate them from his or her knowledge base and/or professional experience, but this would harm the objectivity of analysis since it would be driven, in part, by opinions and prejudices. Therefore, prior to incorporation, the set of cognitions that comprise the IC's worldview have to be first tested against observations of reality to "liberate inquiry from bias, prejudice, and just plain muddleheadedness" (Hoover and Donovan 2001, 1). The IC's worldview can then be inserted into the U.S. intelligence identity to generate objective insights.

This dissertation utilizes the social scientific method to provide an answer to the following research question: What is the worldview of U.S. intelligence ${ }^{23}$ It is my hope that by

\footnotetext{
${ }^{23}$ The social scientific method is used to investigate social phenomena, acquire new knowledge, or correct and integrate previous knowledge (Hoover and Donovan 2001). It is based on gathering observable, empirical, and
} 
providing an answer to this research question, I will be able to facilitate several contributions to the literature on intelligence. One potential contribution is an increased understanding of shared behaviors among the various intelligence agencies. Furthermore, the psychological perspective that I employ could highlight the cognitive biases that have led to many intelligence failures (Heuer 1999). It might also highlight the reforms that are most useful for preventing intelligence failures. For instance, competitive analysis can reduce the power of preconceptions (Austin 1985, 216). All of these potential contributions, among others, will be discussed further in the final chapter of this dissertation.

\section{Chapter Summary and Dissertation Plan}

This chapter reviewed and critiqued the literature on the politics of U.S. intelligence. The purpose of this was to clarify the importance of, and the reasoning behind, taking a psychological approach to understand why IC agencies do act similarly sometimes. In the first section, I defined the concept of intelligence for the purposes of this study. The second section presented the scholarly literature on the politics of U.S. intelligence, which aims to understand where the interests and preferences of the various agencies that make up the IC originated. This research program utilizes organization theory and social constructivist theory. The former is much more ubiquitous in the literature than the latter. However, because the bureaucratic politics model suffers from ontological reductionism, it cannot explain all of the intelligence agencies' behaviors. The social constructivist approach, which has been utilized by intelligence scholars to create a list of orientations and preferences in the IC that would define its "identity," does a better job of explaining where the interests and preferences of IC agencies come from, especially when it comes to similar patterns of behavior.

measurable evidence subject to specific principles of reasoning. The social scientific method consists of the collection of data through observation and experimentation, and the formulation and testing of hypotheses. 
In the next section, it was explained that social constructivists who write about intelligence do not go far enough in their reasoning since they ignore the set of beliefs that the IC holds about world politics - its worldview. I went on to argue that the worldview of U.S. intelligence must be incorporated into the IC's identity since it is likely to form the foundation for patterns of behavior related to foreign intelligence activities among the several agencies that make up the IC. After defining the IC's worldview, I presented this dissertation's primary research question, which asks what the worldview of U.S. intelligence is. I argued that answering this question is a necessary first step towards incorporating the IC's worldview into its identity.

The remainder of this dissertation will be organized as follows. Chapter two explains how this dissertation conceptualizes the worldview of U.S. intelligence. After sifting through research on political psychology and foreign policy analysis, I argue that the IC's worldview is best conceptualized as the five philosophical beliefs in an operational code. The operational code is a set of philosophical beliefs about the nature of political life and the predictability and controllability of political outcomes, and instrumental beliefs about the best to pursue goals and calculate risks. The selection of the operational code construct, and the philosophical beliefs within it, is due to several reasons. For one, the operational code is a valid and longstanding research tool for studying belief systems. Furthermore, because of aggregation effects, it can be used as a group measure. Finally, in terms of theory, constructivism and the operational code construct go well together.

Chapter three presents my theory and hypotheses. The theory of this dissertation is that the IC's worldview underlies several U.S. intelligence norms. As this chapter explained, U.S. intelligence norms derive in part from the core beliefs that U.S. intelligence agencies collectively 
hold about world politics. After presenting my theory, I posit six hypotheses. The first states that the worldview of U.S. intelligence is specific and longstanding. The other five propositions focus on the philosophical beliefs in an operational code.

Chapter four explains how I will test my hypotheses. The automated content analysis system Verbs in Context System (VICS) will be used to score attribution patterns in excerpts from declassified National Intelligence Assessments (NIEs). Declassified NIEs are the only publically available source that can reflect and aggregate the beliefs of all IC agencies with reasonable accuracy. After VICS codes each NIE, I will run several statistical tests. This allows me to assess the statistical significance of my hypotheses. After presenting my findings in chapter five, the dissertation concludes (in chapter six) with a discussion on its theoretical and practical implications. 


\section{CHAPTER TWO}

\section{CONCEPTUALIZING THE WORLDVIEW OF U.S. INTELLIGENCE}

This chapter explains how this dissertation conceptualizes the worldview of U.S. intelligence, which refers to the set of core cognitions that the IC holds about foreign affairs and global issues among state and non-state actors (world politics). Concepts are the mental categories that help us classify objects, events, or ideas. However, many concepts relate to reality only vaguely. For example, "in common usage, the term "star" covers a multitude of objects, big and small, hot and cold, solid and gaseous" (Hoover and Donovan 2001, 14). The core cognitions that the IC holds about world politics could also refer to a great number of beliefs or reasoning processes. As Richard K. Herrmann (2003) notes, "to describe an actor's view of the world or perception of another actor is a complicated task. [An actor] may have many beliefs about another actor, including, among other things, what the actor values, what role it plays in a particular region, and how its economy works or political system functions" (286).

Notwithstanding, each object, event, or idea has a set of common relevant features. Conceptualization or concept formation refers to the process of specifying and refining concepts for the purpose of empirical research (Brady and Collier 2004, 279). ${ }^{1}$ It is the process of identifying a concept's characteristics so that evidence which proves or disproves the concept's existence can be observed. In this chapter, I specify the exact cognitions that characterize the IC's worldview. I achieve this by comparing and contrasting the most salient theoretical frameworks that researchers of world politics use to conceptualize worldviews. This allows me to easily identify the cognitions that are most fundamental to actors' belief systems about world politics.

\footnotetext{
${ }^{1}$ Brady and Collier note that political theorists do engage in concept formation for other purposes. However, in general, it refers to the empirical application of concepts.
} 
This chapter is organized as follows. In the first section, I explain that the IR subfield of foreign policy analysis (FPA) contains four theoretical frameworks that I could potentially use to conceptualize the worldview of U.S. intelligence. I then briefly review these four theoretical frameworks and explain how they are used by researchers to conceptualize an actor's worldview. In the section that follows, I argue that even though each approach has its benefits, the best way to conceptualize the worldview of U.S. intelligence is in the form of an operational code, specifically as the five philosophical beliefs within it. This is due to several reasons, but mostly because the operational code construct is one of the most widely used and general set of cognitions. Operational code analysis also surpasses the other theoretical frameworks that I discuss when it comes to its ability to aggregate the core beliefs that the various IC agencies hold about world politics. The chapter concludes with a summary of the preceding sections.

\section{Foreign Policy Analysis and Political Psychology}

There are a variety of academic disciplines that study worldviews. This list, to name a few, includes philosophy, psychology, sociology, and history. Nevertheless, only the political science subfield of IR contains a substantial amount of research on actors' belief systems about world politics. Specifically, it is it the IR subfield of foreign policy analysis that contains scores of studies on the core cognitions that make up a political actor's worldview. FPA is the systematic study of and research into the processes and theories of foreign policy or, in other words, it is the study of how states make foreign policy. The explanatory premises of FPA are those factors that influence foreign policy decision making and foreign policy decision makers (Hudson 2005). Thus, two hallmarks of FPA scholarship are that it views any explanation of foreign policy as being multifactoral and multilevel. Explanatory variables from all levels of analysis affect the decision making process. Foreign policy analysts also utilize insights from a 
variety of intellectual disciplines, such as anthropology, economics, organizational behavior, and sociology.

The discipline which FPA borrows most heavily from is psychology, particularly political psychology. ${ }^{2}$ Psychological characteristics have been an integral part of FPA research since its inception as a field of inquiry in the mid-1950s (Schafer 2000, 511). Indeed, many of the classics in this literature give psychology a prominent place in their analyses (Jervis 1976; Leites 1951; Smith 1968; Sprout and Sprout 1965). Psychological variables are

central to the explanation of individual beliefs, preferences and decisions, and to decision-making in small groups and organizations as well as states. By shaping foreign policy, psychological variables affect outcomes at the dyadic and systemic levels. They also affect public opinion, nationalism, identity formation, and other variables originating at the societal level. (Levy 2003, 253)

FPA scholars have developed many cognitive constructs to help explain foreign policy decision making and international relations (e.g., Holsti 1967; Jervis 1976; Cottam 1977; George 1980; Bueno de Mesquita and Lalman 1986; Kim and Bueno de Mesquita 1995). This is because "whether models of decision making emphasize rational information processing and optimizing choices or concentrate on cognitive biases and various types of reasoning, their foundation is rooted in claims about a subject's perceptions, that is, the foreign policy decision maker's construction of reality" (Herrmann, et. al. 1997, 404). In turn, identifying belief systems, including worldviews, is often the first task researchers undertake to best capture strategic calculations. As a result, there are several analytical approaches in FPA that could provide the necessary theoretical framework to conceptualize the IC's worldview. There are four approaches that are especially relevant. These are cognitive mapping, image theory, leadership

\footnotetext{
${ }^{2}$ At the most general level, political psychology is an application of what is known about human psychology to the study of politics (Sears, Huddy, and Jervis 2003). It draws largely on theory and research on personality, psyopathology, social psychology, developmental psychology, cognitive psychology, and intergroup relations.
} 
trait analysis (LTA), and operational code analysis (OCA).

\section{Cognitive Mapping}

In behavioral psychology, a cognitive map is a mental model, or belief system, that people use to perceive, contextualize, simplify, and make sense of otherwise complex problems (Downs and Stea 1973; Kitchin 1994; Tolman 1948). It is a cognition composed of a series of psychological transformations by which an individual acquires, codes, stores, recalls, and decodes information about the relative locations and attributes of phenomena in their everyday or metaphorical spatial environment. Cognitive maps are a method people use to structure and store spatial knowledge. ${ }^{3}$ This allows the "mind's eye" to visualize images in order to reduce cognitive load and enhance the recall and learning of information. It can also be used as a metaphor for non-spatial tasks, which is where people performing tasks involving memory and imaging use spatial knowledge to aid in the processing of the task.

Cognitive mapping was introduced to FPA in the early 1970s to improve the quality of the decision making processes of U.S. foreign policymakers (Axelrod 1972, 1976a, 1976b; Shapiro and Bonham 1973). In developing the cognitive map, Robert Axelrod built on five earlier bodies of work: psycho-logic (Abelson and Rosenberg 1958), causal inference (Simon 1957; Blalock 1964), graph theory (Maruyama 1963; Cartwright and Harary 1965; Harary, Norman, and Cartwright 1965), evaluative assertion analysis (Osgood, Saporta, and Nunnally 1956), and decision theory (Luce and Raiffa 1957). By combining these five bodies of scholarship, Axelrod's essential idea was to organize all of a decision maker's causal beliefs in an accessible, visual manner-a network of concepts connected by their causes. Therefore, a cognitive map would simply be a representation of a worldview consisting of nodes (representing values, beliefs, attitudes, and actions) and links (representing perceived causal and

\footnotetext{
${ }^{3}$ Spatial knowledge refers to the symbols by which knowledge is expressed.
} 
other relationships between the nodes). Several other scholars quickly took to Axelorod's idea, which led to several additional pioneering studies. These include Bonham and Shapiro (1976), Roberts (1976), and Hart (1976).

Since this time, the cognitive map has become a very useful tool for modeling and studying worldviews and decision processes. It has benefited from the initial creation of fairly well-defined and documented procedures (Young and Schafer 1998, 76). Indeed, "the basic procedure for constructing cognitive maps has not changed significantly since 1976" (Ibid., 76). Furthermore, the Cognitive Process Model (CPM), ${ }^{4}$ which Matthew Bonham and Michael J. Shapiro $(1973,1976)$ developed to move the use of cognitive maps beyond purely qualitative analysis, helps researchers identify the strongest connections in the cognitive map. This is important since the strongest connections are the most important guides to reasoning (Young 1996). The cognitive map also helps researchers illuminate the fact that worldviews are generally unique to each individual. Individuals "map" their environments in their heads, and such mapping suggests ways for individuals to achieve their preferences and realize their goals (Ibid.).

\section{Image Theory}

The effects of images on foreign policy decision making have been discussed in FPA literature for many years (Young and Schafer 1998, 79). A theory of national images was first posited by Kenneth Boulding in $1959 .^{5}$ He defined an image as "the total cognitive, affective,

\footnotetext{
${ }^{4}$ The CPM model has five steps. These are the amplification of applicable concepts (matching concepts in the situation to the belief system and generating lists of "highlighted concepts"), the search for antecedents (finding all prior causes of the highlighted concepts and adding them to the list of stored paths), the search for consequences (creating a set of consistent paths by going through the map from highlighted concepts to terminal concepts; storing the paths; and finding the most central path(s), the search for policy alternatives (finding all policy concepts that are directly related to this central path), and the choosing of a policy alternative (selecting the policy concept that leads to the maximum gain in an ordered set of values).

${ }^{5}$ Boulding first talked about the effects that images of the self and of others could have on the foreign policymaking process in his 1956 book The Image.
} 
and evaluative structure of the behavior unit, or its internal view of itself and the universe," and argued that "the images which are important in international systems are those which a nation has of itself and of those other bodies in the system which constitute the international environment" (Boulding 1959, 120-121). Images of the self and of others shape strategic decisions. In particular, Boulding felt that the perceived hostility or friendliness and perceived strength or weakness of a unit were the central features of a subject's image of that unit (Ibid., 124-125). Subsequent studies disassembled Boulding's general notion into different types of images (e.g., Holsti 1967).

It was not until the mid-1970s, however, that the effects of images on foreign policy decision making became a cohesive research program. This began with the work of Richard Cottam (1977) and was expanded extensively by Martha Cottam (1986, 1992), and Richard Hermann (1984). ${ }^{6}$ These scholars argued that decision makers need to simplify reality in order to make its complexity manageable. The specific simplifications that are chosen often serve the motivational needs of individuals to justify actions or to balance other beliefs. Once formed, these cognitive constructions become filters through which information passes and upon which policy choices are advocated. Consequently, images can have a causal effect on behavior.

In addition to positing a general thesis on the effects of images on world politics, these scholars also identified the most pervasive images that decision makers use to understand international affairs (M. Cottam 1994; R. Cottam 1977; Herrmann 1985; Holsti 1967). These are the enemy, ally, imperial, and colonial images. Given that images are used to serve motivational needs, these four types of images obviously play a vital role in shaping belief systems about world politics. Moreover, these images can constitute a political actor's worldview given that

\footnotetext{
${ }^{6}$ Unlike previous studies on image theory, these works were grounded in ideas and concepts emerging from cognitive psychology.
} 
the perception of another actor defines the view of that actor (Herrmann 2003, 288). These prototypical images "provide categories that allow people to sort and make sense of the political environment and their relationships in it" (Ibid., 294). In other words, these four images help individuals make sense of the world in a parsimonious fashion. This is undoubtedly the essence of a worldview.

\section{Leadership Trait Analysis}

Leadership trait analysis provides information about different aspects of a leader's personality. This information can then be used to assess a leader's leadership style, which refers to the ways in which leaders relate to those around them and how they structure interactions and the norms, rules, and principles they use to guide such interactions (Hermann 2005, 181). Leadership styles are important given that "the particular leadership style that political leaders adopt can affect the manner in which they deal [with dilemmas] and, in turn, the nature of the decision-making process" (Ibid., 181). Several FPA studies have examined the empirical relationship between leadership styles and political behavior. These include Bass (1981); Driver (1977); Druckman (1968); Byars (1973); Hermann (1980, 1984, 1985); Hermann and Hermann (1989); Hermann and Kogan (1977); Hermann and Preston (1994): Karbo and Hermann (1998); McClellan (1975); Lefcourt (1976); Walker (1983); and Winter (1992).

Margaret Hermann (2005) argues that leadership style is generally a function of responsiveness to constraints, openness to information, and motivation (181-184). These three variables suggest whether a leader is going to be generally sensitive or insensitive to the political context and the degree to which he or she will want to control what happens or be an agent for the viewpoints of others. Hermann also argues that the aforementioned body of scholarship suggests that seven personality traits are particularly useful in assessing leadership style. These 
are the belief that one can control or influence what happens, the need for power and influence, conceptual complexity, ${ }^{7}$ self-confidence, the tendency to focus on problem solving and accomplishing something versus maintenance of the group and dealing with others' ideas and sensitivities, general distrust or suspiciousness of others, and the intensity with which a person holds an in-group bias. Hermann concludes that by doing a trait analysis of these seven dimensions of personality, it becomes possible for a researcher or analyst to develop a profile of the leadership style a particular leader is most likely to exhibit (211).

This framework, which is known as the LTA approach, is useful for conceptualizing worldviews for a couple of reasons. For one, it is made of up cognitions that can provide much insight into how a political actor views their external environment. For example, assessing ingroup bias and the general distrust of others can assist us in learning if the political actor thinks the world is full of enemies and in constant conflict. Furthermore, conceptual complexity allows the researcher to measure the degree to which individuals are able to see ambiguity in their external environment. Another reason why the LTA approach is useful for determining a political actor's worldview is that the seven personality traits found within it reflect the language of "first impressions" (Winter 2003, 115). They reflect the adjectives and adverbs of everyday language that are used to describe other people. When taken together, these reasons suggest that LTA offers several cognitions that can be used to conceptualize a worldview.

\section{Operational Code Analysis}

Operational code analysis focuses on the beliefs of political leaders as causal mechanisms in explaining foreign policy decisions (Walker, Schafer, and Young 1999). The operational code has a long, rich history in FPA (Young and Schafer 1998, 74). Indeed, "when we look at the

\footnotetext{
${ }^{7}$ Conceptual complexity refers to the ability to differentiate things and people in one's environment (Suedfeld and Rank 1976; Young and Schafer 1998; and Wallace and Suedfeld 1988)
} 
work on operational codes, we are looking at the most widely used concept relating to the link between belief systems and international relations" (Smith 1968, 11). Nathan Leites (1951, 1953) originally developed the operational code to help U.S. policymakers make sense of Soviet bargaining behavior. In his work on the Soviet Politburo, Leites argued that ideology, socialization, and leadership processes result in a consistent and identifiable set of behavioral patterns in Soviet foreign policy. Leites also included the psychoanalytic and psychocultural sources of Soviet actions in his analysis. He believed that cognition, character, and culture comprise an operational code.

Although Leites work was groundbreaking, the complexity of his approach proved frustrating to many. This led Alexander George $(1969,1979)$ and others (Holsti 1977 and Walker 1983,1990$)$ to argue that the operational code construct should simply be a political belief system in which some elements (philosophical beliefs) guide the political actor's diagnosis for action in the political universe and others (instrumental beliefs) prescribe the most effective strategy and tactics in achieving political goals. The political actor's belief system in this approach thus becomes a mechanism that reflects unspecified personality biases and societal influences. This forms a heuristic that can be applied to any political actor by answering the ten questions that follow regarding specific philosophical and instrumental beliefs. The answers to these ten questions specify a model of instrumental rationality bounded by a belief system (George 1969, 1979).

The following are the five philosophical questions:

1. What is the "essential" nature of political life? Is the political universe essentially one of harmony or conflict? What is the fundamental character of one's political opponents?

2. What are the prospects for the eventual realization of one's fundamental values and aspirations? Can one be optimistic, or must one be pessimistic on this score; and in what respects the one and/or the other? 
3. Is the political future predictable? In what sense and to what extent?

4. How much "control" or "mastery" can one have over historical development?

What is one's role in "moving" and "shaping" history in the desired direction?

5. What is the role of chance in human affairs and in historical development?

The five instrumental questions are:

1. What is the best approach for selecting goals or objectives for political action?

2. How are the goals of action pursued most effectively?

3. How are risks of political action calculated, controlled, and accepted?

4. What is the best "timing" of action to advance one's interests?

5. What is the utility and role of different means for advancing one's interests?

Of the ten beliefs that comprise an operational code, the five philosophical beliefs are most important for determining worldviews. They focus on the political universe in which the political actor finds oneself and the nature of the "other" in this environment. This is in contrast to the five instrumental beliefs, which represent the image of the "self" and the best strategies and tactics one could employ to achieve one's ends. Therefore, the philosophical beliefs in an operational code, rather than the instrumental ones, comprise a political actor's worldview. Focusing on the philosophical beliefs in an operational code is especially advantageous given that hypothesizing the instrumental beliefs. Strategies vary in direction while tactics vary in intensity (Schafer and Walker 2006a, 35).

\section{What is the Best Way to Conceptualize the Worldview of U.S. Intelligence?}

Each of these research programs on cognition has its advantages for conceptualizing worldviews. Cognitive mapping allows the researcher to illuminate the idiosyncratic nature of belief systems and worldviews; image theory provides a simple classification scheme for describing a political actor's worldview; LTA provides a wide range of indicators that can explain how an actor perceives their external environment; and the operational code construct is one of the most widely used and general set of cognitions about world politics (Winter 2003, 123). Each research program has its disadvantages as well. Cognitive mapping does not present 
a theoretical framework that can be easily replicated; image theory's simple classification scheme could lead to omitted variable bias; ${ }^{8}$ most LTA indicators are not appropriate for conceptualizing a political actor's worldview, and OCA researchers have yet to identify which of the ten beliefs in an operational code are most important. These advantages and disadvantages suggest that Richard K. Herrmann's claim about the complicated nature of describing worldviews is indeed warranted.

To overcome this complication, two questions need to be answered when determining which cognitive construct will best conceptualize the IC's worldview. First, in terms of theory, which construct matches best with social constructivism? According to Thies (2006), "constructivism and the operational code construct go well together theoretically speaking" (232). Furthermore, Malici (2006) and Walker (2004) have demonstrated that constructivist theory can be investigated fruitfully with the operational code. This is because "the operational code construct expects beliefs to have mirrroring, steeering, learning effects, thus serving as causal mechanisms that link macro and micro-structural levels in the construction of identity" (Thies 2006, 232). Granted, cognitive mapping, image theory, and leadership trait analysis probably have their places within constructivist theory. However, no research has been done to substantiate this.

The second question asks which cognitive construct can be used as a group measure. All four research programs discussed in the previous section, as well as psychological analyses in general, focus on the individual level of analysis. Indeed, a central assumption of these four research programs is that individual leaders matter in shaping the foreign policy of states and that the beliefs they hold act as causal mechanisms in understanding why they chose a certain foreign

\footnotetext{
${ }^{8}$ Omitted variable bias refers to the bias that occurs when an independent (explanatory) variable is not included in a model when it should have been.
} 
policy decision. This is problematic for conceptualizing the IC's worldview since U.S. intelligence is made up of a collection of agencies whose overall structure is best characterized as a confederation due to its lack of a well-defined, unified leadership and governance structure (Turner 2004, 51). Therefore, the crux of the problem is selecting the theoretical framework that can best aggregate the fundamental beliefs that the various IC agencies hold about world politics.

Fortunately, FPA researchers and analysts do recognize the fact that individuals rarely make decisions without at least some input from others in the process (Schafer 2000, 513). "Among those making [foreign] policy are prime ministers, presidents, party secretaries, standing committees, military juntas, cabinets, bureaucracies, legislatures, loosely structured revolutionary coalitions" (Hermann 2001, 47). However, only operational code analysts have developed a procedure that takes this observation into account when discerning a "state's" operational code. For example, Leites' (1951) seminal work on the operational code sought to explain the foreign policy of the Soviet Politburo. The "rules" he discerned purported to explain the behavior of the state as a coherent entity. More recent studies, such as Feng (2007), Marfleet (2000), Schafer and Crichlow (2000), Schafer and Walker (2006a), and Walker and Schafer (2000), have demonstrated that psychological characteristics of others besides the leader often come into play. Specifically, these studies found that aggregation effects are found in public sources, such as speeches and press conference statements.

Operational code analysts utilize the following logic to aggregate a state's prevailing set of cognitions. According to Lake and Powell (1999), a state's actions are the outcomes of bargaining and strategic interaction processes inside the state. This is between players with preferences about different decisions on the menu for choice, which aggregate as outcomes to become a state's foreign policy decisions. Put more simply, a state's foreign policy decision 
occurs after the actors' involved in the decision making process have assessed the situation at hand and proceeded to take action (Schafer 2000, 513). This process forces those involved to compromise via bargaining and strategic interaction given that they are likely to have differing viewpoints or positions regarding the issue at hand. State action can only occur when they reach a collective decision, albeit a compromised one. It is this compromised decision that reflects the core psychological characteristics of those involved in the decision making process. When forced to compromise, an actor adheres to their core cognitions since they represent their underlying assumptions about the issue at hand (Beck 1995; Jervis 1976; Nisbett and Ross 1980).

Based upon this logic, operational code analysts employ the following procedure when determining a state's aggregated operational code. One needs to simply look at official statements of the administration to discern a state's operational code (Schafer 2000, 513). This is because they have been derived after the actors' involved have assessed the situation and proceeded to take action. That is, although there may be burueacartic wrangling over terms and positions, formal speeches are generally the state's decision-making apparatus and therefore represent the official views of the state (Schafer and Walker 2006, 47). This shared view of the state's foreign policy elite can be called "cognitions of the state," as reflected most clearly in the formal, prepared statements of the chief of state (Hermann 1985).

Due to aggregation effects, the operational code construct can be used as a group measure (Walker and Schafer 2000). It can be used to aggregate the cognitions found among members and groups in organizations, institutions, societies, and so on. In turn, operational code analysis can be used to study the core beliefs that the IC holds about world politics. Indeed, if we are able use OCA to aggregate the cognitions of the state, which is a collection of competing agencies 
and organizations, we can also use it to aggregate the beliefs of an entity with a similar structure, such as the IC. We are able to discern the worldview of U.S. intelligence by simply looking at public sources that reflect and aggregate the core beliefs that the various IC agencies hold about world politics.

In conclusion, this dissertation conceptualizes the IC's worldview in the form of an operational code, specifically as the five philosophical beliefs within it. This is due to four reasons. Firstly, it is a valid and longstanding tool in FPA for studying worldviews. Secondly, it is one of the most widely used and general set of cognitions. Thirdly, theoretically speaking, constructivism and the operational code construct are a good match. Finally, it can be used to aggregate the core cognitions that the various IC agencies hold about world politics with reasonable accuracy. This is done by examining the language in formal, prepared statements found within public sources that reflect what the various IC agencies collectively believe about foreign affairs and global issues among state and non-state actors. Chapter four of this dissertation discusses the various types of speech acts that be used to aggregate the fundamental beliefs that the intelligence agencies' hold about world politics.

\section{Chapter Summary}

This chapter explained how this dissertation conceptualizes the worldview of U.S. intelligence. It began by identifying four theoretical frameworks within the IR subfield of foreign policy analysis that could potentially provide the necessary theoretical framework to conceptualize the IC's fundamental belief system about world politics. I argued that although each has its advantages, the best way to conceptualize the worldview of U.S. intelligence is in the form of an operational construct, specifically as the five philosophical beliefs within it. This is because the operational code construct is a valid and longstanding analytical device that 
researchers and analysts use to assess a political actor's worldview regarding world politics, it matches well with the theory of constructivism, and OCA is the only analytical tool that has developed a procedure that can be used to aggregate the core beliefs that the various IC agencies hold about world politics. Therefore, the following five cognitions make up the IC's core belief system about world politics: the nature of the political universe, the prospects for realizing fundamental political values, the predictability of others in the political universe, control over historical development, and the role of chance. I define and discuss the logic behind each of these cognitions in the next chapter, which also presents this dissertation's theory and hypotheses. 


\section{CHAPTER THREE}

\section{THEORY AND HYPOTHESES}

This chapter presents this dissertation's theory and hypotheses. A theory is a set of related propositions that explain, and sometimes predict, why events occur in the manner that they do (Hoover and Donovan 2001, 33). Like concepts, theories are an essential element of social scientific thinking. They provide patterns for the interpretation of data, they link one study with another, they supply frameworks within which concepts and variables acquire substantive significance, and they allow us to interpret the larger meaning of our findings for ourselves and others (Ibid., 35). Hypotheses, which propose relationships between two or more variables, are equally important elements of social scientific thinking (Ibid., 27). For example, political participation increases with education. This simple assertion or educated guess based upon observation can be seen as a hypothesis because it has a subject (the variable, political participation), a connective verb (a relationship, increases), and an object (the variable, education).

Theories and hypotheses cannot be divorced from one another. A theory is a collection of hypotheses linked by some kind of logical framework (Ibid., 65). In other words, a theory is an explanation of a set of related observations or events based upon hypotheses. Hypotheses are tested by the researcher to assess the validity or accuracy of theory. This is extremely important given that theories are tentative formulations. In turn, this chapter does two things. First, it posits a theory. It explains where the cognitions the make up the IC's worldview are likely to be derived from. Second, this chapter presents the propositions or hypotheses that flow naturally from my theory and organize my study. These hypotheses will then be tested later on in this dissertation to gauge the accuracy of my theory. 


\section{Theory}

Where do the cognitions that make up the IC's worldview originate? One thing that cognitivists widely agree upon is that regardless of whether we are talking about individuals, groups, societies, or another entity, beliefs about world politics are not merely endogenous variables (Walker and Schafer 2006, 30). This is because "beliefs are subjective representations of reality" (Tetlock 1998, 876). They are simplified representations of the complex and ambiguous international scene that entities use to interpret events and choose among courses of action (Axelrod 1976a; Cottam 1986; Jervis 1976; George 1969, 1980; Vertzberger 1990). An actor may act rationally, but only within the context of their simplified subjective representations of reality. Because beliefs about world politics are not endogenously defined variables, I cannot simply assume that the IC's worldview derives from the role that intelligence plays in the U.S. national security framework, or from the intelligence agencies' bureaucratic interests and preferences.

Beyond this, however, very little empirical knowledge exists about where political worldviews originate (Koltko-Rivera 2004). This is due to the fact that "[w]hile this belief system in principle can be reduced to the information stored in complex systems of beliefs, feelings, and motivations, we cannot yet, in practice, trace it to this level of existence" (Walker and Schafer 2006a, 29). Instead, researchers generally access the contents and relationships of a belief system through its effects (Ibid., 29). Given that determining the actual causes of the IC's worldview is beyond the scope of this dissertation, I will simply deduce propositions about what the IC believes about world politics from several U.S. intelligence norms. As I explained in chapter one, the cognitions that the IC holds about world politics are partly responsible for shaping its identity. Its identity is also meant to help satisfy these beliefs. This suggests that 
U.S. intelligence norms are likely to derive, in part, from the core beliefs that U.S. intelligence holds about world politics. Therefore, the theory of this dissertation is that the core beliefs that the IC holds about world politics (the worldview of U.S. intelligence) underlie several U.S. intelligence norms. ${ }^{1}$

\section{Hypotheses}

Chapter two explained that this dissertation conceptualizes the worldview of U.S. intelligence in the form of an operational code, specifically as the five philosophical beliefs within it. Therefore, the following five cognitions characterize the IC's core belief system about world politics: the nature of the political universe, the prospects for realizing fundamental political values, the predictability of others in the political universe, control over historical development, and the role of chance. In this section, I briefly explain the logic behind each of these five cognitions and I hypothesize about how U.S. intelligence is likely to view each philosophical belief. Each hypothesis flows naturally from my theory. Evidence supporting my hypotheses is derived mostly from the work of constructivists writing about intelligence (Davies 2004; Sims 2005; Turner 1995, 2004, 2006; Wark 2003). I also utilize primary and secondary data to increase the credibility of my propositions. However, prior to hypothesizing about each philosophical belief, I need to discuss the continuity of the IC's core belief system about world politics.

\section{Continuity of the IC's Worldview}

In chapter one, I defined the worldview of U.S. intelligence as the set of core cognitions that the IC holds about foreign affairs and global issues among state and non-state actors (world politics). Core cognitions in this context refer to the most fundamental beliefs that the IC

\footnotetext{
${ }^{1}$ I state that the IC's worldview only underlies several, not all, intelligence norms since some of the norms, such as institutional survival, are not relevant to understanding cognition.
} 
generally holds about world politics. I emphasize the word "generally" because it symbolizes the common assumption made by researchers of belief systems that core beliefs are absolutistic and rigid (Beck 1995; Jervis 1976; Nisbett and Ross 1980). This suggests that the IC's core cognitions about world politics are specific and longstanding. On the other hand, operational code analysts have long debated the temporal stability of the five philosophical beliefs in an operational code.

Early studies on the operational code generated hypotheses that operational code beliefs were internally coherent, remained stable over time, and extended across issues domains for a particular leader. These hypotheses were derived from the fact that the operational code was originally conceptualized as a "configuration of ideas and attitudes in which elements are bound together by some form of constraint or functional interdependence" (Converse 1964, 207-208). However, these propositions were challenged by Alexander George $(1969,1979)$ and Ole Holsti $(1977,1979)$. These individuals posited that not all political actors have a single, well defined set of operational code beliefs. Leaders may change their beliefs over time. Subsequent research in the 1980s, 1990s, and 2000s validated this forecast. The results from several studies indicated that a political actor's operational code beliefs are likely to vary in degree over time and for different issue areas in the political universe (Crichlow 1998; Marfleet 2000; Schafer and Crichlow 2000; Walker, Schafer, and Young 1998, 1999; Walker and Falkowski 1984). An equally important finding that emanates from these studies is that the philosophical beliefs in an operational code are especially prone to fluctuation by domain over time in responses to changes in context (Schafer and Crichlow 2000; Walker, Schafer, and Young 1998). This stance is consistent with the literature on complex and diagnostic learning (Malici and Malici 2005). ${ }^{2}$

\footnotetext{
${ }^{2}$ Complex learning occurs when an actor's key philosophical beliefs about political goals and key instrumental beliefs about the most effective means to achieve them are modified so as to alter strategic preferences, whereas
} 
These research findings bring into question whether or not the IC's worldview is specific and longstanding. Has the worldview of U.S. intelligence existed in a singular form for a long period of time, or has it changed over time in responses to changes in context? According to constructivists writing about intelligence (Davies 2004; Sims 2005; Turner 2004, 2006; Wark 2003), intelligence norms are the product of the dominant U.S. political culture and its subset, U.S. strategic values. These fairly specific and longstanding values transcend partisan politics and particular administrations. For example, "the perceived right to privacy, while not actually set forth in the U.S. Constitution, is an enduring norm of American political life" (Sims 2005, 34). The central point is that although not all American are of the same mind all of the time, certain central themes in the U.S. cultural tradition recur repeatedly. This provides patterns of thought and action in national security matters (Jordan, Taylor, and Mazarr 1999, 48).

Constructivists writing about intelligence delineate the norms of U.S. intelligence by utilizing the typology of America's strategic culture produced by Donald Snow (1995). Snow posits that three important influences shape how Americans view the role of defense and national security: America's lack of a sense of history, the unique American geographical endowment, and the nation's Anglo-Saxon heritage. These three influences give rise to seven factors that make up America's strategic culture: America's democratic institutions, the role of the media on strategic matters, and underlying authoritarianism and suspicion of things related to government, a belief in the efficacy of the citizen soldier, the belief in American invincibility, the tradition of mobilization and demobilization, and ambiguity about the nature of war. Constructivists writing about intelligence argue that the U.S. national security establishment has internalized this strategic culture and transformed it into mission specific norms that shape policymaker preferences regarding intelligence. This leads to the norms that make up the U.S. intelligence 
identity.

Even though students of intelligence like to point out that the United States does not have much of an intelligence history, they do note that "intelligence has been a function of the [U.S.] government since the founding of the republic. While it has had various incarnations over time, intelligence has historically played a key role in providing support to U.S. military forces and in shaping the policies of the United States towards other countries" (Johnson and Wirtz, eds. 2004, 5). Moreover, although various agencies have been added to the IC over the years, its basic structure and culture have been remarkably stable since its establishment in the National Security Act of 1947 (Lowenthal 2006, 30; Turner 2006, 43). This is due, in part, to U.S. intelligence norms (Turner 2006, 43). Because U.S. intelligence norms are partly products of the dominant U.S. strategic culture and its subset, U.S. strategic values, the core beliefs about world politics that underlie it are likely to be similarly specific and longstanding.

Additionally, studies that question the temporal stability of the five philosophical beliefs in an operational code are talking about individuals, not organizations such as the IC. It is difficult for an organization's culture, and the belief system that underlies it, to change because they are rooted in the collective history of an organization (Cameron and Quinn 1999; Hatch 2004; Schein 1996, 2004). This is not to say that an organization's culture and core cognitions cannot change. Numerous studies present strategies to do just this. Instead, it indicates that organizational cultures and beliefs are generally specific and longstanding, and only change when radical change in an organization occurs. Given that the basic culture and structure of the IC's has been very stable since 1947, it is unlikely that the intelligence agencies' core belief system about world politics has changed much, if at all, over the years.

In conclusion, despite research by cognitivists indicating otherwise, the preceding 
discussion suggests that the IC's worldview has existed in a singular form for a long period of time. This leads to the following hypothesis regarding the continuity of the IC's worldview: The worldview of U.S. intelligence is specific and longstanding. Remember, I am referring to the core cognitions that the IC holds about "others" in the political universe, and not the core cognitions that it holds about "self." I define the worldview of U.S. intelligence as the set of core cognitions that the IC holds about foreign affairs and global issues among state and nonstate actors. I am not arguing that the IC's instrumental beliefs about world politics are specific and longstanding as well.

\section{The Nature of the Political Universe}

The fundamental nature of politics, political conflict, and the image of the opponent is the first philosophical belief in an operational code. This captures what the essential nature of political life is. It informs us whether the political actor thinks the political universe is fundamentally cooperative, conflictual, or on a continuum between these two poles. This is one of the most critical beliefs in an operational code. Indeed, "a political actor's belief system about the nature of political life is shaped particularly by [their] political orientation to other political actors. Most important of these are one's opponents. The way in which they are perceived exercises a subtle influence on many other philosophical and instrumental beliefs in an operational code" (George 1969, 201-202).

According to Turner (2004), "much of the task of intelligence takes place in secrecy, an issue that surrounds and sets parameters around intelligence activities. Moreover, secrecy—and the classification scheme that sets the basis for it - provides the cement for the U.S. Intelligence Community and beyond" (49). Secrecy became an extremely important norm within the IC during the Cold War because working against the Soviet Union, a particularly secretive state, 
was its highest priority. "From the late 1940 s to the late 1980 s, the chief test of the quality of U.S. intelligence agencies was their capacity to gauge the capabilities and intentions of the Soviet Union..." (May 2005, 3). This caused secret sources of information to be given more value and importance than their open source counterparts (Sims 2005, 37). At the same time, the constant spy wars between the CIA and the Soviet Union's Committee for State Security (KGB) made the need for secrecy increasingly necessary to protect sources and methods. For example, in 1985, U.S. law enforcement identified and/or arrested several high profile foreign spies working outside or within the IC. This led to increased security and protection against infiltration, thereby buttressing the importance for secrecy.

After the end of the Cold War, there were calls to downsize the national security apparatus in the U.S. government, including the intelligence agencies', given that the Soviet Union no longer existed. However, by the end of the 1990s, new and intractable security threats, such as terrorists and narcotics traffickers, began to emerge. This helped thrust the IC back into the center of U.S. national security policy, and once again buttressed the need for secrecy. This was due to not only the legacy of the Cold War and new and intractable security threats, but also because U.S. intelligence agencies could not, and cannot, restrict themselves to only thinking about enemies. They must also keep track of powers that are neutrals, friends, and even allies. Neutrals, friends, and allies can be rivals in certain contexts (Lowenthal 2006, 5). For instance, even though the European Union and Japan are U.S. allies, they compete with the U.S. for global resources and economic markets. Furthermore, although the U.S. and Russia ostensibly became friends during the 1990s, FBI agent Robert Hanssen, who began spying for the Soviet Union in 1979, continued his work as a spy for Russia until his capture in 2001. Other notable examples include the cases of Harold James Nicholson, who was a senior CIA officer that spied for Russia 
during the mid-1990s, and Aldrich Ames, who spied for the Soviet Union and Russia from 1985 to 1994.

It is safe to say that the norm of secrecy is one of the most important of the IC's norms (Sims 2005; Turner 2004). In fact, it is so entrenched in America's intelligence history that its origin can be traced back to the days of George Washington. Washington repeatedly insisted that the secrecy of intelligence operations was a condition of their success: "The necessity of procuring good intelligence is apparent and need not be further urged-All that remains for me to add is, that you the whole matter as secret as possible. For upon Secrecy, success depends in most Enterprizes of the kind, and for want of it, they are generally defeated, however, wellplanned..." (Andrew 1995, 8).

The norm of secrecy implies that the IC is likely to believe that the political world is fundamentally conflictual. This suggestion is strengthened by the role that a threat based foreign policy has played in the development of the U.S. intelligence system (Lowenthal 2006, 14). A major reason why the IC exists in the first place is to help shield the U.S. from external threats to its vital security interests (Vital 2003). The twentieth century saw the repeated rise of powers whose foreign policies were direct threats to the international status quo, which the U.S. assumed a vested interested in with the promulgation of the Monroe Doctrine in 1823 (Lowenthal 2006, 13). There was Kaiserine Germany in World War I, the Axis in World War II, and the Soviet Union. It is threats such as these that prompted the formal establishment of the IC in 1947 (Ibid., 13). Intelligence operations were one way in which the U.S. could counter these threats. These types of threats serve as the primary reasons why the IC continues to exist to this day. "The terrorism threat in the late twentieth and early twenty-first centuries fits the same pattern of an opponent who rejects the international status quo" (Ibid., 13). 
The norm of secrecy and the role that a threat based foreign policy has played in the development of the IC indicates that U.S. intelligence views "others" in the political universe as generally being hostile to the U.S. and by extension the IC. This is buttressed by that the fact that intelligence, in general, is essentially about reducing uncertainty in conflict (Clark 2004, 13). Granted, the U.S. does have friends and allies. However, friends and allies can be enemies in certain contexts. As the proverb goes, keep your enemies close but your friends closer. All of this leads me to hypothesize the following about what the IC believes about the nature of the political universe: U.S. intelligence has a hostile image of the political universe and the fundamental nature of their political opponents.

\section{The Prospects for Realizing Fundamental Political Values}

The general prospect for realizing one's fundamental political values is the second philosophical belief in an operational code. This cognition represents how likely a political actor believes it is that they will achieve their basic political goals. Can one can be optimistic, or must one be pessimistic on this score? According to Schafer and Walker (2006, 33), an actor that sees a positive, friendly world is likely to be more optimistic about realizing their fundamental political values. One who sees a hostile world of other actors trying to block the realization of their objectives is likely to be more pessimistic. However, as George (1969) first pointed out, an optimistic view can be tinged with conditional pessimism and vice-versa. Although the conventional Bolshevik was optimistic, the Soviets also believed that catastrophe could not be excluded and was an ever-present danger (Ibid., 203). Therefore, similar to the first philosophical belief, a political actor can be optimistic about realizing their fundamental political values, pessimistic, or on a continuum between these two poles.

Given that I have already hypothesized that the IC has a hostile image of the political 
universe and the fundamental nature of their political opponents, logic suggests that U.S. intelligence should be pessimistic regarding the realization of its ability to shield the U.S. from external threats to its vital security interests. This is not irrational thinking due to the fact that the U.S. has many enemies and there are instances where friends and allies can become opponents. Furthermore, every intelligence community in the world has a counterintelligence component. Counterintelligence, in this context, refers to efforts taken to protect one's own intelligence operations from penetration and disruption by other nations intelligence service (Lowenthal 2006, 154).

Nevertheless, careful study of U.S. intelligence norms indicates that the IC's belief about the realization of its fundamental political values goes against conventional wisdom. There is a "can do" attitude in the IC that mirrors its cousin in the strategic culture (Turner 2004, 55). This view, that American intelligence can accomplish any task successfully, is a reflection of the broader American principle that Americans are doers, that they can do any task set before them better than anyone else (Snow 1995, 24-29). ${ }^{3}$ This attitude also derives from the essential optimism of Americans, based on the assumption that daring and persistence are the attributes most likely to yield useful intelligence about national security threats (Haley 1989, 496). These assumptions are essential to the IC's esprit de corps. ${ }^{4}$ Since the IC's inception, the intelligence agencies' have exhibited an optimism that operates on the assumption that imagination, daring, and persistence are the attributes most likely to yield the most useful intelligence about national security threats (Turner 2004, 55). For example, the objectives outlined in a 1999 speech are illustrative of a "can do" attitude that has prevailed in the IC since its inception (Ibid., 55).

\footnotetext{
${ }^{3}$ This principle derives from the longstanding belief within American culture that the U.S. differs qualitatively from other developed nations due to its national credo, historical evolution, and its distinctive religious and political institutions.

${ }^{4}$ Esprit de corps is a French phrase meaning "spirit of a body."
} 
The norm of intelligence exceptionalism strengthens the optimism that the IC's "can do" attitude breeds. This norm refers to the widespread belief in the IC the function it performs is different from and more important than other government activities (Sims 2005; Turner 2004, 2006). Indeed, the IC believes that it is the only entity capable of making sure that policymakers are well-informed about the state of the world and the likely consequences of various policies and actions (Richelson 2008, 1).The norm of intelligence exceptionalism arises from several factors. According to Turner (2004),

First, intelligence is different because it employs secrecy as a guiding principle. Second, intelligence is different because it entails, among other things, the breaking of other countries' laws. Third, intelligence is different because it is subject to deception and disinformation, often casting doubt on the reliability and integrity of information - the principal product of intelligence activities. Fourth, intelligence is different because it is fungible; that is, it can be, and often is, used by leaders and politicians for a variety of purposes, ranging from furthering the public interest to promoting a partisan or private agenda. (50)

The norm of flexible accountability furthers the intelligence agencies' belief that they are exceptional among U.S. government agencies and organizations. This is because the IC accounts for its actions and performance differently from other government institutions (Ibid., 49).

In conclusion, the IC's "can do" attitude makes it unlikely that the IC has a pessimistic view regarding the realization of its fundamental values. This is not to say that U.S. intelligence has never doubted its ability to achieve its basic political goals. Since the 1970s, the IC has often skirted risk to avoid running afoul of U.S. law or becoming subject of political fallout. Still, the IC firmly believes that it is the only entity within the U.S. government that is well-suited enough to yield useful intelligence about national security threats. The norms of intelligence exceptionalism and flexible accountability act as buffers against any doubt in the IC regarding its ability to realize its fundamental political values. Therefore, contrary to conventional wisdom, 
the IC's "can do" attitude leads to the following hypothesis: U.S. intelligence has an optimistic view regarding the realization of its fundamental political values.

\section{The Predictability of Others in the Political Universe}

The third philosophical belief in an operational code is the extent to which the political actor believes the political future is predictable. This belief is about the stability of the course of future political events. Is the world in constant change, or do world events occur in a predictable fashion? Although this cognition is not necessarily an assessment of reality, which is true for all of the ten beliefs that comprise an operational code, it reflects the political actor's underlying belief about predictability. According to Schafer and Walker (2006a), "an actor could live in a world where others are pursuing a full variety of cooperative and conflictual actions, but if the actor only perceives others as pursuing threats and punishments, then he believes others' next moves are more predictable" (34).

Collecting intelligence information is of little value unless someone corroborates and evaluates the information, puts it into context, and uses it to form serious judgments about foreign capabilities and intentions (Turner 2006, 107). In turn, a distinguishing norm of U.S. intelligence is the importance it places on the production of high quality intelligence analysis (Turner 2004, 56). No government devotes as much time, money, and energy to current and long-term intelligence analysis (Ibid. 56). ${ }^{5}$ Moreover, despite all the attention that the operational side of intelligence receives (collection and convert action), analysis is the mainstay of the intelligence process (Lowenthal 2006, 108). As former Director of Central Intelligence (DCI) Richard Helms (1966-1973) asserts, “[c]asting aside the perceived—and I must admit the

\footnotetext{
${ }^{5}$ Current intelligence refers to intelligence that is processed and analyzed on daily or ongoing international matters. On the other hand, long-term intelligence refers to in depth research projects on a region, country, or issue that may not necessarily have current importance but may contribute to understanding an intelligence problems or developing future policy options.
} 
occasionally real-excitement of secret operations, the absolute essence of the intelligence profession [in the U.S.] rest in the production of current intelligence reports, memoranda and National Estimates on which sound policy decisions can be made" (Helms, Hood, and Kissinger 2003).

The primacy that the IC places on high quality intelligence analysis can be traced back to World War II. ${ }^{6}$ According to Woodrow Kuhns (2007),

[d]uring World War II, the United States made one of its few original contributions to the craft of intelligence: the invention of multisource, nondepartmental analysis. The Research and Analysis (R\&A) Branch of the Office of Strategic Services (OSS) assembled a talented cadre of analysts and experts to comb through publications and intelligence reports for clues to the capabilities and intentions of the Axis powers. R\&A's contributions to the war effort impressed even the harshest critics of the soon-tobe dismantled OSS. President Truman paid implicit tribute to R\&A in late 1945 when he directed that it be transplanted into the State Department at a time when most of OSS was being demobilized. The transplant failed, however, and the independent analytical capability patiently constructed during the war had all but vanished when Truman moved to reorganize the nation's peacetime intelligence establishment at the beginning of 1946 .

Eventually, in early February, the Office of Reports and Estimates (ORE) was created under the interagency Central Intelligence Group to perform intelligence research, produce current intelligence, and write National Intelligence Estimates for policymakers. This led to the first daily intelligence report, known as The Daily Summary. After the National Security Act of 1947 was passed, the ORE was integrated into the newly created CIA thereby making it the first analytical component of the newly created IC. ${ }^{7}$

\footnotetext{
${ }^{6}$ Before the U.S. entered World War II, American policymakers relied heavily on friends traveling abroad to provide them with informal intelligence analyses on the intentions of other leaders and other international developments (Aspin-Brown Commission 2004). Moreover, prior to the end of World War II, intelligence analysts focused mostly on understanding the capabilities and structure of foreign military organizations, as well as on deciphering and interpreting formal military communications (Ibid.). This was due to the fact that foreign intelligence was largely the purview of military agencies, such as Office of Naval Intelligence and the Army Signals Corps.

${ }^{7}$ The ORE was abolished in November 1950 and was replaced by three new units: the Office of National Estimates, the Office of Research and Reports, and the Office of Current Intelligence. These steps finally ended the confusion over the analytical mission, primarily by splitting the competing functions of national, current, and basic intelligence into three offices (Kuhns 2007).
} 
Ever since the creation of the ORE, high quality intelligence analysis has been at the heart of the U.S. intelligence process. The norm of competitive intelligence, which is a hallmark of U.S. intelligence analysis, reinforces this. Competitive intelligence-which refers to competition among IC agencies to collect, analyze, and produce information to increase the robustness of finished intelligence products - occupies a privileged position in the conduct of American intelligence (Turner 2006, 48). Many policymakers and scholars assert that the system of competition among intelligence agencies allows for "the truth to come out" (Turner 2004, 51). Each IC agency attempts to maximize its own position via "one-upmanship," which enables the acquisition of intelligence that other agencies may have missed. This gives maximum benefits to the intelligence consumer and even the American public. Thus, it is not surprising that competitive intelligence has accounted for several of the ICs intelligence successes over the years (Turner 2006, 48).

The IC's heavy focus on current and long-term intelligence, as well as the privileged position of competitive intelligence analysis, causes the IC to believe that it can understand and forecast world events with reasonable accuracy. That is, because of the high quality intelligence analysis it produces, the IC believes that world events do occur in a predictable fashion. Granted, the IC has experienced many intelligence failures throughout its relatively short but storied history. Also, in recent years, current intelligence has been given preference over longterm intelligence. This has been bolstered by the shift from enduring targets to fluctuating targets in the current era (Betts 2002). However, the longstanding primacy of high quality intelligence analysis demonstrates a "can do" attitude about understanding the political future. This leads to my fourth hypothesis, which is that U.S. intelligence believes that the political future is predictable. 


\section{Control over Historical Development}

The fourth philosophical belief in an operational code is the extent to which a political actor believes that they can influence historical development and control outcomes. How much control or mastery does the subject believe they have over historical development, specifically political events? The answer to this question emphasizes the role that dedicated, disciplined, and intelligent political actors can play in "moving" history in the desired direction (George 1969, 204). Seizing and utilizing every opportunity available is essential to alter the historical development of political events. The ability to control historical development within an operational code concerns itself with the "self" and "other."

There is no doubt that intelligence plays a vital role in shaping U.S. foreign policy. The IC has on many occasions greatly influenced foreign policy outputs. Intelligence changed Ronald Reagan's policy toward the "Evil Empire," and strongly influenced President George H.W. Bush's decisions regarding Desert Storm (Andrew 1995). Furthermore, covert operations conducted by IC agencies, ${ }^{8}$ especially as the CIA, have greatly influenced political events around the world. Covert operations are "designed to influence foreign governments, events, organizations, or persons in support of United States foreign policy" (Goodman 1987, 130). One example of this is the 1953 Iranian coup d'etat, which saw the overthrow of the democraticallyelected administration of Iranian Prime Minister Mohammed Mosaddeq and his cabinet from power by British and American intelligence operatives working together with Iranian agents and elements of the Iranian army. Many believe that the coup made the 1979 Islamic revolution in

\footnotetext{
${ }^{8}$ A covert operation or action is a secret government program in pursuit of foreign policy objectives by influencing events abroad in ways unattributable to the U.S. government (Turner 2005, 44). The National Security Act of 1947 made covert action an essential part of the American intelligence repertoire by granting the National Security Council (NSC) the authority to direct "special activities" from time to time. Various successes in the 1950s pushed covert action into the forefront of U.S. intelligence methods. Although several failed covert actions in the 1960s led to restrictions on the conduct of covert operations, covert action remains a major part of the IC's toolbox, especially in the current War on Terror.
} 
Iran inevitable (Kinzer 2003). There are several other examples of how covert action by U.S. intelligence influenced world politics. The most salient of these include the Bay of Pigs invasion in $1961,{ }^{9}$ covert action in Chile from 1963 to $1973,{ }^{10}$ and the Iran-Contra Affair during the 1980s. ${ }^{11}$

What the preceding paragraph suggests is that the IC has, in the past, greatly influenced historical development. This does not necessarily mean, however, that it believes it has a high degree of control over political events. The strict separation of intelligence from policy indicates otherwise. The strict separation of intelligence from policy is almost a truism in the intelligence business. According to Turner (2006), "[t]o intelligence officials this is an insurmountable principle, on the grounds that intelligence officers must maintain objectivity and keep clear of policy advocacy. Intelligence analysts are admonished to present their intelligence data to policymakers without bias or political taint" (50). To do so threatens the objectivity of the analyses they present (Lowenthal 1999, 3). Policymakers also hate to hear bad news. Bad news in this context refers to intelligence that might contradict or undermine organizational interests or preconceived policy. This is another strong impulse for intelligence and policy to stay apart.

Even though intelligence is irrelevant without policy and policy is blind without intelligence (Macartney 1988), the IC only informs the policy process by providing intelligence.

\footnotetext{
${ }^{9}$ The Bay of Pigs Invasion was an unsuccessful attempt by a U.S. trained force of Cuban exiles to invade south-west Cuba and overthrow the Cuban government of Fidel Castro. After the Cuban military defeated the invading force in a matter of days, the event accelerated a rapid deterioration in Cuban-American relations, which was further worsened by the Cuban Missile Crisis the following year.

${ }^{10}$ U.S. intelligence, specifically the CIA, United States ran covert actions in Chile between 1963 and 1973 leading to the ascension of General Pinochet. The CIA helped Pinochet topple the government of President Salvador Allende to prevent the emergence of socialism in Chile. However, after General Pinochet formed a military junta, he moved to solidify his control against any opposition, which led to the Caravan of Death (Caravana de la Muerte) and Operation Condor. The former was a Chilean Army death squad whose members ordered or personally carried out the execution of at least 75 individuals held in Army custody, while the latter was a campaign of political repressions involving assassination and intelligence operations officially implemented in 1975 by the right-wing dictatorships of the Southern Cone of South America.

${ }^{11}$ The Iran-Contra affair was a covert operation that provided funds to the Nicaraguan contra rebels from profits gained by selling arms to Iran.
} 
It does not make foreign policy decisions. The locus-of-control lies with the policymaker. ${ }^{12}$ As Turner (2004) notes, "nothing prevents a policymaker from rejecting intelligence analyses or providing intelligence outputs of his own" (55). Furthermore, all the actions of the IC are either explicitly or implicitly directed by the president and his or her senior policymakers (Powers 2004). Christopher Andrew's book For the President's Eyes Only (1995) does an outstanding job at detailing how U.S. presidents have used secret intelligence since the founding of the nation.

Since foreign policymakers are the ones who are most often interacting with external actors' that have the ability to greatly influence historical development, such as nation-states and international organizations, the IC's ability to influence world political events is further reduced. This is not to say that the IC does not interact with important external actors. U.S. intelligence works to infiltrate and conduct intelligence operations against foreign intelligence organizations, terrorist groups, and other important groups, institutions, and organizations at the state and nonstate levels (Lowenthal 2006). Rather, foreign policymakers, especially the president, are the ones interacting constantly with those who have historically made decisions that altered historical development. Would there have been a nuclear exchange if someone other than Khrushchev been head of the Soviet Union during the Cuban Missile Crisis? Would World War II have happened if it were not for Hitler? Would the Second Persian Gulf War have happened if someone other than Saddam Hussein had been the president of Iraq? What counterfactuals such as these suggest is that the locus-of-control for influencing historical development lies not only

\footnotetext{
${ }^{12}$ Within psychology, locus-of-control is considered to be an important aspect of personality. Locus-of-Control refers to an actor's perception about the underlying main causes of events in his/her life. Or, more simply, does one believe that their destiny is controlled by oneself or by external forces (such as fate, god, or powerful others)? See Kaplowitz (1978), Langer (1983), Lefcourt (1976), and Phares (1976) for further discussion.
} 
with U.S. foreign policymakers, but also with important external actors ("others") who the IC only sporadically deals with.

In the end, despite the fact that IC has on many occasions greatly influenced foreign policy outputs, the strict separation of intelligence from policy indicates that U.S. intelligence believes that it has a low degree of control over historical development. U.S. intelligence does not believe it has much control or mastery over world political events. This is buttressed by the fact that the locus-of-control regarding foreign policy decisions lies with foreign policymakers and other important external actors. It lies with "others" and not "self." This discussion leads to my fifth hypothesis, which is that U.S. intelligence believes that it has a low degree of control over historical development.

\section{The Role of Chance}

The role of chance is the fifth and final philosophical belief in an operational code. This characteristic represents the degree to which political actors believe that the world around them is the product of chance. What is the role of chance in human affairs and in historical development? This characteristic is largely a function of the third philosophical belief about the predictability of the political future (Crichlow 2005, 191). Indeed, if a political actor believes that the political future is predictable, for example, logic dictates that they also believe that the role of chance is low. This cognition is also related to what the actor believes about control over historical development (Schafer and Walker 2006a, 34-35). However, the impact of the fourth philosophical belief is likely to be minimal with regard to the IC given the norms of policy support and "accurate, timely, and relevant intelligence." The former often spills over into policy advocacy (Turner 2006, 55). The latter reduces uncertainty thereby enabling political and military leaders to improve the quality of their decisions, develop more effective strategies, and 
conduct operations that are more successful (Handel 1988, 3). Therefore, given that I have already hypothesized that U.S. intelligence believes that the political future is predictable, my sixth hypothesis is that U.S. intelligence does not attribute a great role to chance.

\section{Chapter Summary}

This chapter began by formally presenting this dissertation's theory, which was made implicit in chapter one. The theory states that because U.S. intelligence norms derive, in part, from the IC's worldview, then the worldview of U.S. intelligence must underlie several U.S. intelligence norms. The chapter then presented six hypotheses. The first states that the worldview of U.S. intelligence is specific and longstanding. This proposition mirrors the constructivists' argument that the U.S. intelligence identity is well-established and continuous. The other five propositions focus on the philosophical beliefs in an operational code. Interestingly, these five hypotheses reflect the tension between and among some of the intelligence norms. For example, although I hypothesized that the IC believes the world is full of hostile actors, I also proposed that the IC is optimistic about realizing its fundamental political values. Tensions such as these probably reflect the uncertainty that the IC has about what role it ought to play in United States society (Turner 2004, 57). 


\section{CHAPTER FOUR}

\section{METHODOLOGY AND DATA}

In the previous chapter, I presented this dissertation's theory and posited six hypotheses. The first hypothesis focused on the continuity of the IC's worldview and the other five propositions stated how U.S. intelligence is likely to view each philosophical belief within an operational code. However, until these hypotheses are tested against empirical data, they are simply tentative answers to my research question. This chapter explains what methodology and data this dissertation will use to confirm, or deny, their credibility. I describe my research design and method of data collection. The research design I present is quantitative rather than qualitative in nature. I have decided in favor of quantitative analysis given that it is predominant in contemporary operational code analysis, less subjective, and more conducive to analyzing my hypotheses over time.

This chapter is broken up into several sections. The first identifies and discusses in detail the systematic coding procedure that this dissertation employs to quantify the IC's five philosophical beliefs about world politics. I employ the Verbs in Context System (VICS) of content analysis. A discussion on the specific approach that will be used for VICS coding follows this section. This is necessary given that VICS coding can be done either by hand or with a computer. In the sections that follow, I identify my data source and sampling frame. I wind down the chapter with a discussion on how I will use the data garnered from VICS coding to statistically analyze my hypotheses. The chapter concludes with a summary of the preceding sections. 


\section{Quantifying the Worldview of U.S. Intelligence}

The methodological precedent for operational code analysis was set by Nathan Leites in his 1951 study on Bolshevik behavior (Young and Schafer 1998, 71). Indeed, several studies subsequent to Leites utilized his methodological approach (e.g., Johnson 1977; McLellan 1971; Walker 1977; Stuart and Starr 1981). In each of these projects, Alexander George's (1969) ten operational code questions served as the organizing framework. Researchers then reviewed the selected material for evidence that would provide answers to George's ten questions. This approach generated detailed and rich analyses of key political actors. However, Leites's approach suffered from many shortcomings (Young and Schafer 1998, 71). For one, this type of analysis was costly in terms of time. Moreover, determining the reliability of such analyses was difficult since it was virtually impossible to replicate. It was also quite hard to compare and contrast the results of the various operational code analyses that adopted this approach. Very few "thick" analyses were being done.

Shortcomings such as these led Ole Holsti (1977) to construct a coding manual composed of sets of items whose answers were intended to assess George's ten questions in a systematic manner. Holsti also suggested that the answers to the operational code questions should be organized into a typology. Holsti's coding manual and suggestion eventually became important contributions to operational code analysis literature. His work was important to the development of the way of assessing the operational code (Young and Schafer 1998, 72). Nevertheless, Hoslti's work received little follow up attention (Ibid., 72). One reason was that the coding questionnaire was long and cumbersome. Another was that despite organizing George's construct into a refined set of questions, researchers still needed to peruse and interpret texts to identify answers to the operational code questions. Thus, operational code analysis still had to 
be done through the subjective, interpretative review of texts.

The limits of Holsti's methodological construct led Stephen Walker and Lawrence Falkowski (1984) to turn Holsti's questionnaire into an inventory that could be filled out by others either associated with or studying particular political actors, especially leaders. This methodology proved to be much more expeditious than previous ones. Still, this approach had its limitations, which meant that operational code methodology was still subjective and unreliable. As Young and Schafer (1998) explain, "[t]he subjectivity of the analysis remains high but takes on a different form. Among the problems that arise are selective remembering, post hoc justifications, and respondents who do not normally think about the subject in operational code terms" (72).

This more or less remained the case until 1998 when Stephen Walker, Mark Schafer, and Michael Young introduced a content analysis coding scheme that focuses on the verb-based attributions of political actors to answer George's ten questions. Known as the Verbs in Context System or VICS, this approach has made operational code analysis less subjective, more reliable, and less labor intensive. It has since become the predominant methodology used by operational code analysts. It is for these reasons that this dissertation employs VICS as the systematic coding procedure that will quantify the IC's core beliefs about world politics.

\section{What is the VICS Method?}

The VICS method is similar to other political psychology methods that study leader characteristics "at a distance" (Schafer and Walker 2006b, 566-567). The "at a distance" research program draws on assumptions and methods found in psychology, political science, and speech. The core assumption of this research program is that a subject's verbal statements can be used to assess their psychological characteristics. For example, Hermann (1980) argues that 
an actor who uses a lot of complex words in their speech will be more conceptually complex. That is, they will see the world in more differentiated categories as opposed to simple black-andwhite terms. In addition to providing information on psychological characteristics, data gathered at a distance can be easily used in statistical models to compare and contrast political actors and to connect the traits with political variables of interest (Schafer 2000, 512). VICS focuses on the verbs in a political actor's public or private statements and their attributions regarding exercise of power to the "Self" and "Others" to construct quantitative indices that correspond to the philosophical and instrumental beliefs in an operational code.

The objective of VICS is to conduct content analysis of verbal material that can be used to assess the cognitive beliefs of political actors in the form of an "operational code" (Schafer and Walker 2006a, 30). The premise for this system is that the way actors speak about power relationships in the political universe will tell use much about their beliefs regarding their exercises of power. Therefore, VICS focuses primarily on verbs in speech acts by political actors rather than in secondary sources or reports by external observers such as historians or journalists. Speech acts include, but are not necessarily limited to, texts of public or private statements in the form of diaries, letters, speeches, memoranda, interviews, and press conferences. The data source that VICS uses is the speech act and the recording unit is the "utterance," which is each verb in the statement and the corresponding parts of speech associated with each verb - the subject and object (if it is a transitive verb) or the subject and predicate nominative or adjective (if it is it an intransitive verb). As Figure 4.1 illustrates, the VICS method extracts values for six attributes for each recording unit (verb) and its surrounding context: subject, verb category, domain of politics, tense of verb, intended target, and context. 


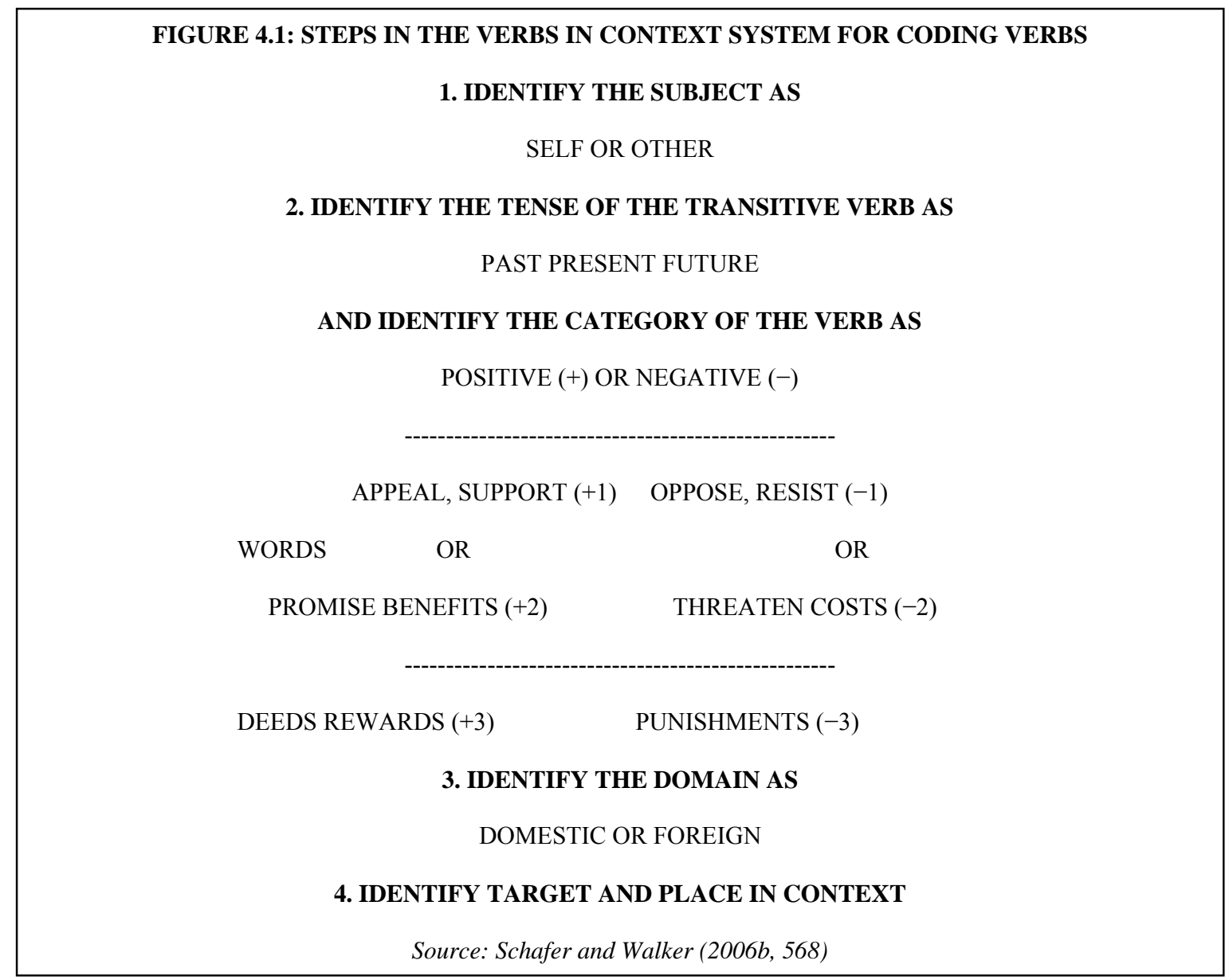

Self or Other designates whether the political actor under investigation or some other actor is the subject of the verb. VICS categorizes the verb in its tense either as a positive $(+)$ or negative (-) intransitive verb or a positive $(+)$ or negative $(-)$ transitive verb. If it is a transitive verb, it is categorized further as representing either a cooperative (+) or conflictual (-) behavior that takes the form of a word or a deed. ${ }^{1}$ Positive transitive deeds are coded as Rewards $(+3)$, while negative transitive deeds are coded as Punishments (-3). Positive transitive words are coded as either Promises $(+2)$ or Appeal/Support $(+1)$, whereas negative transitive verbs are coded as either Threats (-2) or Oppose/Resist (-1). Verbs that do not fit into one of these categories, or that do not have a political context, are coded as Neutral (0) and discarded. The

${ }^{1}$ Deeds are actions that have been done, while words are promises or threats of future action or symbolic declarations of support of opposition. 
remainder describes the political actor's beliefs about the intended or imagined exercise of power by self and others regarding the political issues that the speech act raises. ${ }^{2}$

Once the utterances are sorted and aggregated, the results provide the foundation of the VICS system - the quantitative answers to the ten operational code questions. When a researcher wants to know an actor's philosophical beliefs, he or she simply has to aggregate and index the verbs where the political actor is talking about other actors. On the other hand, when we are concerned with an actor's instrumental beliefs, the researcher indexes the verbs where the political actor is talking about self and their in-groups. When taken together, the researcher has a picture of a political actor's belief system regarding acts of conflict and cooperation by self and others in the political universe. He or she has their operational code.

\section{Calculating the Philosophical Indices}

The VICS system contains a total of seventeen different indices. All rest fundamentally on the logic of VICS content analysis discussed in the previous sub-section. However, I will only discuss the five philosophical indices in detail. As I explained in chapter two, the philosophical beliefs in an operational code, not the instrumental ones, comprise the IC's core belief system about world politics. A summary of how VICS calculates each philosophical index is provided in Figure 4.2. It is important to note that this discussion is based on Walker, Schafer, and Young (1998) and Schafer and Walker (2006). The former introduced VICS and the latter is the most recent summation of VICS coding.

The first philosophical index is the nature of the political universe (P-1). This index is purposefully broad and general so that the balance of the political actor's views of other actors in the political universe can be captured (Schafer and Walker 2006, 33). VICS assumes that the

\footnotetext{
${ }^{2}$ They are also the basis for drawing inferences about subsequent behavior by the political actor (Walker, Schafer, and Young 1999).
} 
political actor's images of other actors' policies and actions reflect one's beliefs about politics,

\begin{tabular}{|c|c|c|}
\hline \multicolumn{3}{|c|}{ FIGURE 4.2: CALCULATING THE PHILOSOPHICAL INDICES } \\
\hline $\begin{array}{l}\text { P-1. NATURE OF THE POLITICAL } \\
\text { UNIVERSE (Image of Others) }\end{array}$ & $\begin{array}{l}\text { \%Positive minus \%Negative } \\
\text { Transitive Other Attributions }\end{array}$ & $\begin{array}{l}+1.0 \text { friendly to } \\
-1.0 \text { hostile }\end{array}$ \\
\hline $\begin{array}{l}\text { P-2. REALIZATION OF POLITICAL } \\
\text { VALUES (Optimism/ Pessimism) }\end{array}$ & $\begin{array}{l}\text { Mean Intensity of Transitive } \\
\text { Other Attributions divided by } 3\end{array}$ & $\begin{array}{l}+1.0 \text { optimistic to } \\
-1.0 \text { pessimistic }\end{array}$ \\
\hline $\begin{array}{l}\text { P-3. POLITICAL FUTURE (Predict- } \\
\text { ability of Others Tactics) }\end{array}$ & $\begin{array}{l}1 \text { minus Index of Qualitative } \\
\text { Variation** for Other Attributions }\end{array}$ & $\begin{array}{l}1.0 \text { predictable to } \\
0.0 \text { uncertain }\end{array}$ \\
\hline $\begin{array}{l}\text { P-4. HISTORICAL DEVELOPMENT } \\
\text { (Locus of Control) }\end{array}$ & $\begin{array}{l}\text { Self }(\mathrm{P} 4 \mathrm{a}) \text { or Other }(\mathrm{P} 4 \mathrm{~b}) \text { Attributions } \\
\square[\text { Self plus Other Attributions }]\end{array}$ & $\begin{array}{l}1.0 \text { high to } 0.0 \\
\text { low self control }\end{array}$ \\
\hline $\begin{array}{l}\text { P-5. ROLE OF CHANCE } \\
\text { (Absence of Control) }\end{array}$ & $\begin{array}{l}1 \text { minus [Political Future } \mathrm{x} \\
\text { Historical Development Index] }\end{array}$ & $\begin{array}{l}1.0 \text { high role } \\
\text { to } 0.0 \text { low role }\end{array}$ \\
\hline
\end{tabular}

political conflict, and the nature of other actors. In turn, VICS computes a simple ratio of the frequency of positive to negative utterances the political actor makes when talking about others in the political universe. This index varies from -1 to +1 with lower scores indicating that the political actor sees others as being more hostile in the political universe, whereas higher scores indicate that that the political actor sees others as being more friendly. The specific formula for this index is the percentage of positive utterances about others minus the percentage of negative utterances about others.

The second philosophical index looks at the prospects for realizing fundamental political values (P-2). This index goes beyond the balance of cooperative and conflictual utterances to include the way the political actor perceives the intensity of others actions. To calculate this index, VICS weighs each verb according to the intensity value of its coding category $(-3$ to +3$)$. Next, the result is divided by the total number of coded verbs. In turn, this index varies from -1 (pessimistic) to +1 (optimistic). The specific formula for this index is the mean intensity of utterances about others divided by three, which makes its values consistent with the other 
balance indices maximum value of +1 .

The third philosophical index is the predictability of the political universe (P-3). VICS assesses predictability by using a dispersion measure that calculates the variation in the distribution of observations across the scale of six verb categories $(-3$ to +3$)$ when the subject is talking about other actors; the dispersion measure used by VICS is the Index of Qualitative Variation (IQV; Watson and McGraw 1980, 88). ${ }^{3}$ The wider the variety of actions the subject attributes to others in the political universe, the less predictable are their actions. Similarly, if the political actor sees other engaging primarily in one or two categories of action, then the subject believes that others' actions are more consistent and thus more predictable. The specific formula for this index is one minus the IQV, which varies between 0 and 1 . Lower score indicate less predictability, whereas higher scores indicate perceptions of more predictability.

The fourth philosophical index is control of historical development (P-4). To measure this cognition, VICS computes a simple ratio of the number of self attributions to the number of actions that self attributes to others. This is because, as noted in the previous chapter, this is the only philosophical belief in an operational code that is concerned with what the subject believes about self and other. This index varies between 0 and 1. Lower scores indicate that the subject sees control residing more with others, which higher scores sees self as having more control. The specific formula for the P-4 index is the number of self utterances divided by the sum of self utterances plus other utterances.

The fifth and final philosophical index is the role of chance. As I explained in chapter three, this belief is a logical function of P-3 and P-4. In turn, VICS calculates this index by simply multiplying P-3 by P-4. The greater the product of this calculation, the lesser the role of

\footnotetext{
${ }^{3}$ The IQV is a ratio of the number of different pairs of observations in a distribution to the maximum number of possible number of different pairs for a distribution with the same number of cases $(\mathrm{N})$ and the same number of variable classification (Watson and McGraw 1980, 88).
} 
chance. VICS transforms this interaction term to make the direction of the index vary so that higher scores indicate a higher role assigned to chance and lower scores indicate a lower role. The role of chance index ranges from 0 to 1 . The specific formula for P-5 is one minus the product of the Predictability Index (P-3) times the control index (P-4).

\section{The Coding Approach: Hand Coding or Computer Coding?}

Initial work using VICS was coded by hand (Crichlow 1998; Schafer and Walker 2001; Schafer, Walker, and Young 2002; Walker, Schafer, and Young 1999). Hand coding begins after the researcher gathers appropriate texts relevant to the research question at hand. The first step is to identify the subject under investigation, audience, occasion for the speech act, and other situational variables that might be of interest to the research question. Next, the researcher highlights or underlines the verbs in the text and then must apply the appropriate code to each verb. The researcher must clearly identify the transitive verb or verb-based phrase, specify the positive or negative valence of each verb, specify whether the verb is word or deed, and specify the appropriate final coding category for the verb from the six possibilities: Punish (-3), Threaten $(-2)$, Oppose (-1), Support $(+1)$, Promise $(+2)$, and Reward (+3). The final step is to identify the subject as self or other and add other contextual information as appropriate to the research question. Schafer and Walker $(2006,39)$ note that it is imperative that researchers follow these steps with as much care as possible, which literally means moving through the text one verbbased utterance at a time. Researchers must also specify a data line for each verb.

The major advantage of hand coding is that it can subjectively interpret obscure phrases. However, like early operational code methodologies, hand coding also suffers from several shortcomings. It is labor intensive, time consuming, and subject to human error. Human error, which can act as a confounding factor in statistical analyses, can result from fatigue, personal 
political biases, learning effects, and other idiosyncratic stylistic differences (Schafer and Walker 2006a, 38). Because of these shortcomings, the reliability of VICS data coded by hand is brought into question. It is very rare, if not nearly impossible, to have perfect agreement among several different coders.

To help overcome the disadvantages of hand coding, Social Science Automation (http://www.socialscience.net/) has developed an automated, full-language parser software program known as Profiler Plus. Profiler Plus works to uncover critical information with a speed and accuracy impossible using manual techniques. The main objective of this software is to facilitate the at-a-distance, psychological assessment of political actors. Nearly all recent analyses in this field have used Profiler Plus (e.g., Drury 2006; Feng 2006; Malici 2005, 2006; Malici and Malici 2005a; Marfleet and Miller 2005; Robison 2006; Thies 2006). The program continues to be updated and the company has, with the assistance of primary researchers in the operational code field, developed large dictionaries of verbs, verb forms, as well as coding and parsing rules for VICS.

Detailed instructions for automated coding with Profiler Plus as a general software program are available from Social Science Automation. As for procedures specific to VICS, the process begins with collecting texts that are appropriate for the research question at hand. Given that we are dealing with automated coding via computer, texts must be in digital format. Texts that are not already in digital format must be manually typed into word processors, or the researcher has to scan and edit the originals and convert them to digital format. Next, Profiler Plus' document markup procedure is used to mark-up the text and embed certain contextual information. Mark-up is necessary so that the program ignores things such as endnotes, footnotes, and any other material that the researcher does not want coded but does want 
preserved to maintain context. Other contextual information can include the name of the speaker, the date of the speech act, and the type of speech act. Finally, after the researcher activates the automated coding function, Profiler Plus differentiates "self" and "other" automatically by relying on self-preferential pronouns (I, me, we, us, ect.) in each verb-based utterance. If a self-referential pronoun is not identified, Profiler Plus concludes that the subject is referring to others. This is done because self-referential pronouns account for a large number of utterances that political actors make regarding "self" (Schafer and Walker 2006a, 42).

Automated coding has several advantages. The most obvious ones are that it is fast, efficient, and labor friendly. Computer-based coding also essentially eliminates human biases and error. Granted, the computer cannot code everything with absolute accuracy. If the computer does make an error, this will repeated throughout the automated coding procedure. However, this also means that errors should not generally bias the data. That is, even though computer coding can be wrong, it is always going to be wrong in the same direction/ways. This is an obvious advantage over human coding. In the end, the major advantage of computer-based coding is that it provides 100 percent reliability, whereas human coding generally does not.

Given that automated coding provides numerous advantages, it is not surprising that hand coding has fallen out of favor and is only rarely used. The dominance of computer coding has also been facilitated by the fact that although the underlying logic of hand coding and automated coding is the same, the two approaches rely on quite different coding rules. Each approach is likely to produce significantly different results across texts (Schafer and Walker 2006, 39). Therefore, it is inappropriate to compare data coded by hand to that coded by a computer (Ibid., 39). It is also inappropriate to compare these indices to those generated via processes utilizing

\footnotetext{
${ }^{4}$ Many researchers will manually go through their sample of speech acts and note the non-pronouns that the subject uses with frequency to refer to "self" and "self"s" in-groups. Subject specific "self" dictionaries are then developed to tell Profiler Plus to code utterance as "self" when it sees one of those references as the subject of the verb.
} 
programs other than Profiler Plus or processes that do not employ custom self dictionaries

(Picucci 2008, 153). In conclusion, because I am only able to select one coding approach, this dissertation employs computer based coding rather than hand coding to generate VICS data on the worldview of U.S. intelligence. ${ }^{5}$

\section{Data Source}

Which data source should I extract speech acts from? ${ }^{6}$ Providing an answer to this question is complicated by the fact that the amount of publicly available, primary source documentation for scholarly research on intelligence issues is limited. The cloud of secrecy that the IC operates under is the primary cause of this. ${ }^{7}$ Furthermore, the IC characterizes itself as a "federation" of its member elements. This means that its overall structure is better characterized as a confederation due to its lack of a well-defined, unified leadership and governance structure (Turner 2004, 51). ${ }^{8}$ The IC's confederal structure makes it difficult for me to find a source that can accurately aggregate the core beliefs that the various IC agencies collectively hold about world politics given that it can lead to construct validity problems. ${ }^{9}$

Nevertheless, one potential data source is the formal, prepared statements of the Director of Central Intelligence. ${ }^{10}$ For over fifty years, the DCI coordinated the activities of all agencies

\footnotetext{
${ }^{5}$ The coding scheme that I will use is provided free of charge by Social Science Automation on their website. I will also incorporate a "self dictionary" to ensure that Profiler Plus codes each verb-based utterance as accurately as possible. The dictionary will include the full names and acronyms of all IC agencies as well as the following: "all agencies," "all IC agencies," "American intelligence," "intelligence community," "intelligence agencies," "most agencies," "several agencies," "this assessment," "this estimate," and "U.S.intelligence."

${ }^{6}$ Speech acts refer to utterances within documents (data sources) that serve as functions in communication. Put simply, a speech act is an act that a speaker performs when making an utterance. In this dissertation, a speech act refers to verb-based utterances (the act) within its data source.

${ }^{7}$ Granted, U.S. intelligence is the most transparent intelligence service in the world. Indeed, the U.S. is one of the few countries in the world that allows and sometimes encourages the public to discuss and debate American intelligence activities (Turner 2005, 229). However, this perspective is a recent one dating only to the 1980s. Moreover, the U.S. still maintains a great deal of secrecy over a substantial number of intelligence operations to protect sources and methods (Ibid., 229).

${ }^{8}$ This applies to the pre-DNI world, as well as the DNI era.

${ }^{9}$ Construct validity seeks agreement between a theoretical concept and a specific measuring device or procedure.

${ }^{10}$ The creation of the position of Director of National Intelligence (DNI) in April 2005 ended the 57-year reign of
} 
within the IC and took overall responsibility for gathering information from other intelligence agencies, analyzing the separate pieces of information from each source, and providing intelligence estimates to the president and the president's advisers. At the same time, the DCI served as the senior intelligence official, head of the intelligence community, and principle intelligence advisor to the president and his advisors (Lowenthal 2006, 30). This made the DCI the chief spokesperson for the IC as a whole. This suggests that by focusing on the formal, prepared statements of the DCI, I might be able to aggregate the worldview of the IC with reasonable accuracy.

There are several problems with using DCI speeches as my data source, however. For one, the DCI had much less authority to run the IC than any other intelligence chief had to run his own agency (Johnson and Wirtz, eds. 2004, 27). The DCI was able to manage the IC on paper, but, in practice, the DCI only had real authority over his own agency (the CIA). This hurts the ability of DCI speeches to truly represent the views of the entire IC. A second issue of concern is that these individuals are attempting to be deceptive (Tetlock and Manstead 1985). Indeed, "impression management" is a major tactic used by intelligence agencies to deceive enemies of the state. A third issue of concern is that although several DCI speeches are readily available through various sources, finding speeches prior to the mid-1990s is extremely difficult. ${ }^{11}$ This prevents me from testing my hypothesis that the worldview of U.S. intelligence is specific and longstanding. Finally, only seven out of seventeen DCIs made a concerted effort to include the interests and preferences of the various intelligence agencies into their policy

the DCI as the nation's chief intelligence officer. Although the DNI and DCI job functions are similar, there are subtle differences. For example, the DNI has greater authority of budgetary and personnel decisions. However, DNI speeches cannot be the sole data source since the position has only existed for just over two years. Moreover, it cannot be used in conjunction with DCI speeches since it would create multiple group threats to internal validity.

${ }^{11}$ The one exception is speeches given by Bill Casey, who served as DCI from 1981 to 1987 . The book Scouting the Future: The Public Speeches of William J. Casey (Casey 1989) provides researchers with several public speeches given by Casey as DCI. 
decisions (Garthoff 2005). This includes Richard Helms, James Schlesinger, William Colby, George H.W. Bush, Stansfield Turner, George Tenet, and Portor Goss (Ibid.). When taken together, these issues of concern indicate that using the formal, prepared statements of DCIs as my data source would be detrimental to statistical analysis and lead to numerous reliability and validity problems.

Fortunately, there is one other type of data source that is publicly available and that VICS can score attribution patterns from. The data source that I am referring to is declassified National Intelligence Estimates (NIEs). NIEs are the IC's most authoritative written judgments concerning national security issues. They contain the coordinated judgments of the IC regarding the current state of play, as well as the likely course of future events. ${ }^{12}$ NIEs are reviewed and approved for dissemination by the National Intelligence Board (NIB), which is comprised by the Director of National Intelligence and other senior leaders within the IC. Once approved by the NIB, NIEs are presented to the President and senior policymakers. For an overview of the NIE writing process see Bruno and Otterman (2008).

NIEs cover an array of international issues. This ranges from military to technological to economic to political trends. NIEs were first produced in 1950 by the Office of National Estimates (ONE), although the concept of an "estimative" intelligence report was established by the National Security Act of 1947 following the surprise invasion of South Korea by North Korean troops. In 1973, the ONE was superseded by National Intelligence Officers to help increase interagency collaboration. This group of substantive experts eventually became the National Intelligence Council (NIC) in 1979. Today, the NIC continues to produce NIEs and is the center for mid-term and long-term strategic thinking within the IC.

\footnotetext{
${ }^{12}$ This is what is known as estimative intelligence, which is a category of intelligence analysis that attempts to project probable future developments and their implications for U.S. interests.
} 
Declassified NIEs are an excellent choice for this dissertation's data source for numerous reasons. Most obviously, declassified NIEs are publically available and can reflect and aggregate with reasonable accuracy the core beliefs that the various IC agencies hold about world politics. This is not to say that it can produce a perfect aggregation. Certain agencies, such as the Central Intelligence Agency, have more input into NIEs than others, such as Coast Guard Intelligence. Furthermore, several agencies are likely to have strongly held and diametrically opposed views on key issues within an estimate (Lowenthal 2006, 122). Still, declassified NIEs are the only publically available data source that can minimize construct validity problems. This is because all IC agencies that focus on foreign intelligence are involved in the process that leads to the final NIE product. Everyone involved in the NIE also generally finds some way to agree when disagreements over key issues occur. As Lowenthal notes, "as a rule, policymakers prefer consensus views, which save them from having to go through numerous shades of opinion on their own...Thus, there has always been some impetus to arrive at a consensus if possible" (123).

It is important to point out that the need to arrive at consensus could lead to groupthink (Ibid., 23). For example, regarding the IC's analysis of Iraqi WMD, the Senate Select Committee on Intelligence held that IC analysts did not reexamine their assumptions rigorously enough and thus lapsed too easily into agreement. Unfortunately, the threat of groupthink is likely to be ever-present in the creation of NIEs, as well as any coordinated IC product, due to political pressures. There are several ways that NIEs can be affected by politics. A potential negative reaction from policymakers could cause IC agencies to censor or hold back their opinions during the production process. Or, an influential individual involved in the NIE writing process could have career interests that could be realized more easily if they advocated an 
opinion or outcome preferred by policymakers or a more senior intelligence official. Despite the threat of groupthink, I still believe that declassified NIEs are an excellent choice for this dissertation's data source. When compared to other potential data sources, declassified NIEs are the only data source that can minimize construct validity problems.

Declassified NIEs are also advantageous because they contain several speech acts. In particular, they contain numerous verbal expressions of probability (Kent 1964). Moreover, NIEs, especially the key judgments section within them, contain a large number of selfpreferential pronouns (I, me, we, us, ect.) in their verbal expressions about current and future international events. This observation has led to several studies on the language of NIEs (e.g., Ford 1993; Kent 1964; Kesselman 2008; Russell 2004; Wark 1964). The most recent study by Rachel Kesselman analyzes trends in verbal probability expressions in NIEs by conducting a comprehensive statistical analysis from 1950 through 2007. The large of speech acts, along with the fact that there are hundreds of NIEs readily available online for scholarly research, means that using declassified NIEs as my data source will allow me to test my continuity hypothesis.

In the end, the advantages that declassified NIEs present outweigh their disadvantages. Therefore, declassified NIEs, specifically the key judgments section within them, will serve as this dissertation's data source. Although they are by no means a perfect data source, they can be used aggregate the core the beliefs that the various IC agencies hold about world politics with reasonable accuracy. This is in contrast to the formal, prepared statements of DCIs, whose use would have led a variety of validity and reliability problems. I would have liked to select a source that would eliminate the problem of construct validity, but this is unavoidable since political pressures are likely to be ever-present in the creation of any coordinated IC product. 


\section{Sampling Frame}

To generate a sampling frame of NIEs, I first gathered as many NIEs that I could find from various online sources. ${ }^{13}$ The time period here was 1950 through 2007 . Next, I eliminated any NIE from this list that did not meet the following selection criteria: (1) the subject and object were international in scope; (2) the focus of interaction was a political issue; (3) the words and deeds were cooperative and conflictual; (4) the key judgments section contained at least 1500 words. ${ }^{14}$ The first three criterions are standard for operational code analyses that focus on world politics (Walker, Schafer, and Young 1998, 182). As for the fourth criterion, this is the minimum number of words necessary for VICS to provide enough data per speech act to construct the operational code indexes (Walker, Schafer, and Young 1999, 614). After eliminating the NIEs that did not meet the selection criteria, as well as Special National Intelligence Estimates (SNIEs), ${ }^{15}$ I was left with 59 declassified NIEs (see Appendix A). This represented my testable population.

I would have liked to use random sampling to create my sampling frame. ${ }^{16}$ However, this was not possible given that an analysis of variance test (ANOVA), which is one of the statistical procedures that I will employ to analyze the VICS data, requires equal sample sizes from two or more categories of data. I had to use stratified random sampling instead. Stratified

\footnotetext{
${ }^{13}$ Online sources included the CIA's Freedom of Information Act electronic reading room (http://www.foia.cia.gov/), the DNI's website (http://www.dni.gov/), the Federation of American Scientists website (http://www.fas.org/), GlobalSecurity.org (http://www.globalsecurity.org), and the NIC's website (http://www.dni.gov/nic/NIC_home.html).

${ }^{14}$ This is minimum number of words necessary for VICS to provide enough data per speech act to construct the operational code indexes (Walker, Schafer, and Young 1999, 614)

${ }^{15}$ SNIEs are NIEs scheduled ad hoc and on short notice, usually to address a more narrowly defined issue. Although SNIEs are very similar to regular NIEs in terms of their language and purpose, I have decided to exclude SNIEs because they are likely to suffer from strong in-group pressures toward conformity and cohesiveness given that they have to be produced very quickly in a stressful environment (Janis 1982). Furthermore, since SNIEs focus on more narrowly defined issues, the key judgments contained in SNIEs are for the most part only attributable to the most relevant intelligence agencies involved in the process.

${ }^{16}$ Random sampling depends on selecting a random a sufficient sample of the population such that there is a high probability of reproducing the essential characteristics of the total population.
} 
random sampling involves dividing the population into homogeneous subgroups and then taking a simple random sample from each subgroup. The major advantage of this approach to sampling is that it assures that the analyst will be able to represent not only the overall population, but also key subgroups of the population (Hoover and Donovan 2001, 97). Stratified random sampling also has a high level of statistical precision (Ibid., 97).

I divided my population into six subgroups based upon five controlling factors to begin the process of stratified random sampling. ${ }^{17}$ The control factors were as follows: (1) the signing of the Strategic Arms Limitation Treaty (SALT I) in May 1971, which marked the official beginning of détente; ${ }^{18}$ (2) the Soviet invasion of Afghanistan in December 1979, which marked the official end of détente; (3) Mikhail Gorbachev's challenge to the practice of Cold War international politics, which began in March $1985 ;{ }^{19}$ (4) the end of the Cold War in December 1991; (5) and 9/11. These control factors represent significant international events that would have likely "shocked" the IC's core belief system about world politics. That is, each event would have caused U.S. intelligence to ponder whether or not it needs to alter its worldview so that it can produce intelligence that is relevant in the changing international environment.

After dividing my population of NIEs into the six subgroups, I had to generate a random

\footnotetext{
${ }^{17}$ The six control groups are December 1950-April 1971, May 1971-November 1979, December 1979-February 1985, March 1985-December 1991, January 1992-September 11, 2001, and September 12, 2001-December 2007.

${ }^{18}$ Détente is a French term, meaning a relaxing or easing. During the Cold War, détente was ushered in to reduce hostilities between the U.S., Soviet Union, and China. For instance, in February 1972, U.S> President Richard Nixon traveled to Beijing, China and met with Mao Zedong and Chou En-Lai. Nixon and his Secretary of State Henry Kissinger then announced a stunning rapprochement with Mao's China. Moreover, several international agreements were reached over the next few years, such as the Helsinki Accords and second Strategic Arms Limitation Treaty (SALT II). Therefore, détente ushered in an era of cooperation during the Cold War that was unthinkable during the 1950s and most of the 1960s.

${ }^{19}$ During his tenure the Soviet Union stopped playing "power politics," despite the fact that it was the Kremlin's foreign policy strategy of choice since the Cold War's inception. ${ }^{19}$ In particular, Gorbachev convinced U.S. President Ronald Reagan that his past perception of the Soviet threat was no longer warranted (Malici 2006). This was achieved through a foreign policy of improving relations and trade with the West by reducing Cold War tensions via altercasting, as well as through the reform programs Perestroika and Glasnost. ${ }^{19}$ Ultimately, Gorbachev transformed Cold War international relations by making the relationship between the U.S. and the Soviet Union more cooperative than conflictual.
} 
sample in each subgroup. However, I was only able to do this for the first subgroup (December 1950-April 1971). This was because the remaining subgroups had relatively short time periods and contained a small number of NIEs. These made random sampling unnecessary, which meant that I only randomly selected NIEs from the first subgroup. This left me with a sampling frame that had five declassified NIEs in each subgroup for a total sample size of 30 (see Appendix B). At this point, I was satisfied with the overall size of my sample. As Schafer and Walker (2006) explain, "it is not necessary to code every speech in a subject's career to construct a good profile" (44). Instead, as little as 10 speeches are sufficient to create a basic profile (Ibid., 44). Nevertheless, even though I was satisfied with my sample size, this is not to say that this outcome was ideal. Rather, I felt it was unavoidable given that there is no publically available data source that I could have used to inflate my population without comprising the construct validity of my sampling frame. ${ }^{20}$

\section{Analyzing the VICS Data}

I will extrapolate the IC's philosophical beliefs about world politics from the VICS data by generating the mean of the subgroup scores within my sampling frame. I will sum the subgroup scores and divide the total by 6 . This will produce the average score for each of the five philosophical indices. These scores will either confirm or deny the five hypotheses presented in the previous chapter. Data will be interpreted based generally upon the balance indices presented in Figure 4.3. These scales illustrate the range of values associated with each philosophical index and the remaining descriptors used to interpret and anchor the scores. A particular score is anchored to an interpretation based on the distance between the score and the nearest descriptor. For example, a score of +.21 is anchored to the descriptor "Somewhat Friendly" on the nature of the political universe scale for P-1. This is because it is closest to the

\footnotetext{
${ }^{20}$ SNIEs could have been used, but were too small in number to make any real difference.
} 
+.25 on the continuum of possible balance descriptors. In turn, this score would be interpreted as follows: "The political actor has a somewhat friendly image of the political universe and the fundamental nature of their political opponents."

To test my first hypothesis, which states that the worldview of U.S. intelligence is specific and longstanding, I will run a one-way ANOVA at the .05 level of significance with time period (each subgroup) as a categorical factor. The one-way ANOVA test is a powerful and common statistical procedure in the social sciences. It is also one of the most popular forms of statistical analysis used by contemporary operational code scholars. A one-way ANOVA tests for differences among two or more independent groups. ${ }^{21}$ It is generally used when the researcher is comparing more than two samples or when the independent variable has more than two attributes. ANOVA identifies differences between groups by pooling the variance between the groups, which generates an F statistic. An F statistic is the ratio of the variance among the groups to the variance within the samples. A statistically significant F score indicates that there is significant variance between the groups, whereas a statistically insignificant F score indicates no significant variance between the groups. The statistical significance of the $\mathrm{F}$ score is indentified by the p-value. In this dissertation, a p-value greater than 0.05 will indicate a statistically significant $\mathrm{F}$ score. A p-value less than 0.05 will represent a statistically insignificant F score.

A one-way ANOVA makes three assumptions. First, it assumes that the observations within each group or category are independent (independence). Second, it assumes the population distributions are normally distributed (normality). And third, it assumes the population distributions have the same variances (homogeneity). If any of these assumptions are

\footnotetext{
${ }^{21}$ Typically, however, a one-way ANOVA is used to test for differences among at least three groups. This is since the two-group case can be covered by a T-test (Gossett 1908).
} 
violated, the validity and robustness of the results are brought into question. To ensure that my data meet ANOVA's assumptions, I will conduct a priori tests. Specifically, I will conduct a Shapiro-Wilk test to check the normality assumption and a Levine's test to check the homogeneity assumption. I do not need to run any statistical tests to check the independence assumption since the data in each subgroup were generated from separate samples of speech acts. I will run the appropriate statistical tests that correct for the violations in place of the standard one-way ANOVA if I find that the data violates one or both assumptions. These tests will discussed in the next chapter (data analysis), if necessary.

\begin{tabular}{|c|c|c|c|c|c|c|c|}
\hline \multicolumn{8}{|c|}{ FIGURE 4.3: BALANCE INDICES FOR THE FIVE PHILOSPHICAL BELIEFS } \\
\hline \multicolumn{8}{|c|}{ P-1: The Nature of the Political Universe (Hostile/Friendly) } \\
\hline HOSTILE & & & & & & & FRIENDLY \\
\hline Extremely & Very $\mathrm{I}$ & Definitely & Somewhat & Mixed & Somewhat & Definitely Very & Extremely \\
\hline-1.0 & -.75 & -.50 & -.25 & 0.0 & +.25 & $+.50 \quad+.75$ & +1.0 \\
\hline \multicolumn{8}{|c|}{ P-2: Realization of Political Values (Pessimism/Optimism) } \\
\hline PESSIMISM & & & & & & & OPTIMISM \\
\hline Extremely & Very I & Definitely & Somewhat & Mixed & Somewhat & Definitely Very & Extremely \\
\hline-1.0 & -.75 & -.50 & -.25 & 0.0 & +.25 & $+.50 \quad+.75$ & +1.0 \\
\hline \multicolumn{8}{|c|}{ P-3: Predictability of Political Future (Very Low/Very High) } \\
\hline PREDICTABILITY & & & & & & & PREDICTABILITY \\
\hline Very Low & & Low & & Medium & & High & Very High \\
\hline 0.0 & & .25 & & .50 & & .75 & 1.0 \\
\hline \multicolumn{8}{|c|}{ P-4: Control Over Historical Development (Very Low/Very High) } \\
\hline CONTROL & & & & & & & CONTROL \\
\hline Very Low & & Low & & Medium & & High & Very High \\
\hline 0.0 & & .25 & & .50 & & .75 & 1.0 \\
\hline \multicolumn{8}{|c|}{ P-5: Role of Chance (Very Low/Very High) } \\
\hline CHANCE & & & & & & & CHANCE \\
\hline Very Low & & Low & & Medium & & High & Very High \\
\hline 0.0 & & .25 & & .50 & & .75 & 1.0 \\
\hline
\end{tabular}

Once I have carried out the ANOVA test(s) through a computerized statistical software package, I will report my data and determine what it has to say about my hypothesis that the 
philosophical beliefs that U.S. intelligence holds about world politics are specific and longstanding. If I find that there are significant differences in any of the index data between the subgroups, I will run post-hoc analyses to determine where the specific differences occurred. ANOVA only determines if there are differences between groups, not what the exact differences are. In other words, ANOVA does not indicate which specific group pair(s) are the ones where statistical differences occur. As with the corrective tests for assumption violations, post-hoc analyses will be discussed in the next chapter if they are deemed necessary.

\section{Chapter Summary}

This chapter identified the methodology and data that this dissertation uses to test its hypotheses. It began by briefly discussing the history of operational code methodology, which demonstrated that the Verbs in Context System of content analysis is the most reliable and robust method available to quantify the core beliefs that the IC holds about world politics. Next, I presented the logic behind VICS and explained how operational code indices are calculated. I then compared and contrasted the two coding approaches available and determined that this dissertation will utilize computer coding to generate VICS data on the worldview of U.S. intelligence. In the sections that followed, I identified my data source (declassified National Intelligence Estimates) and sampling frame. Finally, the statistical methods that this dissertation will use to analyze the VICS data and check its hypotheses were identified and discussed. The next chapter presents the analysis of my data. 


\section{CHAPTER FIVE}

\section{DATA ANALYSIS}

This chapter presents and discusses the statistical data that I obtained after implementing the research design outlined in the previous chapter. In effect, this chapter assesses the accuracy of my theory and hypotheses. To review, the theory of this dissertation is that the core beliefs that the IC holds about world politics (the worldview of U.S. intelligence) underlie several U.S. intelligence norms. I derive six hypotheses from this theory. Hypothesis one $(\mathrm{H}-1)$ posits that U.S. intelligence has a hostile image of the political universe and the fundamental nature of their political opponents. Hypothesis two (H-2) states that U.S. intelligence has an optimistic view regarding the realization of its fundamental political values. Hypothesis three (H-3) declares that U.S. intelligence believes that the political future is predictable. Hypothesis four (H-4) argues that U.S. intelligence believes that it has a low degree of control over historical development. Hypothesis five (H-5) posits that U.S. intelligence does not attribute a great role to chance. Finally, my sixth proposition (H-6) states that the worldview of U.S. intelligence is specific and longstanding.

This chapter is broken up into three sections. First, I present VICS data on the IC's philosophical beliefs about world politics, which I use to gauge the accuracy of hypotheses one through five. These data were extrapolated from verb-based utterances within thirty declassified National Intelligence Estimates. As I demonstrated in the previous chapter, NIEs are the only publically available source that can reflect and aggregate the beliefs that all IC agencies hold about world politics with reasonable accuracy. The second section of this chapter presents the results of several one-way ANOVA tests, which are used to assess my hypothesis that the IC's worldview is specific and longstanding. Next, I discuss the exactness of my theory and 
hypotheses in light of my findings. I conclude with a chapter summary.

\section{VICS Data on the Philosophical Beliefs in an Operational Code}

The average word count for the thirty speech acts was 1835 and the average number of verbs coded per NIE was 58. Overall, references to the "self" were small in number relative to the number of references coded as "other." There were 1562 verbs coded as "other" and 170 verbs coded as "self." Nevertheless, this only affected the scores of the P-4 and P-5 indices. This is because these are the only two philosophical indices that rely upon the ratio of the number of "self" and "other" references. Moreover, the NIEs were generally more explicit in their references to "self" than in their references to "other." "Self" references in each verb-based utterance were generally pronouns such as "us" and "we," whereas pronouns such as "their" or "it" were scarce. It is therefore unlikely that there was systematic undercounting or overcounting of "self" references. The extensive "self" dictionary that I developed and embedded into the coding scheme buttresses this contention.

On the other hand, it is probable that there was a systematic overcounting of "other" references since these were often implicit in each verb-based utterance. Rather than using pronouns such as "their" or "it" when speaking about "others" in the political universe, the NIEs used phrases like "the group" and "the coalition." Because Profiler Plus automatically concludes that the speaker is referring to others when it does not identify a self-referential pronoun in an utterance, it likely overcounted the actual number of references to the "other" in the NIEs. However, since overcounting of "other" references would be uniform for each NIE coded due to the employment of computer coding, this would only affect the P-4 and P-5 index scores. In the end, although completely accurate identification of "self" and "other" pronouns would have been optimal, the pitfalls of automated coding still outweigh the pitfalls of hand 
coding.

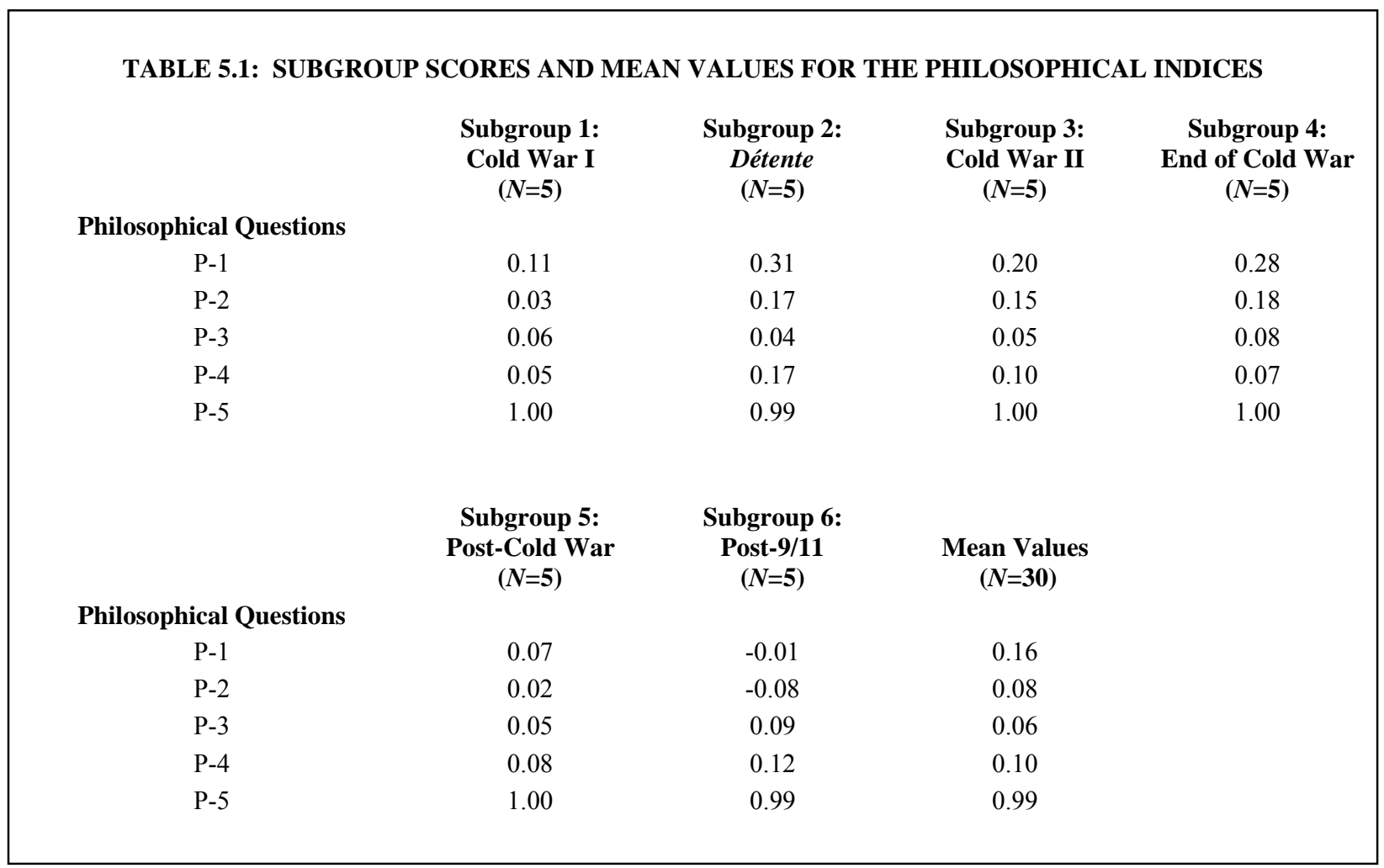

Table 5.1 summarizes the values for each subgroup and the mean values of the subgroup scores. The mean values demonstrate that the IC has a mixed view about the nature of the political universe (friendly/hostile) $(\mathrm{P}-1=0.16)$ and about the realization of their political values (optimistic/pessimistic) $(\mathrm{P}-2=0.08)$. The scores also show that the U.S. intelligence believes that the chance of accurately predicting the political future is very low $(\mathrm{P}-3=0.06)$, that it has very little control over historical development $(\mathrm{P}-4=0.10)$, and it attributes a very high role to chance (P-5=0.99). These results are generally consistent across the six subgroups and more or less mirror the values of the subgroup scores. The only deviations occur in subgroups two, four, and six in regards to the P-1 index. In subgroups two and four, the data states a somewhat friendly view of the political universe $(\mathrm{P}-1=0.31$ and $\mathrm{P}-1=0.28$, respectively). In subgroup six, the $\mathrm{P}-1$ score (-0.01) still indicates a mixed view about the nature of the political universe. However, 
this score also indicates that political opponents are slightly more hostile than friendly.

\section{ANOVA Results}

Prior to conducting a one-way ANOVA test to determine if there were statistically significant differences in the philosophical index scores between the six subgroups, I first checked ANOVA's normality and homogeneity assumptions. To ensure that the sample within each subgroup was normally distributed, I conducted a Shapiro-Wilk test with an alpha level of 0.05. The null hypothesis for this test was that the data were normally distributed. A Levene's test was used to assess the equality of variance in the different index populations with an alpha level of 0.05 . The null hypothesis for this test was that the population variances were equally distributed. Table 5.2 presents the results of these a priori analyses. The p-values of the Shapiro-Wilk test demonstrate that the samples within P-3, P-4, and P-5 $(\mathrm{p}=0.001, \mathrm{p}=0.000$, and $\mathrm{p}=0.000$, respectively) were not normally distributed. Therefore, the data within these groups violate the normality assumption. The results of the Levene test indicate that the variances in each of the index populations were unequal. The P-2 variance score was 0.001 , the P-4 variance score was 0.002 , and the P-5 variance score was 0.000 . This means that the homogeneity assumption was also violated.

Due to the preceding, I was unable to run a standard one-way ANOVA test to check my hypothesis that the philosophical beliefs that the IC holds about world politics are specific and longstanding. I ran the risk of drawing false conclusions from my analysis. That is, I might falsely conclude that there is a difference(s) between groups when their actually is none (a type 1 error). ${ }^{22}$ To avoid committing a type 1 error, I ran a Kruskal-Wallis ANOVA test and a Welch

\footnotetext{
${ }^{22}$ Some statisticians argue that if you are conducting a between subjects ANOVA, and you have equal or nearly equal numbers of participants contributing to each mean, then you can risk quite extreme violations of normality and homogeneity without substantially increasing your chances of drawing false conclusions. Specifically, if the skew for each mean in your data is in the same direction, and the biggest variance is no more than four times the smallest
} 
ANOVA test instead. I used an alpha level of 0.05 for each of these tests. The former is a nonparametric analogue to ANOVA used to correct for violations of the normality assumption. A Kruskal-Wallis ANOVA mirrors a standard one-way ANOVA except for the fact that it does not make any assumptions about how the data are distributed. The latter is intended for use with two or more samples that have unequal variances. It tests for differences in the means of the groups, while allowing for unequal variances across groups. When taken together, a KruskalWallis ANOVA and a Welch ANOVA represent a useful alternative to a one-way ANOVA. This is because they make almost the least amount of assumptions about the data under investigation (Miller and Whicker 1998, 233-235).

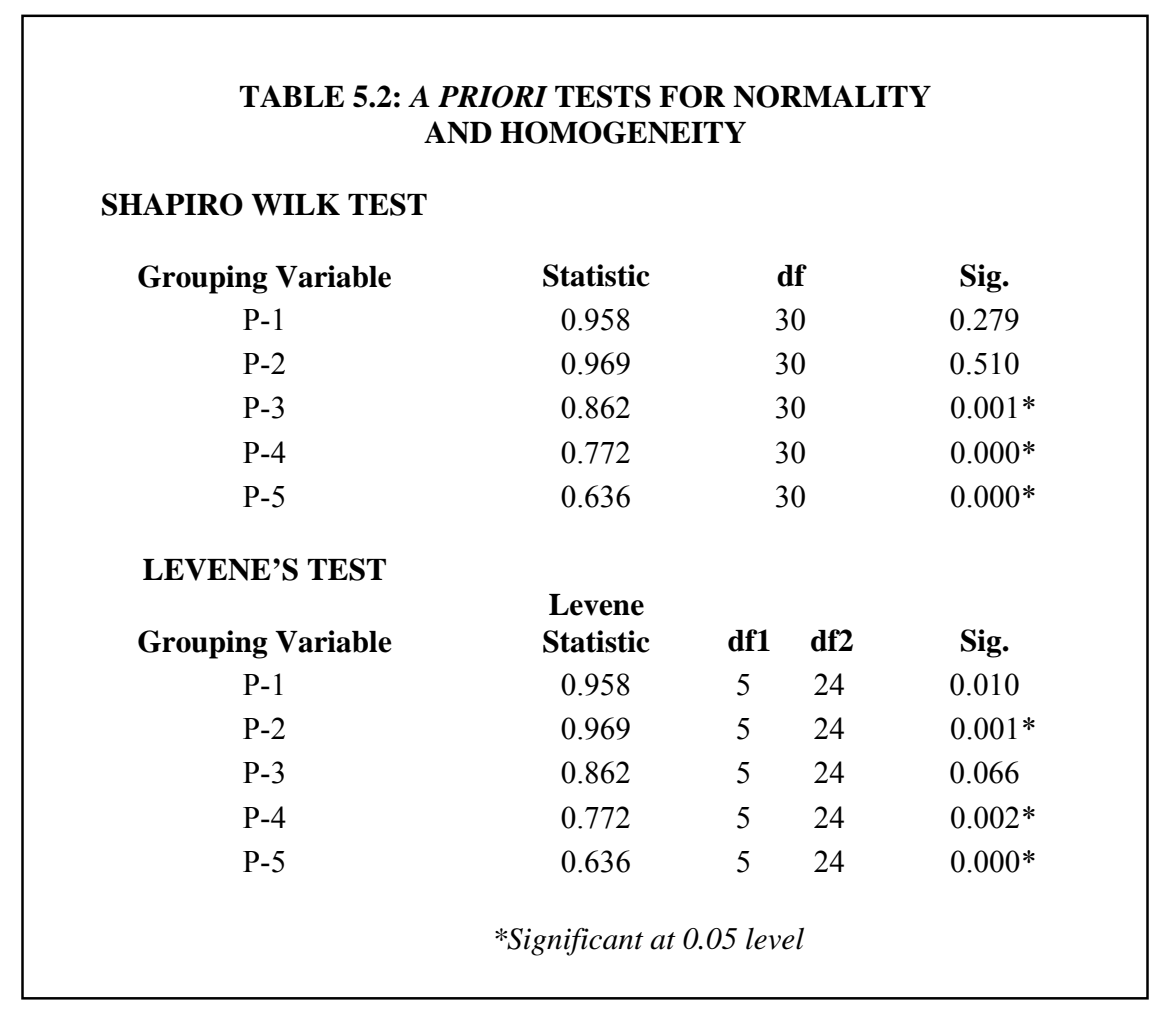

The results of the Kruskal-Wallis and Welch ANOVAs are presented in Table 5.4. Both tests found that there were no significant differences in the philosophical index scores between

variance, then running a one-way ANOVA despite assumption violations is fine. Nevertheless, my data do not meet these criteria. This means that it would be inappropriate for me to run a standard one-way ANOVA. 
the six subgroups. The null hypotheses for both tests were not rejected. For the Kruskal-Wallis ANOVA, all p-value scores are greater than the significance level of 0.05 . The p-value for P-1 was 0.280 , the $\mathrm{p}$-value for P-2 was 0.151 , the $\mathrm{p}$-value for P-3 was 0.154 , the $\mathrm{p}$-value to P-4 was 0.349, and the p-value was 0.081. Similarly, the p-value scores for the Welch ANOVA were also greater than 0.05 . The p-value for P-1 was 0.212 , the p-value for P-2 was 0.096 , the p-value for P-3 was 0.186 , the p-value to P-4 was 0.243 , and the p-value for P-5 was 0.069 . Given that there are no statistically significant differences in the philosophical index scores between the six subgroups, no post-hoc analyses were necessary since differences that needed pinpointing did not exist and no unexpected effects were found.

\begin{tabular}{|cccc|}
\hline \multicolumn{2}{|c}{ TABLE 5.3: ANOVA RESULTS } & \\
KRUSKAL WALLIS ANOVA & & & \\
Grouping Variable & Chi-Square & df & Sig. \\
P-1 & 6.279 & 5 & 0.280 \\
P-2 & 8.095 & 5 & 0.151 \\
P-3 & 8.042 & 5 & 0.154 \\
P-4 & 5.581 & 5 & 0.349 \\
P-5 & 9.795 & 5 & 0.081 \\
WELCH ANOVA & & & \\
Grouping Variable & & & \\
P-1 & Statistic & df2 & Sig. \\
P-2 & 1.724 & 10.691 & 0.212 \\
P-3 & 2.530 & 10.456 & 0.096 \\
P-4 & 1.838 & 11.038 & 0.186 \\
P-5 & 1.587 & 10.903 & 0.243 \\
& 2.845 & 10.972 & 0.069 \\
\hline
\end{tabular}

\section{Discussion}

The above analyses provide mixed support for this dissertation's theory and hypotheses. On the one hand, the results of the Kruskal-Wallis and Welch ANOVAs provide strong support for my hypothesis that the worldview of U.S. intelligence is specific and longstanding. Indeed, the p-scores from both analyses leave little to no doubt that the philosophical indices generated 
from VICS coding of verb-based utterances within thirty National Intelligence Estimates are temporally stable. Therefore, one important finding of the data analysis is that the IC's core beliefs about world politics are more than likely not prone to fluctuation over time in responses to changes in context. Although this may not be for the exact reasons laid out in chapter three, it is probably this dissertation's most valid finding. This is because, regardless of the document coded, the actors involved in the writing process change over time. Moreover, world politics is unstable given that new events constantly occur and new types of political actors are constantly emerging. Since understanding world politics is at the heart of the IC's mission, documents that reflect the IC's core cognitions about foreign affairs and global issues among state and non-state actors in all probability take into account events that could potentially "shock" its worldview.

The data also provide support for my hypothesis that U.S. intelligence believes that it has a low degree of control over historical development. An index score of 0.10 is anchored to the descriptor "Very Low" on the control over historical development scale for P-4. Nevertheless, because the index score for P-4 is qualified in terms of its validity due to the issue of overcounting "other" references, this finding is questionable. This finding needs further substantiation. Chances are that further evidence supporting this proposition would be found. This is because the locus-of-control regarding foreign policy decisions lies with foreign policymakers and other important external actors. It lies with "others" and not "self."

Beyond support for $\mathrm{H}-4$ and $\mathrm{H}-6$, the results of the data analyses provide mixed support for H-1 and H-2 and no support for H-3 and H-5. The index score for H-1 (0.16), which posits that U.S. intelligence has a hostile image of the political universe and the fundamental nature of their political opponents, is anchored to the descriptor "Mixed" on the nature of the political universe scale for P-1. Similarly, for my hypothesis that U.S. intelligence has an optimistic view 
regarding the realization of its fundamental political values, the index score of 0.08 falls within the category of "Mixed" on the realization of political values scale for P-2. These findings provide some support for $\mathrm{H}-1$ and $\mathrm{H}-2$, but by no means solid or even tentative support. The index score for H-3 (0.06) is anchored to the descriptor "Very Low" on the predictability of the political future scale for P-3, and the index score for H-5 (0.99) is anchored to the descriptor "High" on the role of chance scale for P-5. These data provide no support for H-3 and H-5. The former argues that the IC believes the political future is predictable and the latter posits that U.S. intelligence attributes a low role to chance.

What does all this mean for my theory that that the core beliefs that the IC holds about world politics underlie several U.S. intelligence norms? Even though there was a lack of evidence supporting $\mathrm{H}-1, \mathrm{H}-2, \mathrm{H}-3$, and $\mathrm{H}-5$, I do not believe that this dissertation's theory should be completely scrapped. For one, there was evidence supporting H-4, and H-1 and H-2 were partially supported. These findings indicate that the theory was not completely wrong. Another reason why my theory should not be rejected outright is that research on U.S. intelligence norms is still a relatively small body of scholarship within intelligence studies that needs more attention. If more research was available, my hypotheses would be more precise. Another reason why my theory warrants further examination is that the speech act may have been inappropriate for calculating the philosophical indices. However, I was more or less forced to employ NIEs as my speech act since NIEs are the only publically available source that could reflect and aggregate the IC's worldview with reasonable accuracy. Over time, other sources might become available that would do a better job of capturing the worldview of U.S. intelligence. Finally, other types of content analysis beyond VICS could deny the findings presented in this chapter. There is no guarantee that this subsequent content analyses would 
agree with this dissertation's theory and hypotheses, but this will only be known if further analyses are conducted.

\section{Chapter Summary}

This chapter began by presenting VICS data on the philosophical beliefs in an operational code. These data were obtained from verb-based utterances in thirty declassified National Intelligence Estimates. The mean values demonstrated that the IC has a mixed view about the nature of the political universe (friendly/hostile) and about the realization of their political values (optimistic/pessimistic), it believes that the chance of accurately predicting the political future is very low, it has very little control over historical development, and it attributes a very high role to chance. The second section presented the results of two ANOVA tests (Kruskal-Wallis and Welch). These data strongly suggested that there are no statistically significant differences in the philosophical index scores between the six subgroups identified in the previous chapter. This confirmed my proposition that the worldview of U.S. intelligence is specific and longstanding was confirmed. Section three discussed what these data analyses mean for this dissertation's theory and hypotheses. The major claim made in this section was that despite limited evidence supporting the hypotheses presented in chapter three, this dissertation's theory warrants further examination. The next (and final) chapter presents this dissertation's conclusions and delineates future research suggestions. 


\section{CHAPTER SIX}

\section{GENERAL CONCLUSIONS: FUTURE RESEARCH ON THE COGNITIVE CULTURE OF U.S. INTELLIGENCE}

This dissertation began with the stated purpose of determining what the worldview of U.S. intelligence (its cognitive culture) looks like so that it can be incorporated into America's intelligence identity. I argued that this was necessary to gain a better understanding of why the agencies that comprise the IC have shared behaviors despite having different interests and preferences. To answer my research question, which asked what the worldview of U.S. intelligence is, I conceptualized the IC's core belief system about world politics (its worldview) as the set of philosophical beliefs in an operational code. This set includes five cognitions. These are the nature of the political universe, prospects for realizing fundamental political values, predictability of the political universe, control over historical development, and the role of chance. I hypothesized about how the IC views each of these beliefs in accordance with my theory that the IC's worldview underlies several U.S. intelligence norms. I also posited that the worldview of U.S. intelligence is specific and longstanding. However, after testing my hypotheses using automated content analysis and statistical methods, I only found strong support for two of my six propositions.

What are this dissertation's general conclusions? Although my analysis did not produce a definitive answer to my research question, one conclusion is that the core beliefs that U.S. intelligence holds about world politics warrant further study. As I explained in the first chapter, cognitions underlie all political behavior and form the foundation for how power and interests are understood (Young and Schafer 1998, 84). Therefore, constructivism, which is at base cognitive rather than behavioral, has to be enriched with insights about cognition (Wendt 1999, 
394). Still, almost no constructivist scholars, including those writing about intelligence, have followed this recommendation so far. In turn, a glaring set of cognitions that has been missing from the U.S. intelligence identity is the IC's worldview. This is problematic since it prevents constructivists writing about intelligence from being able to fully account for shared behaviors related to foreign intelligence activities. Future research on the IC's worldview is necessary to fill in this gap in the literature.

Another conclusion is that this dissertation's findings are a useful starting point for those trying to characterize the IC's core belief system about world politics. This modest contribution provides researchers with a list to check their own findings against, which is at the heart of social science research. Indeed, "there is no such thing as the perfect experiment that explains everything about a given phenomena" (Hoover and Donovan 2001, 59). Rather, studies are supposed to build upon one another so that a large body of knowledge on a particular subject can be accumulated. It is entirely likely that subsequent studies will produce further denying my findings, as well as my theory and hypotheses even further. However, these projects might not have been undertaken in the first place if this dissertation had not laid the groundwork for future research on the IC's cognitive culture.

Finally, since data analysis provided strong support for my hypothesis on the continuity of the IC's worldview, I conclude that the worldview of U.S. intelligence is in all likelihood specific and longstanding. That is, it is well-established and continuous. This finding challenges the general belief within operational code literature that the five philosophical beliefs are especially prone to fluctuation by domain over time in responses to changes in context (Schafer and Crichlow 2000; Walker, Schafer, and Young 1998). This is not surprising, however. Core beliefs are supposed to be absolutistic and rigid (Beck 1995; Jervis 1976; Nisbett and Ross 
1980), especially when it comes to organizations (Cameron and Quinn 1999; Hatch 2004; Schein 1996, 2004). What this finding ultimately suggests is that IC's worldview is and always has been a part of its raison d'etre. I believe that future research will confirm this finding. The reasons for this were discussed in chapter five.

Even though I believe that this dissertation is an important addition to the literature on intelligence, albeit a modest one, its fundamental value is its ability to spark a new research agenda. To facilitate a research program that focuses on the worldview of U.S. intelligence, the remainder of this chapter does two things. First, it discusses three ways to go about doing more research on what the worldview of U.S. intelligence looks like. Second, it explains the various research implications of a research program within intelligence studies that focuses on the IC's cognitive culture. I end with a chapter summary and make some concluding remarks.

\section{Future Research Strategies}

There are several research methodologies available within the social sciences than can be utilized for those researching the IC's core belief system about world politics. However, some are likely to be more useful than others. In this section, I discuss what some of these methodological approaches are and how they can be used to generate the IC's worldview approaches. The discussion of each approach will be brief, but enough information is provided so that researchers can investigate the methodologies further. I should point out that the list of methodological approaches I discuss is by no means exhaustive. Nor do I advocate one approach over another. This list is simply a delineation of the most salient approaches that come to mind. It is my hope that scholars undertaking a cognitive research agenda within intelligence studies will employ my suggestions so that more empirical research on the IC's worldview is generated. I also hope that researchers produce new and innovative strategies for tackling the question of 
what the worldview of U.S. intelligence is.

The first approach I recommend is case study analysis. Case studies, which are a form of qualitative descriptive research, were considered the primary research tool in the social sciences until the development of the scientific method. A case study is defined as an empirical inquiry that relies on multiple sources of evidence to investigate a phenomenon within its real-life context (Yin 2002). Tt is a systematic way of looking at events, collecting data, analyzing data, and reporting the results. Rather than using large samples and following a rigid protocol to examine a limited number of variables, case study analysis involves an in-depth, longitudinal examination of a single instance or event: a case. Case studies produce highly detailed analyses that are rich in contextual information, creative, and innovative.

Case studies do not focus on the discovery of a generalizable truth, nor do they typically look for cause-effect relationships. Instead, they emphasize exploration and description. This gives intelligence studies scholars the ability to gain a sharper understanding of the cognitions that comprise the worldview of U.S. intelligence. Indeed, what is particularly useful about case studies is they lend themselves to both generating and testing hypotheses (Flyvbjerg 2006). On the one hand, case study analysis can be used by researchers to generate hypotheses on what the worldview of U.S. intelligence looks like (Eisenhardt 1989, 532-550). This type of case study should be organized using some form of framework and serve as a pilot study for more sustained study of the case (Berg 2004, 255-257). Researchers could use the operational code framework that I utilized in this dissertation, use a different cognitive construct, or examine a long list of beliefs and eliminate those that are not supported by the evidence. On the other hand, case study analysis' descriptive side could be used to re-test the hypotheses that I presented in this dissertation or to test new ones. Regardless of the type of case study undertaken, analysis of the 
IC's worldview should involve the comparison of different time periods. ${ }^{1}$ This tests my finding that the worldview of U.S. intelligence is specific and longstanding.

The second approach I recommend is content analysis. Content analysis is a research tool used to determine the presence of certain words or concepts within texts or sets of texts (Neuendorf 2002). Researchers quantify and analyze the presence, meanings, and relationships of such words and concepts, then make inferences about the messages within the texts, the writer(s), the audience, and even the culture and time of which these are a part. Texts can include books, book chapters, essays, interviews, discussions, newspaper headlines and articles, historical documents, speeches, conversations, informal conversation, or any other occurrence of communicative language. To conduct a content analysis, the text is broken down (coded) into manageable categories on a variety of levels and then examined using one of content analysis' basic methods.

The content analysis scheme that this dissertation employed is just one of many that could be used to extrapolate the IC's core belief system about world politics. Granted, as I explained in chapter four, it is inappropriate to compare VICS dated coded by a computer to that which is coded by hand or other processes. However, this is not to say that approaches outside of automated coding with VICS are worthless. Various forms of content analysis can be used to generate new hypotheses that can then be tested via case study analysis or through statistical techniques. I suggest that further automated operational code analysis using VICS be done first on other types of data sources, such as DCI statements, and then compared with the results of this study. This is because I believe that the operational code approach is an extremely useful research technique within the field of cognition. It should employed to the point of exhaustion.

\footnotetext{
${ }^{1}$ The literature on this method of inquiry is vast. For a good overview of the literature see Lijphart (1971) and Collier (1993).
} 
This approach will become easier over time as more and more IC documents are declassified, including NIEs.

The third and final approach I recommend is survey research. Surveys are one of the most common types of quantitative, social science research. In survey research, the analyst selects a sample of respondents from a population and administers a standardized questionnaire to them. The questionnaire, or survey, can be a written document that is completed by the person being surveyed, an online questionnaire, a face-to-face interview, or a telephone interview. Surveys have several advantages. They are an efficient way of collecting information from a large number of respondents, they are flexible in the sense that a wide range of information can be collected, they are relatively free from several types of errors, and they are relatively easy to administer (Ornstein 1998).

The major advantage that surveys provide for intelligence scholars is that they allow give them the ability to gather multiple points of view from current and former IC employees. However, conducting interviews with current and former intelligence officials is by no means an easy task due to the secretive nature of the intelligence business. To help overcome this, I recommend that researchers employ the Delphi Method of survey research when investigating the IC's core belief system about world politics. The Delphi Method is a multiple iteration survey technique used to provide refined estimates of opinion while allowing for a high degree of informational exchange across a panel of experts (Ziglio 2002). ${ }^{2}$ This method allows the panel to provide input and allows the researcher to avoid questionable omissions associated with instrument design. In addition, the method allows the researcher to develop refined estimates of opinion across various panel backgrounds in order to control for potential bias. Implementation

\footnotetext{
${ }^{2}$ In the case of the IC's worldview, the panel of experts should include academics, current and former intelligence practitioners, and independent experts.
} 
of the Delphi Method consists of multiple iterations of a panel survey in which the members are isolated from each other. Frequently, an initial round, or zero round, is conducted to provide a baseline for subsequent rounds and to insure that the researchers have accurately accounted for important factors. Each iteration of the survey presents feedback from the previous rounds to test for convergence among expert opinion. The use of open-ended questions is common as well. This prevents groupthink from occurring, while allowing the researcher to gather better understanding of the case using the repeated panel responses.

\section{Future Research Implications}

The primary reason why this dissertation attempted to characterize the worldview of U.S. intelligence was to incorporate it into America's intelligence identity to gain a better understanding of why the various agencies within the IC act similarly in several ways. However, the potential benefits that can come from determining and analyzing the IC's cognitive culture are vast. In this section, I discuss five future research implications. These suggestions focus on some of the most important research areas within the discipline of intelligence studies, such as intelligence failure and intelligence reform. Once again, this list is by no means exhaustive. Rather, it is representative of what I consider the most pronounced benefits of continued research on the worldview of U.S. intelligence.

One potential future research implication is that knowing the exact cognitions that comprise the IC's worldview can help researchers generate further insight in the cognitive causes of intelligence failure. As Richards J. Heuer Jr. (2005) explains, "of the diverse problems that impede accurate and timely intelligence analysis and contribute to intelligence failure, problems inherent to mental processes are surely among the most important and most difficult to remedy" (78). This is because limits in mental capacity limit the mind's ability to cope directly with the 
complexity of the modern world (Simon 1957). To overcome this, intelligence analysts construct a simplified mental reality of reality, including a worldview, and then work with this model (Heuer 2005, 78). Researchers generally agree that intelligence failures most often occur because "available information — or the absence of information—was discounted, misinterpreted, ignored, rejected, or overlooked because it failed to fit a prevailing mental model or mindset that influenced the analyst's perception and interpretation of the evidence" (Hauer 2005, 84). Given that intelligence analysts internalize the IC's worldview due to socialization into the IC (Halperin 1974; Heuer 1999; Immerman 2008; Jackson and Siegel 2005; Johnston 2005; Wirtz 1991), the worldview of U.S. intelligence can be used as a checklist by researchers attempting to identify the misperceptions and misinterpretations that caused particular intelligence failures.

A second and related implication is that identifying the exact cognitions that comprise the IC's worldview might highlight the reforms that are most useful for preventing intelligence failure. Most often, proposals for intelligence reform focus on relations between intelligence analysts and intelligence consumers, types of analytical products, organization of the analytical process, the quality of writing, or providing language and area studies to improve substantive expertise (Heuer 2005, 89-90). Only rarely do intelligence reform proposals focus on how analysts think and comparing this with a more sophisticated ideal. This is unfortunate given that

to penetrate to the heart and soul of the problem of improving intelligence analysis, it is necessary to better understand, influence, and guide the mental process of intelligence analysts. To reduce the risk of surprise in a rapidly changing world, intelligence analysts need to develop habits of mind and use analytical tools that help them become the first to recognize that old assumptions may no longer apply. They must learn to recognize when underlying conditions have changed so much that their mindset, which has served them so well in the past, may no longer be appropriate. On a personal level, they need to learn how to recognize when to change their minds. (Ibid., 90)

I believe that knowing the exact cognitions that comprise the IC's core belief system about world politics would help researchers propose reforms that suggest ways to combat antiquated beliefs. 
A third future research implication is that it could help researchers identify hard to detect intelligence norms. Indeed, even if this dissertation's hypotheses are discredited further by future research, this does not necessarily mean that the IC's worldview is not a part of its identity. It might just mean that the norms these cognitions underlie are not present in the current literature. This would be a consequence of that the fact that constructivists writing about intelligence have ignored the IC's worldview when constructing the U.S. intelligence identity. Extrapolating the IC's core belief system about world politics could spawn intelligence norms that were not easily apparent to scholars.

A fourth future research implication is that characterizing the IC's worldview will allow researchers to explore the psychological foundation for the norm of intelligence exceptionalism. This norm, which I discussed in detail in chapter three, suggests that intelligence is different and more important than other governmental activities. Although several intelligence scholars have investigated how the IC is different from other government agencies, especially from those in the national security and foreign policy communities, no scholar has examined its cognitive underpinning. Researchers could compare the IC's core belief system about world politics to that of other governmental organizations. Numerous scholars have already conducted research on the foreign belief systems of national security and foreign policy agencies, including the military and the Foreign Service. ${ }^{3} \quad$ Moreover, researchers have a wealth of organizational culture literature available to them for extrapolating, identifying, and refining the worldviews of various governmental organizations.

Finally, a fifth implication is that extrapolating the IC's worldview will allow researchers to open up a new research program within comparative intelligence studies on cognitive culture.

\footnotetext{
${ }^{3}$ For research on the former see Adams (1990), Builder (1989), Breslin (2000), and Dunivin (1997). See Steward (2006) for research on the latter.
} 
For example, researchers could see if the IC's worldview is distinct from that of other advanced democracies. This could help validate, or invalidate, the claim made by several constructivists writing about intelligence that even though U.S. intelligence is not fundamentally unique, its identity distinguishes it from that of other advanced democracies (Sims 2005; Turner 2004, 2006; Wark 2003). Identifying just how unique the IC's core belief system about world politics is could also generate new insight into why the intelligence services of advanced democratic societies operate the way that they do. All types of organizations have some sort of a belief system that provides a core set of values, which forms the basis for their goals and priorities. Understanding the worldviews of foreign intelligence communities, including those of nonadvanced democracies, could also help intelligence scholars identify a general theory of intelligence. Intelligence cannot be fully understood without a good grasp of the theory behind what intelligence is or should be about (Sims 2005). I believe that understanding the fundamental assumptions that intelligence communities generally make to achieve their missions would facilitate the work of scholars trying to elucidate a general theory of intelligence.

\section{Chapter Summary and Concluding Remarks}

This chapter began by presenting this dissertation's overall conclusions. My first conclusion was that the core beliefs that U.S. intelligence holds about world politics warrant further study. This is necessary to fill in the literature gap identified in chapter one. A second conclusion was that this dissertation's findings are a useful starting point for those trying to characterize the IC's core belief system about world politics. Finally, my third conclusion was that the worldview of U.S. intelligence is in all likelihood specific and longstanding. That is, the IC's core beliefs about world politics are more than likely not prone to fluctuation over time in responses to changes in context. After discussing these conclusions, I suggested three methods 
that intelligence scholars could use to further characterize the IC's worldview. These were case study analysis, content analysis, and survey research. This section was succeeded by one that discussed several potential future research implications.

Even though this dissertation's analysis did not provide a definitive answer to its research question, I believe that I have convincingly demonstrated the importance of a research agenda within intelligence studies that focuses on the IC's cognitive culture. I have no doubt that future research on this topic would generate theoretical and practical benefits. Several of these potential benefits were discussed in this chapter. Moreover, I hope that dissertation will serve as a key reference to those researchers who undertake my research suggestions. In the end, the ultimate value of this dissertation lies not in what its analysis found, but rather in the fact that it is first study to empirically analyze the set of core cognitions that the U.S. intelligence holds about foreign affairs and global issues among state and non-state actors (world politics). 


\section{BIBLIOGRAPHY}

Abelson, Robert P. and Milton J. Rosenberg. 1958. "Symbolic Psychologic: A Model of Attitudinal Cognition." Behavioral Science 3: 1-13.

Adams, Thomas K. 1990. "Doctrine and the Organizational Culture of the U.S. Army." Ph.D. Dissertation. Syracuse, NY: Syracuse University.

Allison, Graham. 1969. "Conceptual Models and the Cuban Missile Crisis." American Political Science Review 63: 689-718.

Allison, Graham. 1971. Essence of Decision. Boston, MA: Little Brown.

Allison, Graham and Morton H. Halperin. 1972. "Bureaucratic Politics: A Paradigm and Some Policy Implications.” World Politics 24: 40-79.

Andrew, Christopher. 1994. "Intelligence, International Relations and 'Under-theorisation'." Intelligence and National Security 19.2: 170-184.

Andrew, Christopher. 1995. For the Presidents Eyes Only: Secret Intelligence and the American Presidency from Washington to Bush. New York, NY: Harper Collins.

Art, Robert J. 1973. "Bureaucratic Politics and Foreign Policy: A Critique." Policy Sciences 4: 467-490.

Aspin-Brown Commission. 2004. "The Evolution of the U.S. Intelligence Community-An Historical Overview." In Strategic Intelligence: Windows into a Secret World, edited by Jim Wirtz and Loch Johnson. Los Angeles, CA: Roxbury Publishing Company.

Austin, James D. 1985. “The Psychological Dimension of Intelligence Activities." In Intelligence Policy \& Process, edited by Alfred C. Maurer, Marion D. Tunstall, and James M. Keagle. Boulder, CO: Westview Press.

Axelrod, Robert. 1972. "Psycho-Algebra: A Mathematical Theory of Cognition with Choice with an Application to the British Eastern Committee in 1918." Papers of the Peace Research Society 18: 113-131.

Axelrod, Robert. 1976a. "The Analysis of Cognitive Maps." In Structure of Decision, edited by Robert Axelrod. Princeton, NJ: Princeton University Press.

Axlerod, Robert, ed. 1976b. Structure of Decision. Princeton, NJ: Princeton University Press.

Bass, Bernard M. 1981. Stogdill's Handbook of Leadership. New York, NY: Free Press.

Beck, Judith S. 1995. Cognitive Therapy: Basics and Beyond. New York, NY: Guilford. 
Berg, Bruce. 2004. Qualitative Research Methods for the Social Sciences. New York, NY: Pearson, Allyn, and Bacon Publishers.

Betts, Richard. 1978. "Analysis, War, and Decision: Why Intelligence Failures Are Inevitable." World Politics 31.1: 61-89.

Betts, Richard. 2002. "Fixing Intelligence." Foreign Affairs 81.1: 43-59.

Blalock, Herbert M. 1964. "Cognitive Mapping as a Technique for Supporting International Negotiation." Theory and Decision 34: 255-273.

Bonham, G. Matthew and Michael J. Shapiro. 1976. "Explanation of the Unexpected: The Syrian Intervention in Jordan in 1970." In Structure of Decision, edited by Robert Axelrod. Princeton, NJ: Princeton University Press.

Borhek, James T. and Curtis, Richard F. 1975. A Sociology of Belief. New York, NY: John Wiley \& Sons.

Boulding, Kenneth E. 1956. The Image. Ann Arbor, MI: University of Michigan Press.

Boulding, Kenneth E. 1959. "National Images and International Systems." Journal of Conflict Resolution 3: 120-131.

Brady, Henry E. and David Collier. 2004. Rethinking Social Inquiry. New York, NY: Rowman and Littlefield Publishers.

Breslin, Charles B. 2000. "Organizational Culture and the Military." Carlisle, PA: Army War College: http://handle.dtic.mil/100.2/ADA378263.

Bruno, Greg and Sharon Otterman. 2008. National Intelligence Estimates. New York, NY: Council on Foreign Relations.

Bueno De Mesquita, Bruce and David Lalman. 1986. War and Reason. New Haven, CT: Yale University Press.

Builder, Charles. 1989. The Masks of War: American Military Styles in Strategy and Analysts. Baltimore, MD: Johns Hopkins University Press.

Byars, Robert S. 1973. "Small-Group Theory and Shifting Styles of Political Leadership." Comparative Political Studies 5:443-469.

Cartwright, Dorwin and Frank Harary. 1965. "Structural Balance: A Generalization of Heider's Theory." Psychological Review 63: 277-293.

Casey, William J. 1989. Scouting the Future: The Public Speeches of William J. Casey. Houston, TX: Regency Publishing. 
Cameron, Kim S. and Robert E. Quinn. 1999. Diagnosing and Changing Organizational Culture: Based on the Competing Values Framework. Upper Saddle River, NJ: Prentice Hall.

Cimbala, Stephen J. 1988. “Amorphous Wars." International Journal of Intelligence and Counterintelligence 2.1: 60-80.

Clark, Robert M. 2004. Intelligence Analysis: A Target-Centric Approach. Washington, DC: CQ Press.

Collier, David. 1993. "The Comparative Method." In Political Science: The State of the Discipline II, edited by Ada W. Finifter. Washington, DC: American Political Science Association.

Converse, Philip. 1964. "The Nature of Belief Systems in Mass Publics." In Ideology and Discontent, edited by David Apter. London, England, UK: Free Press of Glencoe.

Cottam, Martha. 1986. Foreign Policy Decision Making: The Influence of Cognition. Boulder, CO: Westview Press.

Cottam, Martha. 1992. “ The Carter Administration's Policy Toward Nicaruaga: Images, Goals, and Tactics." Political Science Quarterly 107: 123-146.

Cottam, Richard. 1977. Foreign Policy Motivation: A General Theory and a Case Study. Pittsburgh, PA: University of Pittsburgh Press.

Crichlow, Scott. 1998. "Idealism or Pragmatism? An Operational Code Analysis of Yitzhak Rabin and Shimon Perez." Political Psychology 16.4: 683-706.

Crichlow, Scott. 2005. "Psychological Influences on the Policy Choices of Secretaries of State and Foreign Ministers." Conflict and Cooperation 40.2: 179-205.

Davies, Philip H. J. 2004. "Intelligence Culture and Intelligence Failure in Britain and the United States." Cambridge Review of International Affairs 17.3: 495-520.

Dawson, Paul A. 1979. "The Formation and Structure of Belief Systems." Political Behavior 1.2: $99-122$.

Downs, Roger G. and David Stea. 1973. Image and the Environment: Cognitive Mapping and Spatial Behavior. Piscataway, NJ; Transaction Publishers.

Driver, Michael J. 1977. "Individual Differences as Determinants of Aggression in the Inter -Nation Simulation." In A Psychological Examination of Political Leaders, edited by Margaret G. Hermann. New York, NY: Free Press. 
Druckman, Daniel. 1968. "Ethnocentrism and the Inter-Nation Simulation." Journal of Conflict Resolution 12: 45-68.

Drury, A. Cooper. 2000. "U.S. Presidents and the Use of Economic Sanctions." Presidential Studies Quarterly 30: 623-642.

Dunivin, Karen O. 1997. “Military Culture: A Paradigm Shift?” Maxwell Air Force Base, AL: Air War College: http://handle.dtic.mil/100.2/ADA418457.

Eisenhardt, Kathleen M. 1989. "Building Theories from Case Study Research." The Academy of Management Review 14.4: 532-550.

Feng, Huiyan. 2006. "Crisis Deferred: An Operational Code Analysis of Chinese Leaders Across the Strait." In Beliefs and Leadership in World Politics: Methods and Applications of Operational Code Analysis, edited by Mark Schafer and Stephen G. Walker. New York, NY: Palgrave Macmillan.

Finnemore, Martha. 1996. National Interests in International Society. Ithaca, NY: Cornell University Press.

Flyvjberg, Bent. 2001. Making Social Science Matter: Why Social Inquiry Fails and How It Can Succeed Again. Cambridge, MA: Cambridge University Press.

Ford, Harold P. 1993. "The Primary Purpose of National Estimating." In Estimative Intelligence: The Purposes and Problems of National Intelligence Estimating, edited by Harold P. Ford. Lanham, MD: University Press of America.

Garthoff, Douglas F. 2005. Directors of Central Intelligence as Leaders of the U.S. Intelligence Community. Washington, DC: Central Intelligence Agency.

George, Alexander. 1969. "The 'Operational Code': A Neglected Approach to the Study of Political Leaders and Decision-Making.” International Studies Quarterly 13: 190-122.

George, Alexander. 1979. "The Causal Nexus Between Beliefs and Behavior." In Psychological Models in International Politics, edited by Lawrence Falkowski. Boulder, CO; Westview Press.

George, Alexander. 1980. Presidential Decision Making in Foreign Policy: The Effective Use of Information and Advice. Boulder, CO: Westview Press.

George, Alexander L. and Timothy J. McKeown. 1985. "Case Studies and Theories of Organizational Decision Making." Advances in Information Processing Organizations 2: 21-58.

Giddens, Anthony. 1984. The Constitution of Society. Berkeley, CA: University of California Press. 
Gill, Peter. 1994. Policing Politics: Security Intelligence and Liberal Democratic State. Portland, MA: Frank Cass.

Goldgeier, James M. 1997. "Psychology and Security.” Security Studies 6: 137-166.

Gossett, William S. 1908. “The Probable Error of a Mean.” Biometrika 6.1: 1-25.

Haley, Edward P. 1989. "Legislative-Executive Relations and the United States Intelligence Community." Harvard Journal of Law and Public Policy 12: 102-166.

Halperin, Morton. 1974. Bureaucratic Politics and Foreign Policy. Washington, DC: Brookings.

Hamilton, Mark E. and Paul J. Mineo. 1996. "Personality and Persuasibility: Developing a Multidimensional Model of Belief Systems." World Communication 24: 1-16

Hamilton, Mark E. and Paul J. Mineo. 1998. "A Framework for Understanding Equivocation." Journal of Language and Social Psychology 17: 3-35.

Handel, Michael I. 1987. "The Politics of Intelligence." Intelligence and National Security 4.2: $5-46$.

Handel, Michael I. 1988. "Leaders and Intelligence." Intelligence and National Security 3.3: 3-39.

Harary, Frank, Robert Z. Norman, and Dorwin Cartwright. 1965. Structural Theory: An Introduction to the Theory of Directed Graphs. New York, NY: John Wiley and Sons.

Hart, Jeffrey. 1976. "Comparative Cognition: Politics of International Control of the Oceans." In Structure of Decision, edited by Robert Axelrod. Princeton, NJ: Princeton University Press.

Hastedt, Glenn P. 1985. “Organizational Foundations of Intelligence Failures.” In Intelligence Policy \& Process, edited by Alfred C. Maurer, Marion D. Tunstall, and James M. Keagle. Boulder, CO: Westview Press.

Hatch, Mary Jo. 2004. "Dynamics in Organizational Culture." In Handbook of Organizational Change and Innovation, edited by Marshall Scott Poole and Andrew H. Van de Ven. New York, NY: Oxford University Press.

Helms, Richard, William Hood, and Henry Kissinger. 2003. A Look Over My Shoulder: A Life in the Central Intelligence Agency. New York, NY: Random House.

Hemmer, Christopher. 1999. "Historical Analogies and the Definition of Interests: The Iranian Hostage Crisis and Ronald Reagan's Policy Toward Hostages in Lebanon." Political Psychology 20.2: 267-289. 
Herman, Michael. 1996. Intelligence in Peace and War. New York, NY: Cambridge University Press.

Hermann, Margaret G. 1977. A Psychological Examination of Political Leaders. New York, NY: Free Press.

Hermann, Margaret G. 1980. "Explaining Foreign Policy Behavior Using the Personal Characteristics of Political Leaders." International Studies Quarterly 24: 7-46.

Hermann, Margaret G. 1984 "Personality and Foreign Policy Making." In Perceptions, Beliefs, and Foreign Policy Decision Making, edited by Donald Sylvan and Steve Chan. New York, NY: Praeger.

Hermann, Margaret G. 1985. "Validating a Technique for Assessing Personalities of Political Leaders at a Distance: A Test Using Three Heads of State." Report prepared for Defense Systems, Inc. for Contract DSI-84-1240.

Hermann, Margaret G. 2001. "How Decision Units Shape Foreign Policy: A Theoretical Framework." International Studies Review 3.2: 47-81.

Hermann, Margaret G. 2005. "Assessing Leadership Style: A Trait Analysis." In The Psychological Assessment of Political Leaders, edited by Jerrold M. Post. Ann Arbor, MI: The University of Michigan Press.

Hermann, Margaret G. and Hermann, Charles F. 1989. "Who Makes Foreign Policy Decisions and How: An Empirical Inquiry." International Studies Quarterly 33: 361-387.

Hermann, Margaret G. and Nathan Kogan. 1977. "Personality and Negotiating Behavior." In Negotiations: Social-Psychological Perspectives, edited by Daniel Druckman. Beverly Hills, CA: Sage Publications.

Hermann, Margaret G. and Preston, Thomas. 1994. "Presidents, Advisers, and Foreign Policy: The Effect of Leadership Style on Executive Arrangements." Political Psychology 15: $5-96$.

Herrmann, Richard K. 1984. "Perceptions and Foreign Policy Analysis." In Foreign Policy Decision Making: Perception, Cognition, and Artificial Intelligence, edited by Donald Sylvan and Steve Chan. New York, NY: Praeger.

Herrmann, Richard K. 1985. Perceptions and Behavior in Soviet Foreign Policy. Pittsburgh, PA: University of Pittsburgh Press.

Herrmann, Richard K. 2003. "Image Theory and Strategic Interaction in International Relations." In Oxford Handbook of Political Psychology, edited by David O. Sears, Leonie Huddy, and Robert Jervis. New York, NY: Oxford University Press. 
Herrmann Richard K., James F. Voss, Tonya Y.E. Schooler, and Joseph Ciarrochi. 1997. "Images in International Relations: An Experimental Test of Cognitive Schemata." International Studies Quarterly 41: 403-433.

Heuer, Richards J., Jr. 1999. Psychology of Intelligence Analysis. Washington, DC: Government Printing Office.

Heuer, Richards J., Jr. 2005. “Limits of Intelligence Analysis.” Orbis Winter: 75-94.

Hilsman, Roger. 1958. Strategic Intelligence and National Decisions. Glencoe, IL: Free Press.

Holsti, Ole. 1967. "Cognitive Dynamics and Images of the Enemy." Journal of International Affairs. 21: 16-39.

Holsti, Ole. 1977. "The 'Operational Code' as an Approach to the Analysis of Belief Systems." The Final Report to the National Science Foundation, Grant SOC 75-16368: Duke University.

Hoover, Kenneth and Todd Donovan. 2001. The Elements of Social Scientific Thinking. Boston, MA: St. Martins.

Hopf, Ted. 1994. Peripheral Visions: Deterrence Theory and American Foreign Policy in the Third World. Ann Arbor, MI: University of Michigan Press.

Hopf, Ted. 2002. Social Construction of International Politics: Identities and Foreign Policies, Moscow, 1955 and 1999. Ithaca, NY: Cornell University Press.

Hudson, Valerie M. 2005. "Foreign Policy Analysis: Actor-Specific Theory and the Ground of International Relations." Foreign Policy Analysis 1: 1-30.

Hudson, Valerie M. 2006. Foreign Policy Analysis: Classic and Contemporary Theory. Lanham, MD: Rowman and Littlefield.

Hughes, Thomas L. 1976. The Fate of Fact in Men: Foreign Policy and Intelligence-Making. New York, NY: Foreign Policy Association.

Hulnick, Arthur S. and David W. Mattausch. 1989. "Ethics and Morality in United States Intelligence." Harvard Journal of Law and Public Policy 12: 509-522.

Huntington, Samuel P. 1960. "Strategic Planning and the Political Process." Foreign Affairs 38: 285-299.

Immerman, Richard H. 2008. "Intelligence and Strategy: Historicizing Psychology, Policy, and Politics." Diplomatic History 32.2: 1-23. 
Jackson, Peter J. and Jennifer L. Siegel, eds. 2005. Intelligence and Statecraft: The Uses and Limits of Intelligence in International Society. Dulles, VA: Brassey’s Inc.

Janis, Irving L. 1982. Groupthink: Psychological Studies of Policy Decisions and Fiascoes. New York, NY: Houghton Mills.

Jervis, Robert. 1976. Perception and Misperception in International Relations. Princeton, NJ: Princeton University Press.

Jervis, Robert. 1985. "Improving the Intelligence Process: Informal Norms and Incentives." In Intelligence Policy \& Process, edited by Alfred C. Maurer, Marion D. Tunstall, and James M. Keagle. Boulder, CO: Westview Press.

Johnson, Loch K. 1977. "Operational Codes and the Prediction of Leadership Behavior: Senator Frank Church at Midcareer." In A Psychological Examination of Political Leaders, edited by Margaret G. Hermann. New York, NY: Free Press.

Johnson, Loch K. and James J. Wirtz, eds. 2004. Strategic Intelligence. Los Angeles, CA: Roxbury Publishing Company.

Johnston, Alastair Iain. 1996. "Cultural Realism and Strategy in Maoist China." In The Culture of National Security: Norms and Identity in World Politics, edited by Peter J. Katzenstein. New York, NY: Columbia University Press.

Johnston, Robert. 2005. Analytic Culture in the US Intelligence Community: An Ethnographic Study. Washington, DC: Government Printing Office.

Jordan, Amos A., William T. Taylor Jr., and Michael J. Mazarr. 1999. American National Security. Baltimore, MD: Johns Hopkins University Press.

Kaplowitz, Stan. 1978. "Towards a Systematic Theory of Power Attribution.” Social Psychology 41: 131-148.

Karbo, Juliet and Margaret G. Hermann. 1998. "Leadership Styles of Prime Ministers: How Individual Differences Affect the Foreign Policymaking Process." Leadership Quarterly 9: 243-263.

Katzenstein, Peter J., ed. 1996. The Culture of National Security: Norms and Identity in World Politics. New York, NY: Columbia University Press.

Katzenstein, Peter J. 1998. Cultural Norms and National Security: Police and Military in Postwar Japan. Ithaca, NY: Cornell University Press.

Keagle, James M., Marion D. Tunstall, and Alfred C. Maurer, eds. 1985. Intelligence Policy \& Process. Boulder, CO: Westview Press. 
Kent, Sherman. 1949. Strategic Intelligence for American Foreign Policy. Princeton, NJ: Princeton University Press.

Kent, Sherman. 1964. "Words of Estimative Probability." Studies in Intelligence 8.4: 49-65.

Kesselman, Rachel F. 2008. Verbal Probability Expressions in National Intelligence Estimates: A Comprehensive Analysis from the Fifties Through Post 9/11. M.A. Thesis. Erie, PA: Mercyhurst College.

Kim, Wonik and Bruce Bueno De Mesquita. 1995. "How Perceptions Influence the Risk of War.” International Studies Quarterly 39.1: 51-65.

Kinzer, Stephen. 2003. All the Shah's Men: An American Coup and the Roots of Middle East Terror. New York, NY: John Wiley and Sons.

Kitchin, Robert M. 1994. “Cognitive Maps: What Are They and Why Study Them?” Journal of Environmental Psychology 14: 1-19.

Koltko-Rivera, Mark E. 2004. "The Psychology of Worldviews." Review of General Psychology 8.1: 3-58.

Krasner, Stephen. 1972. “Are Bureaucracies Important?” Foreign Policy 7.3: 159-179.

Kubalkova, Vendulka, ed. 2001. Foreign Policy in a Constructed World. Armonk, NY: M.E. Sharpe.

Kuhns, Woodrow. 2007. "The Office of Reports and Estimates: CIAs First Center for Analysis." Studies in Intelligence 51..2: 1-20.

Lake, David and Robert Powell. 1999. Strategic Choice and International Relations. Princeton, NJ: Princeton University Press.

Langer, Ellen J. 1983. The Psychology of Conflict. Beverly Hills, CA: Sage Publications.

Laqueur, Walter. 1985. A World of Secrets: The Uses and Limits of Intelligence. New York, NY: Basic Books.

Lefcourt, H. M. 1976. Locus of Control: Current Trends in Theory and Research. New York, NY: Halstead.

Leites, Nathan. 1951. The Operational Code of the Politburo. New York, NY: McGraw-Hill.

Leites, Nathan. 1953. A Study of Bolshevism. New York, NY: Free Press. 
Levy, Jack S. 2003. "Political Psychology and Foreign Policy." In Oxford Handbook of Political Psychology, edited by David O. Sears, Leonie Huddy, and Robert Jervis. New York, NY: Oxford University Press.

Lowenthal, Mark M. 1992. "Tribal Tounges: Intelligence Consumer, Intelligence Producers." Washington Quarterly 15.4: 157-168.

Lowenthal, Mark M. 2006. Intelligence: From Secrets to Policy. Washington, DC: CQ Press.

Lijphart, Arend. 1971. "Comparative Politics and the Comparative Method." American Political Science Review 65.3: 682-693.

Luce, Duncan R. and Howard Raiffa. 1957. Games and Decision. New York, NY: John Wiley and Sons.

Macartney, John. 1988. "Intelligence: A Consumer's Guide." Paper presented at the Annual Meeting of the American Political Science Association, Washington, DC: The Washington Hilton.

Malici, Akan. 2005. "Discord and Collaboration Between Allies: Managing External Threats and Internal Cohesion During the 9/11 Era." Journal of Conflict Resolution 49: 90-119.

Malici, Akan. 2006. "Reagan and Gorbachev: Altercasting at the End of the Cold War." In Beliefs and Leadership in World Politics: Methods and Applications of Operational Code Analysis, edited by Mark Schafer and Stephen G. Walker. New York, NY: Palgrave Macmillan.

Malici, Akan and Johnna Malici. 2005. "The Operational Code of Fidel Castro and Kim-Il Sung: The Last Cold Warriors?” Political Psychology 26.3: 387-412.

Marfleet, B. Gregory. 2000. "The Operational Code of John F. Kennedy During the Cuban Missile Crisis: A Comparison of Public and Private Rhetoric." Political Psychology 21.3: 545-558.

Marfleey, Gregory B. and Collen Miller. 2005. 'Failure after 1441: Bush and Chirac in the UN Security Council?” Foreign Policy Analysis 1: 333-359.

Marrin, Stephen. 2006. "Preventing Intelligence Failures by Learning from the Past." International Journal of Intelligence and Counterintelligence 17.4: 655-672.

Maruyama, Magoroh. 1963. "The Second Cybernetics: Deviation-Amplifying Mutual Causal Processes." American Scientist 51: 164-179.

May, Ernest R. 2005. “The Twenty-First Century Challenge for U.S. Intelligence.” In Transforming U.S. Intelligence, edited by Jennifer E. Sims and Burton Gerber. Washington, DC: Georgetown University Press. 
McClellan, David C. 1971. "The Operational Code Approach to the Study of Political Leaders: Dean Acheson's Philosophical and Instrumental Beliefs." Canadian Journal of Political Science 4: 52-75.

McClellan, David C. 1975. Power: The Inner Experience. New York, NY: Irvington Publishers.

Miller, Gerald and Marcia Lynn Whicker. 1998. Handbook of Research Methods in Political Science Lincoln, NE: CRC Press.

Nisbett, Richard E. and David L. Ross. 1980. Human Inference: Strategies and Shortcomings of Social Judgment. Englewood Cliffs, NJ: Prentice-Hall.

Nuendodorf, Kimberly A. 2002. The Content Analysis Guidebook. Thousand Oaks, CA: Sage Publications.

Nuestadt, Robert E. 1970. Alliance Politics. New York, NY: Columbia University Press.

Onuf, Nicholas. 1989. World of Our Making. Columbia, SC: University of South Carolina Press.

Ornstein, Michael D. 1998. “Survey Research.” Current Sociology 46.4: iii-136.

Osgood, Charles E., Sol Saporta, and Jim C. Nunnally. 1956. "Evaluation Assertion Analysis." Litera 3: 47-102.

Phares, Jerry. 1976. Locus of Control in Personality. Morristown, NJ: General Learning Press.

Phythian, Mark. 2006. "The Perfect Intelligence Failure? U.S. Pre-War Intelligence on Iraqi Weapons of Mass Destruction." Politics \& Policy 34.2: 400-424.

Picucci, Peter M. 2008. Terrorism's Operational Code: An Examination of the Belief Systems of Al-Qaeda and Hamas. Ph.D. Dissertation. Lawrence, KS: University of Kansas.

Pickett, George. 1985. "Congress, the Budget and Intelligence." In Intelligence Policy \& Process, edited by Alfred C. Maurer, Marion D. Tunstall, and James M. Keagle. Boulder, CO: Westview Press.

Posner, Richard. 2005. Remaking Domestic Intelligence. Stanford, CA: Hoover Institution Press.

Post, Jerrold M. 2005. The Psychological Assessment of Political Leaders. Ann Arbor, MI: The University of Michigan Press.

Powers, Thomas. 2002. Intelligence Wars: American's Secret History from Hitler to Al Qaeda. New York, NY: New York Review of Books. 
Przeworski, Adam. 1985. "Marxism and Rational Choice." Politics and Society 14: 379-409.

Rhodes, Edward. 1994. "Do Bureaucratic Politics Matter? Some Disconfirming Findings from the Case of the U.S. Navy." World Politics 47.1: 1-41.

Richelson, Jeffrey T. 2007. The US Intelligence Community. Boulder, CO: Westview Press.

Roberts, Fred. 1976. "Strategy for the Energy Crisis: The Case of Commuter Transportation Policy." In Structure of Decision, edited by Robert Axelrod. Princeton, NJ: Princeton University Press.

Robison, Sam. 2006. "George W. Bush and the Vulcans: Leader-Advisor Relations and America's Response to the 9/11 Attacks." In Beliefs and Leadership in World Politics: Methods and Applications of Operational Code Analysis, edited by Mark Schafer and Stephen G. Walker. New York, NY: Palgrave Macmillan.

Rosati, Jerel A. 1981. "Developing a Decision-Making Framework: Bureaucratic Politics in Perspective." World Politics 34: 234-252.

Russell, Kevin. 2004. "The Subjectivity of Intelligence Analysis and Implications for the U.S. National Security Strategy.” SAIS Review 24.1: 147-163.

Schafer, Mark. 2000. "Issues in Assessing Psychological Characteristics: An Introduction to the Symposium." Political Psychology 21.3: 511-527.

Schafer, Mark and Scott Crichlow. 2000. "Bill Clinton's Operational Code: Assessing Source Material Bias." Political Psychology 21.3: 559-571.

Schafer, Mark and Stephen G. Walker. 2001. "Political Leadership and the Democratic Peace: The Operational Code of Prime Minister Tony Blair." In Profiling Political Leaders and the Analysis of Political Leadership, edited by Ofer Feldman and Linda Valenty. Westport, CT: Praeger.

Schafer, Mark and Stephen G. Walker 2006a. "Operational Code Analysis at a Distance: The Verbs in Context System of Content Analysis." In Beliefs and Leadership in World Politics: Methods and Applications of Operational Code Analysis, edited by Mark Schafer and Stephen G. Walker. New York, NY: Palgrave Macmillan.

Schafer, Mark and Stephen G. Walker. 2006b. "Democratic Leaders and the Democratic Peace: The Operational Codes of Tony Blair and Bill Clinton." International Studies Quarterly 50.3: 561-583. 
Schafer, Mark, Stephen G. Walker, and Michael D. Young. 2002. "U.S. Presidents as Conflict Managers: The Operational Codes of George H.W. Bush and Bill Clinton." In Political Leadership for the New Century: Lessons from the Study of Personality and Behavior Among American Leaders, edited by Ofer Feldman and Linda Valenty. Westport, CT: Praeger.

Schein, Edgar H. 1996. "Culture: The Missing Concept in Organization Studies." Administrative Science Quarterly 41.2: 229-240.

Schein, Edgar H. 2004. Organizational Culture and Leadership. New York, NY: John Wiley and Sons.

Schilling, Warner R., Paul Y. Hammond, and Glenn H. Snyder. 1962. Strategy, Politics, and Defense Budgets. New York, NY: Columbia University Press.

Scott, Len and Peter Jackson. 2004. "The Study of Intelligence in Theory and Practice." Intelligence and National Security 19.2: 139-169.

Sears, David O., Leonie Huddy, and Robert Jervis, eds. 2003. Oxford Handbook of Political Psychology. New York, NY: Oxford University Press.

Shapiro, Michael and Matthew Bonham. 1973. "Cognitive Process and Foreign Policy Decision Making." International Studies Quarterly 17: 147-174

Shulsky, Abram N. and Gary J. Schmitt. 1993. Silent Warfare: Understanding the World of Intelligence. Washington, D.C.: Brassey's.

Shulsky, Abram N. and Jennifer Sims. 1992. What is Intelligence? Washington, DC: Consortium for the Study of Intelligence.

Simon, Herbert A. 1957. Models of Man. New York, NY: Wiley.

Sims, Jennifer E. 2005. "Understanding Ourselves." Transforming U.S. Intelligence, edited by Jennifer E. Sims and Burton Gerber. Washington, DC: Georgetown University Press.

Sims, Jennifer E. and Burton Gerber, eds. 2005. Transforming U.S. Intelligence. Washington, DC: Georgetown University Press.

Smith, Michael B. 1968. "A Map for the Analysis of Personality and Politics." Journal of Social Issues 24: 15-28.

Snow, Donald. 1995. National Security: Defense Policy for a New International Order. Boston, MA: Bedford/St. Martins.

Snyder, Mark. 1987. "Public Appearances, Private Realities: The Psychology of Self Monitoring." New York: W.H. Freeman. 
Sprout, Harold and Margaret Sprout. 1965. Man-Milieu Relationship Hypotheses in the Context of International Politics. Princeton, NJ: Princeton University Press.

Stewart, Andrew W. 2006. Friction in U.S. Foreign Policy: Cultural Difficulties with the World. Carlisle, PA: Strategic Studies Institute.

Stempel, John D. 1999. “Error, Foley, and Policy Intelligence." International Journal of Intelligence and Counterintelligence 12.3: 267-281.

Stuart, Douglas and Harvey Starr. 1981. “The Inherent Bad Faith Model Reconsidered: Dulles, Kennedy, and Kissinger." Political Psychology 3: 1-33.

Suedfeld, Peter and A. Dennis Rank. 1976. "Revolutionary Leaders: Long-Term Success as a Function of Changes in Conceptual Complexity." Journal of Personality and Social Psychology 34: 169-178.

Tetlock, Phillip and Antony S.R. Manstead. 1985. "Impression Management versus Intrapsychic Explanations in Social Psychology: A Useful Dichotomy," Psychological Review 92: 59-77.

Tetlock, Phillip. 1998. "Social Psychology and World Politics." In Handbook of Social Psychology, edited by Daniel Gilbert, Susan Fiske, and Gardner Lindsey. New York, NY: McGraw-Hill.

Thies, Cameron G. 2006. "Bankers and Beliefs: The Political Psychology of the Asian Financial Crisis." In Beliefs and Leadership in World Politics: Methods and Applications of Operational Code Analysis, edited by Mark Schafer and Stephen G. Walker. New York, NY: Palgrave Macmillan.

Thomas, Stafford T. 1985. "Intelligence Production and Consumption: A Framework of Analysis." In Intelligence Policy \& Process, edited by Alfred C. Maurer, Marion D. Tunstall, and James M. Keagle. Boulder, CO: Westview Press.

Troy, Thomas F. 1991-1992. “The 'Correct' Definition of Intelligence.” International Journal of Intelligence and Connterintelligence 4: 433-454.

Tolman, Edward C. 1948. "Cognitive Maps in Rats and Man.” Psychological Review 55: 189208.

Turner, Michael A. 1995. "CIA-FBI Non-Cooperation: Cultural Trait or Bureaucratic Inertia? International Journal of Intelligence and Counterintelligence 8.3: 259-273.

Turner, Michael A. 2004. "A Distinctive U.S. Intelligence Identity." International Journal of Intelligence and Counterintelligence 17: 42-61. 
Turner, Michael A. 2005. Historical Dictionary of United States Intelligence. Lanham, MD: The Scarecrow Press, Inc.

Turner, Michael A. 2006. Why Secret Intelligence Fails. Dulles, VA: Potomac Books.

Turner, Stansfield. 1985. Secrecy and Democracy: The CIA in Transition. Boston, MA: Houghton Mills.

Vertzberger, Yaacoc. 1990. The World in Their Minds: Information Processing, Cognition, and Perception in Foreign Policy Decision Making. Stanford, CA: Stanford University Press.

Vital, David. 2003. "Images of Other Peoples in the Making of Intelligence and Foreign Policy." International Journal of Intelligence and Counterintelligence. 16: 16- 33.

Walker, Stephen G. 1977. "The Interface Between Beliefs and Behavior: Henry Kissinger's Operational Code and the Vietnam War." Journal of Conflict Resolution 11: 129-168.

Walker, Stephen G. 1983. "The Motivational Foundations of Political Belief Systems: A Re-Analysis of the Operational Code Construct.” International Studies Quarterly 27: $179-201$.

Walker, Stephen G. 1990. "The Evolution of Operational Code Analysis." Political Psychology 11: 403-418.

Walker, Stephen G. 2004. "Role Identities and the Operational Code of Political Leaders." In Political Psychology as a Perspective on Politics, edited by Margaret Hermann. London, England, UK: Elsevier.

Walker, Stephen G. and Lawrence Falkowski. 1984. "The Operational Codes of U.S. Presidents and Secretaries of State: Motivational Foundations and Behavioral Consequences." Political Psychology 5: 237-266.

Walker, Stephen G. and Mark Schafer. 2000. "The Political Universe of Lyndon B. Johnson and His Advisors: Diagnostic and Strategic Propensities in Their Operational Codes.” Political Psychology 21.3: 529-543.

Walker, Stephen G., Mark Schafer, and Michael D. Young. 1998. "Systematic Procedures for Operational Code Analysis: Measuring and Modeling Jimmy Carter's Operational Code." International Studies Quarterly 42: 175-190.

Walker, Stephen G., Mark Schafer, and Michael D. Young. 1999. "Presidential Operational Codes and Foreign Policy Conflicts in the Post-Cold War World." The Journal of Conflict Resolution 43.5: 610-625.

Wallace, Michael D. and Peter Suedfeld. 1988. "Leadership Performance in Crisis: The Longevity-Complexity Link." International Studies Quarterly 32: 439-451. 
Wark, David L. 1964. "The Definition of Some Estimative Impressions." Studies in Intelligence 8.4: 67-80.

Wark, Wesley K. 2003. "Introduction: Learning to Live with Intelligence." Intelligence and National Security 18.1: 1-14.

Warner, Michael. 2002. "Wanted: A Definition of Intelligence." Studies in Intelligence 46.3: http://www.cia.gov/csi/studies/vol46no3/article02.html.

Watson, George and Dickinson McGraw. 1998. Statistical Inquiry. New York, NY: John Wiley and Sons.

Wendt, Alexander. 1987. "The Agent Structure Problem in International Relations." International Organization 41.3: 335-370.

Wendt, Alexander. 1992. "Anarchy is What States Make of it: The Social Construction of Power Politics.” International Organization 46: 391-425.

Wendt, Alexander. 1994. "Collective Identity Formation and the International State" American Political Science Review 88: 384-396.

Wendt, Alexander. 1995. "Constructing International Politics." International Security 20.1: $71-81$.

Wendt, Alexander. 1999. Social Theory of International Politics. Cambridge, MA: Cambridge University Press.

Winter, David G. 1992. Personality and Foreign Policy: Historical Overview. In Political Psychology and Foreign Policy, edited by Eric Singer and Valerie Hudson. Boulder, CO: Westview Press.

Winter, David G. 2003. "Personality and Political Behavior." In Oxford Handbook of Political Psychology, edited by David O. Sears, Leonie Huddy, and Robert Jervis. New York, NY: Oxford University Press.

Wirtz, James D. 1991. "Intelligence to Please? The Order of Battle Controversy During the Vietnam War.” Political Science Quarterly 106.2: 239-263.

Young, Michael D. 1996. "Cognitive Mapping Meets Semantic Network.” Journal of Conflict Resolution 40: 395-414.

Young, Michael D. and Mark Schafer. 1998. "Is There Method in Our Madness? Ways of Assessing Cognition in International Relations." Mershon International Studies Review 42.1: 63-96. 
Yin, Robert K. 2002. Applications of Case Study Research. Beverly Hills, CA: Sage Publications:

Zegart, Amy. 1999. Flawed By Design: The Evolution of the CIA, JCS, and NSC. Stanford, CA: Stanford University Press.

Ziglio, Erio. 2002. "The Delphi Method and its Contribution to Decision Making. In Gazing Into the Oracle: The Delphic Method and Its Application to Social Policy and Public Health, edited by Michael Adler and Erio Ziglio. London, England, UK: Jessica Kingsley Publishers. 


\section{APPENDIX A}

\section{POPULATION OF DECLASSIFIED NIEs (1950-2007)}

1. Turkey's Position in the East-West Struggle (February 1951)

2. Iran's Position in the East-West Conflict (April 1951)

3. Probable Developments in the World Situation through mid-1953 (October 1951)

4. The Probable Future Orientation of Japan (May 1952)

5. Estimate of the World Situation Through 1955 (October 1953)

6. Communist Courses of Action in Asia Through 1957 (November 1954)

7. Soviet Foreign Policy in the Light of the Summitt Conference (November 1955)

8. Soviet Short-Term Intentions Regarding Berlin and Germany (April 1961)

9. Sino-Soviet Policy and its Probable Effects in Underdeveloped Areas (April 1956)

10. Trends in Soviet Foreign Policy (May 1962)

11. Trends in Soviet Policy toward Sub-Saharan Africa (December 1962)

12. Indonesia's International Orientation (April 1963)

13. The Soviet Role in the Arab World (April 1963)

14. Main Trends in Soviet Foreign Policy (May 1963)

15. Soviet Foreign Policy (February 1964)

16. Prospects for the International Communist Movement (June 1964)

17. Prospects for West German Foreign Policy (April 1965)

18. The Outlook for Sino-Soviet Relations (December 1966)

19. Soviet Foreign Policy (September 1967)

20. Major Trends in Japan's External Relations (January 1968)

21. North Korean Intentions and Capabilities (February 1968)

22. World Trends and Contingencies Affecting U.S. Interests (June 1968)

23. Panama: Prospects for Relations with U.S. (August 1968)

24. The USSR and China (August 1969)

25. Europe, the U.S., and The USSR (December 1969)

26. Soviet Policies in the Middle East and Mediterranean Area (March 1970)

27. Communist China's International Posture (November 1970)

28. Soviet Policy in Asia (April 1971)

29. The Soviet Role in Latin America (April 1971)

30. Soviet Foreign Policies and the Outlook for Soviet-American Relations (April 1972)

31. The USSR and the Changing Scene in Europe (October 1972)

32. Possible Changes in the Sino-Soviet Relationship (October 1973)

33. Cuba's Changing International Role (October 1975)

34. Soviet Strategic Objectives (January 1977)

35. Mexico Under Jose Lopez Portillo: Problems and Prospects for U.S.-Mexican Relations (December 1977)

36. Dealing with Mexico in the 1980's (September 1980)

37. Soviet Goals and Expectations in the Global Power Arena (July 1981)

38. Cuban Policy Toward Latin America (July 1981)

39. The Soviet Challenge to U.S. Security Interests (August 1982)

40. Andropov's Approach to Key U.S.-Soviet Issues (August 1983) 
41. The Changing Sino-Soviet Relationship (April 1984)

42. South Yemen-USSR: Outlook for the Relationship (April 1984)

43. The USSR and the Third World (September 1984)

44. Soviet Policy in East Asia (September 1984)

45. Soviet Policies in Southern Africa (February 1985)

46. The Changing Sino-Soviet Relationship (June 1985)

47. The Soviet Bloc Role in International Terrorism and Revolutionary Violence (August 1986)

48. Soviet Policy Toward the West: The Gorbachev Problem (April 1989)

49. Brazil: Prospects for Economic Reform and Implications for Relations with the U.S. (August 1990)

50. Iran Under Rafsanjani Seeking a New Role in the World Community? (October 1991)

51. The Kurds: Rising Expectations, Old Frustrations (September 1992)

52. Global Humanitarian Emergencies, 1993-94 (October 1993)

53. Prospects for the Worldwide Development of Ballistic Missile Threats to the Continental U.S. (November 1993)

54. Growing Global Migration and its Implications for the U.S. (March 2001)

55. Foreign Missile Developments and the Ballistic Missile Threat Through 2015 (December 2001)

56. Iraq's Continuing Program for Weapons of Mass Destruction (October 2002)

57. Prospects for Iraq's Stability: A Challenging Road Ahead (January 2007)

58. Prospects for Iraq's Stability: Some Progress but Political Reconciliation Elusive (August 2007)

59. Iran: Nuclear Intentions and Capabilities (November 2007) 


\section{APPENDIX B \\ SAMPLING FRAME OF DECLASSIFIED NIEs (1950-2007)}

Subgroup \#1: Cold War I (December 1950-April 1971)

1. Turkey's Position in the East-West Struggle (February 1951)

2. Communist Courses of Action in Asia Through 1957 (November 1954)

3. Sino-Soviet Policy and its Probable Effects in Underdeveloped Areas (April 1956)

4. Indonesia's International Orientation (April 1963)

5. World Trends and Contingencies Affecting U.S. Interests (June 1968)

Subgroup \#2: Détente (May 1971-November 1979)

6. Soviet Foreign Policies and the Outlook for Soviet-American Relations (April 1972)

7. Possible Changes in the Sino-Soviet Relationship (October 1973)

8. Cuba's Changing International Role (October 1975)

9. Soviet Strategic Objectives (January 1977)

10. Mexico Under Jose Lopez Portillo: Problems and Prospects for U.S.-Mexican Relations (December 1977)

Subgroup \#3: Cold War II (December 1979-February 1985)

11. Cuban Policy Toward Latin America (July 1981)

12. The Soviet Challenge to U.S. Security Interests (August 1982)

13. Andropov's Approach to Key U.S.-Soviet Issues (August 1983)

14. Soviet Policy in East Asia (September 1984)

15. The USSR and the Third World (September 1984)

Subgroup \#4: End of Cold War (March 1985-December 1991)

16. The Changing Sino-Soviet Relationship (June 1985)

17. Soviet Policies in Southern Africa (February 1985)

18. The Soviet Bloc Role in International Terrorism and Revolutionary Violence (August 1986)

19. Soviet Policy Toward the West: The Gorbachev Problem (April 1989)

20. Iran Under Rafsanjani Seeking a New Role in the World Community? (October 1991)

Subgroup \#5: Post-Cold War (January 1992-September 11, 2001)

21. Saddam Husayn: Likely to Hang On (June 1992)

22. The Kurds: Rising Expectations, Old Frustrations (September 1992)

23. Global Humanitarian Emeregencies, 1993-94 (October 1993)

24. Prospects for the Worldwide Development of Ballistic Missile Threats to the Continental U.S. (November 1993)

25. Growing Global Migration and its Implications for the U.S. (March 2001) 
Subgroup \#6: Post-9/11 (September 12, 2001-December 2007)

26. Foreign Missile Developments and the Ballistic Missile Threat Through 2015 (December 2001)

27. Iraq's Continuing Program for Weapons of Mass Destruction (October 2002)

28. Prospects for Iraq's Stability: A Challenging Road Ahead (January 2007)

29. Prospects for Iraq's Stability: Some Progress but Political Reconciliation Elusive (August 2007)

30. Iran: Nuclear Intentions and Capabilities (November 2007) 